\title{
GEOMETRY OF SUBELLIPTIC DIFFUSIONS
}

\author{
ANTON THALMAIER
}

\begin{abstract}
These lectures focus on some probabilistic aspects related to subRiemannian geometry. The main intention is to give an introduction to hypoelliptic and subelliptic diffusions. The notes are written from a geometric point of view trying to minimize the weight of "probabilistic baggage" necessary to follow the arguments. We discuss in particular the following topics: stochastic flows to second order differential operators; smoothness of transition probabilities under Hörmander's brackets condition; control theory and Stroock-Varadhan's support theorems; Malliavin calculus; Hörmander's theorem. The notes start from well-known facts in Geometric Stochastic Analysis and guide to recent on-going research topics, like hypoelliptic heat kernel estimates; gradient estimates and Harnack type inequalities for subelliptic diffusion semigroups; notions of curvature related to sub-Riemannian diffusions.
\end{abstract}

\section{Contents}

1. Stochastic flows

1.1. Flow of a vector field

1.2. Flow to a second order differential operator

1.3. What are $L$-diffusions good for?

1.4. $\Gamma$-operators and quadratic variation

2. Construction of stochastic flows

1. Stochastic differential equations on Euclidean space

Stratonovich differentials

2.3. Stochastic differential equations on manifolds

3. Some probabilistic formulas for solutions of PDEs

3.1. Feynman-Kac formula

3.2. Elliptic boundary value problems

3.3. Parabolic boundary value problems 30

4. Stochastic calculus on manifolds 30

4.1. Quadratic variation and integration of 1-forms 30

4.2. Martingales and Brownian motions 34

4.3. Parallel transport and stochastically moving frames 37

4.4. Subelliptic diffusions and sub-Riemannian Brownian motions 45

5. Control theory and support theorems 48

5.1. Control systems 48

5.2. Support theorems $\quad 49$

6. Stochastic flows of diffeomorphisms 50

6.1. Tangent flows and pullback of vector fields under stochastic flows 51

6.2. Malliavin's covariance matrix 52

7. Stochastic flows and hypoellipticity 53

7.1. Hypoellipticity under Hörmander conditions 53

7.2. Girsanov's theorem 58

7.3. Elementary stochastic calculus of variations $\quad 58$

8. Future prospects 60

References

Date: August 2, 2016 File: IHP-Thalmaier.tex. 
In the same way as a vector field on a differentiable manifold induces a flow, second order differential operators induce stochastic flows with similar properties. In this sense, Brownian motion on a Riemannian manifold appears as the stochastic flow associated to the Laplace-Beltrami operator. The new feature of stochastic flows is that the flow curves depend on a random parameter and behave irregularly as functions of time [36]. This irregularity reveals an irreversibility of time which is inherent to stochastic phenomena.

Subelliptic diffusions are stochastic flows to canonical second order differential operators associated with sub-Riemannian structures and corresponding horizontal distributions. A common feature of these operators is their lack of ellipticity. Typically they degenerate along a subbundle of the tangent bundle.

\section{Stochastic Flows}

Let $M$ be a differentiable manifold of dimension $n$ and denote by

$$
T M \stackrel{\pi}{\rightarrow} M
$$

its tangent bundle. In particular, we have

$$
T M=\dot{\cup}_{x \in M} T_{x} M, \quad \pi \mid T_{x} M=x .
$$

The space of smooth sections of $T M$ is denoted by

$$
\begin{aligned}
\Gamma(T M) & =\left\{A: M \rightarrow T M \text { smooth } \mid \pi \circ A=\operatorname{id}_{M}\right\} \\
& =\left\{A: M \rightarrow T M \text { smooth } \mid A(x) \in T_{x} M \text { for all } x \in M\right\}
\end{aligned}
$$

and constitutes the vector fields on $M$. As usual, we identify vector fields on $M$ and $\mathbb{R}$-derivations on $C^{\infty}(M)$ as follows:

$\Gamma(T M) \widehat{=}\left\{A: C^{\infty}(M) \rightarrow C^{\infty}(M) \mathbb{R}\right.$-linear $\left.\mid A(f g)=f A(g)+g A(f) \forall f, g \in C^{\infty}(M)\right\}$

where a vector field $A \in \Gamma(T M)$ is considered as $\mathbb{R}$-derivation via

$$
A(f)(x):=d f_{x} A(x) \in \mathbb{R}, \quad x \in M,
$$

using the differential $d f_{x}: T_{x} M \rightarrow \mathbb{R}$ of $f$ at $x$.

There is a dynamical point of view to vector fields on manifolds: it associates to each vector field a dynamical system given by the flow of the vector field.

1.1. Flow of a vector field. Given a vector field $A \in \Gamma(T M)$. For each $x \in M$ we consider the smooth curve $t \mapsto x(t)$ in $M$ with the properties

$$
x(0)=x \text { and } \dot{x}(t)=A(x(t)) .
$$

We write $\phi_{t}(x):=x(t)$. In this way, we obtain for each $A \in \Gamma(T M)$ the corresponding flow to $A$ given by

$$
\left\{\begin{array}{l}
\frac{d}{d t} \phi_{t}=A\left(\phi_{t}\right), \\
\phi_{0}=\operatorname{id}_{M} .
\end{array}\right.
$$

System (1.2) means that for any $f \in C_{c}^{\infty}(M)$ (space of compactly supported smooth functions on $M$ ) the following conditions hold:

$$
\left\{\begin{array}{l}
\frac{d}{d t}\left(f \circ \phi_{t}\right)=A(f) \circ \phi_{t} \\
f \circ \phi_{0}=f .
\end{array}\right.
$$

Indeed, by the chain rule along with definition (1.1), we have for each $f \in C_{c}^{\infty}(M)$,

$$
\frac{d}{d t}\left(f \circ \phi_{t}\right)=(d f)_{\phi_{t}} \frac{d}{d t} \phi_{t}=(d f)_{\phi_{t}} A\left(\phi_{t}\right)=A(f)\left(\phi_{t}\right) .
$$


In integrated form, for each $f \in C_{c}^{\infty}(M)$, the conditions (1.3) write as:

$$
f \circ \phi_{t}(x)-f(x)-\int_{0}^{t} A(f)\left(\phi_{s}(x)\right) d s=0, \quad t \geq 0, x \in M .
$$

As usual, the curve

$$
\phi .(x): t \mapsto \phi_{t}(x)
$$

is called flow curve (or integral curve) to $A$ starting at $x$.

Remark 1.1. Defining $P_{t} f:=f \circ \phi_{t}$, we observe that $\frac{d}{d t} P_{t} f=P_{t}(A(f))$, in particular

$$
\left.\frac{d}{d t}\right|_{t=0} P_{t} f=A(f)
$$

In other words, from the knowledge of the flow $\phi_{t}$, the underlying vector field $A$ can be recovered by taking the derivative at zero as in Eq. (1.5).

1.2. Flow to a second order differential operator. Now let $L$ be a second order partial differential operator (PDO) on $M$, e.g. of the form

$$
L=A_{0}+\sum_{i=1}^{r} A_{i}^{2}
$$

where $A_{0}, A_{1}, \ldots, A_{r} \in \Gamma(T M)$ for some $r \in \mathbb{N}$. Note that $A_{i}^{2}=A_{i} \circ A_{i}$ is understood as composition of derivations, i.e.

$$
A_{i}^{2}(f)=A_{i}\left(A_{i}(f)\right), \quad f \in C^{\infty}(M) .
$$

Example 1.2. Let $M=\mathbb{R}^{n}$ and consider

$$
A_{0}=0 \text { and } A_{i}=\frac{\partial}{\partial x_{i}} \text { for } i=1, \ldots, n .
$$

Then $L=\Delta$ is the classical Laplace operator on $\mathbb{R}^{n}$.

Alternatively, we may consider partial differentiable operators $L$ on $M$ which locally in a chart $(h, U)$ can be written as

$$
L \mid U=\sum_{i=1}^{n} b_{i} \partial_{i}+\sum_{i, j=1}^{n} a_{i j} \partial_{i} \partial_{j}
$$

where $b \in C^{\infty}\left(U, \mathbb{R}^{n}\right)$ and $a \in C^{\infty}\left(U, \mathbb{R}^{n} \otimes \mathbb{R}^{n}\right)$ such that $a_{i j}=a_{j i}$ for all $i, j$ ( $a$ symmetric). Here we use the notation $\partial_{i}=\frac{\partial}{\partial h_{i}}$.

Motivated by the example of a flow to a vector field (vector fields can be seen as first order differential operators) we want to investigate the question whether an analogous concept of flow exists for second order PDOs.

Question. Is there a notion of a flow to $L$ if $L$ is a second order PDO given by (1.6) or (1.7)?

Definition 1.3. Let $\left(\Omega, \mathscr{F}, \mathbb{P} ;\left(\mathscr{F}_{t}\right)_{t \geq 0}\right)$ be a filtered probability space, i.e. a probability space equipped with increasing sequence of sub- $\sigma$-algebras $\mathscr{F}_{t}$ of $\mathscr{F}$. An adapted continuous process

$$
X .(x) \hat{=}\left(X_{t}(x)\right)_{t \geq 0}
$$

on $\left(\Omega, \mathscr{F}, \mathbb{P} ;\left(\mathscr{F}_{t}\right)_{t \geq 0}\right)$ taking values in $M$, is called flow process to $L$ (or $L$-diffusion) with starting point $x$ if $X_{0}(x)=x$ and if, for all test functions $f \in C_{c}^{\infty}(M)$, the process

$$
N_{t}^{f}(x):=f\left(X_{t}(x)\right)-f(x)-\int_{0}^{t}(L f)\left(X_{s}(x)\right) d s, \quad t \geq 0,
$$


is a martingale, i.e.

$$
\mathbb{E}^{\mathscr{F}_{s}} \underbrace{\left[f\left(X_{t}(x)\right)-f\left(X_{s}(x)\right)-\int_{s}^{t}(L f)\left(X_{r}(x)\right) d r\right]}_{=N_{t}^{f}(x)-N_{s}^{f}(x)}=0, \quad \text { for all } s \leq t .
$$

Note that, by definition, flow processes to a second order PDO depend on an additional random parameter $\omega \in \Omega$. For each $t \geq 0, X_{t}(x) \equiv\left(X_{t}(x, \omega)\right)_{\omega \in \Omega}$ is an $\mathscr{F}_{t}$-measurable random variable. The defining equation (1.4) for flow curves translates to the martingale property of (1.8), i.e. the flow curve condition (1.4) only holds under conditional expectations. The theory of martingales gives a rigorous meaning to the idea of a process without systematic drift [59].

Remark 1.4. Since $N_{0}^{f}(x)=0$, we get from the martingale property of $N^{f}(x)$ that

$$
\mathbb{E}\left[N_{t}^{f}(x)\right]=\mathbb{E}\left[N_{0}^{f}(x)\right]=0 .
$$

Hence, defining $P_{t} f(x):=\mathbb{E}\left[f\left(X_{t}(x)\right)\right]$, we observe that

$$
P_{t} f(x)=f(x)+\int_{0}^{t} \mathbb{E}\left[(L f)\left(X_{s}(x)\right)\right] d s,
$$

and thus

$$
\frac{d}{d t} P_{t} f(x)=\mathbb{E}\left[(L f)\left(X_{t}(x)\right)\right]=P_{t}(L f)(x)
$$

in particular

$$
\left.\left.\frac{d}{d t}\right|_{t=0} \mathbb{E}\left[f\left(X_{t}(x)\right)\right] \equiv \frac{d}{d t}\right|_{t=0} P_{t} f(x)=L f(x) .
$$

The last formula shows that as for deterministic flows we can recover the operator $L$ from its stochastic flow process. To this end however, we have to average over all possible trajectories starting from $x$.

For background on stochastic flows we refer to the monograph of Kunita [36].

Example 1.5 (Brownian motion). Let $M=\mathbb{R}^{n}$ and $L=\frac{1}{2} \Delta$ where $\Delta$ is the Laplacian on $\mathbb{R}^{n}$. Let $X \equiv\left(X_{t}\right)$ be a Brownian motion on $\mathbb{R}^{n}$ starting at the origin. By Itô's formula [52], for $f \in C^{\infty}\left(\mathbb{R}^{n}\right)$, we have

$$
\begin{aligned}
d\left(f \circ X_{t}\right) & =\sum_{i=1}^{n} \partial_{i} f\left(X_{t}\right) d X_{t}^{i}+\frac{1}{2} \sum_{i, j=1}^{n} \partial_{i} \partial_{j} f\left(X_{t}\right) d X_{t}^{i} d X_{t}^{j} \\
& =\left\langle(\nabla f)\left(X_{t}\right), d X_{t}\right\rangle+\frac{1}{2}(\Delta f)\left(X_{t}\right) d t .
\end{aligned}
$$

Thus, for each $f \in C_{c}^{\infty}\left(\mathbb{R}^{n}\right)$,

$$
f\left(X_{t}\right)-f\left(X_{0}\right)-\int_{0}^{t} \frac{1}{2}(\Delta f)\left(X_{s}\right) d s, \quad t \geq 0,
$$

is a martingale. This means that the process

$$
X_{t}(x):=x+X_{t}
$$

is an $L$-diffusion to $\frac{1}{2} \Delta$ in the sense of Definition 1.3.

Remarks 1.6. As for deterministic flows, we have to deal with the problem that stochastic flows may explode in finite times. 
1. We allow $X .(x)$ to be defined only up to some stopping time $\zeta(x)$, i.e.

$$
X .(x) \mid[0, \zeta(x)[
$$

where

$$
\{\zeta(x)<\infty\} \subset\left\{\lim _{t \uparrow \zeta(x)} X_{t}(\omega)=\infty \text { in } \hat{M}:=M \dot{\cup}\{\infty\}\right\} \mathbb{P} \text {-a.s. }
$$

Here $\hat{M}$ denotes the one-point-compactification of $M$. A stopping time $\zeta(x)$ with property (1.9) is called (maximal) lifetime for the process $X .(x)$ starting at $x$. In equivalent terms, let $U_{n} \subset M$ be open, relatively compact subsets exhausting $M$ in the sense that

$$
U_{n} \subset \bar{U}_{n} \subset U_{n+1} \subset \ldots, \quad \bar{U}_{n} \text { compact, and } \cup_{n} U_{n}=M .
$$

Then we have $\zeta(x)=\sup _{n} \tau_{n}(x)$ for the maximal lifetime of $X$. $(x)$ where $\tau_{n}(x)$ is the family of stopping times (first exit times of $U_{n}$ ) defined by

$$
\tau_{n}(x):=\inf \left\{t \geq 0: X_{t}(x) \notin U_{n}\right\} .
$$

2. For $f \in C^{\infty}(M)$ (not necessarily compactly supported), the process $N^{f}(x)$ will in general only be a local martingale [52], i.e. there exist stopping times $\tau_{n} \uparrow \zeta(x)$ such that

$$
\forall n \in \mathbb{N}, \quad\left(N_{t \wedge \tau_{n}}^{f}(x)\right)_{t \geq 0} \text { is a (true) martingale. }
$$

3. The following two statements are equivalent (the proof will be given later):

(a) The process

$$
f(X .(x))=\left(f\left(X_{t}(x)\right)\right)_{t \geq 0}
$$

is of locally bounded variation for all $f \in C_{c}^{\infty}(M)$.

(b) The operator $L$ is of first order, i.e. $L$ is a vector field (in which case the flow is deterministic).

In other words, flow processes have "nice paths" (for instance, paths of bounded variation) if and only if the corresponding operator is first order (i.e. a vector field).

1.3. What are $L$-diffusions good for? Before discussing the problem of how to construct $L$-diffusions, we want to study some implications to indicate the usefulness and power of this concept. In the following two examples we only assume existence of an $L$-diffusion to a given operator $L$.

A. (Dirichlet problem) Let $\varnothing \neq D \subsetneq M$ be an open, connected, relatively compact domain, $\varphi \in C(\partial D)$ and let $L$ be a second order PDO on $M$. The Dirichlet problem (DP) is the problem to find a function $u \in C(\bar{D}) \cap C^{2}(D)$ such that

$$
\left\{\begin{array}{l}
L u=0 \text { on } D \\
\left.u\right|_{\partial D}=\varphi
\end{array}\right.
$$

Suppose that there is an $L$-diffusion $\left(X_{t}(x)\right)_{t>0}$. We choose a sequence of open domains $D_{n} \uparrow D$ such that $\bar{D}_{n} \subset D$, and for each $n$, we consider the first exit time of $D_{n}$,

$$
\tau_{n}(x)=\inf \left\{t \geq 0, X_{t}(x) \notin D_{n}\right\} .
$$

Then $\tau_{n}(x) \uparrow \tau(x)$ where

$$
\tau(x)=\sup _{n} \tau_{n}(x)=\inf \left\{t \geq 0, X_{t}(x) \notin D\right\} .
$$

Now assume that $u$ is a solution to (DP). We may choose test functions $u_{n} \in$ $C_{c}^{\infty}(M)$ such that $u_{n}\left|D_{n}=u\right| D_{n}$ and supp $u_{n} \subset D$. Then, by the property of an $L$-diffusion,

$$
N_{t}(x):=u_{n}\left(X_{t}(x)\right)-u_{n}(x)-\int_{0}^{t}\left(L u_{n}\right)\left(X_{r}(x)\right) d r
$$


is a martingale. We suppose that $x \in D_{n}$. Then

$$
\begin{aligned}
N_{t \wedge \tau_{n}(x)}(x) & =u_{n}\left(X_{t \wedge \tau_{n}(x)}(x)\right)-u_{n}(x)-\int_{0}^{t \wedge \tau_{n}(x)} \underbrace{\left(L u_{n}\right)\left(X_{r}(x)\right)}_{=0} d r \\
& =u\left(X_{t \wedge \tau_{n}(x)}(x)\right)-u(x)
\end{aligned}
$$

is also a martingale (here we used that the integral in (1.10) is zero since $L u_{n}=$ $L u=0$ on $\left.D_{n}\right)$. Thus we get

$$
\mathbb{E}\left[N_{t \wedge \tau_{n}(x)}(x)\right]=\mathbb{E}\left[N_{0}(x)\right]=0
$$

which shows that for each $n \in \mathbb{N}$,

$$
u(x)=\mathbb{E}\left[u\left(X_{t \wedge \tau_{n}(x)}(x)\right)\right] .
$$

From Eq. (1.11) we may conclude by dominated convergence and since $\tau_{n}(x) \uparrow \tau$ that

$$
u(x)=\lim _{n \rightarrow \infty} \mathbb{E}\left[u\left(X_{t \wedge \tau_{n}(x)}(x)\right)\right]=\mathbb{E}\left[\lim _{n \rightarrow \infty} u\left(X_{t \wedge \tau_{n}(x)}(x)\right)\right]=\mathbb{E}\left[u\left(X_{t \wedge \tau(x)}(x)\right)\right] .
$$

We now make the hypothesis that $\tau(x)<\infty$ a.s. (the process exits the domain $D$ in finite time). Then

$$
\begin{aligned}
u(x) & =\lim _{t \rightarrow \infty} \mathbb{E}\left[u\left(X_{t \wedge \tau(x)}(x)\right)\right]=\mathbb{E}\left[\lim _{t \rightarrow \infty} u\left(X_{t \wedge \tau(x)}(x)\right)\right] \\
& =\mathbb{E}\left[u\left(X_{\tau(x)}(x)\right)\right]=\mathbb{E}\left[\varphi\left(X_{\tau(x)}(x)\right)\right]
\end{aligned}
$$

where for the last equality we used the boundary condition $u \mid \partial D=\varphi$. Note that by passing to the image measure $\mu_{x}:=\mathbb{P} \circ X_{\tau(x)}(x)^{-1}$ on the boundary we get

$$
\mathbb{E}\left[\varphi\left(X_{\tau(x)}(x)\right)\right]=\int_{\partial D} \varphi(z) \mu_{x}(d z) .
$$

Notation 1.7. The measure $\mu_{x}$, defined on Borel sets $A \subset \partial D$,

$$
\mu_{x}(A)=\mathbb{P}\left\{X_{\tau(x)}(x) \in A\right\},
$$

is called exit measure from the domain $D$ of the diffusion $X_{t}(x)$. It represents the probability that the process $X_{t}$, when started at $x$ in $D$, exits the domain $D$ through the boundary set $A$.

Conclusions. From the discussion of the Dirichlet problem above we can make the following two observations.

(a) (Uniqueness) Under the hypothesis

$$
\tau(x)<\infty \text { a.s. for all } x \in D
$$

we have uniqueness of the solutions to the Dirichlet problem (DP). It will be shown later that this hypothesis concerns non-degeneracy of the operator $L$.

(b) (Existence) Under the hypothesis

$$
\tau(x) \rightarrow 0 \text { if } D \ni x \rightarrow a \in \partial D
$$

we have

$$
\mathbb{E}\left[\varphi\left(X_{\tau(x)}(x)\right)\right] \rightarrow \varphi(a), \quad \text { if } D \ni x \rightarrow a \in \partial D .
$$

Thus one may define $u(x):=\mathbb{E}\left[\varphi\left(X_{\tau(x)}(x)\right)\right]$. It can be shown then that $u$ is $L$-harmonic on $D$ if it is twice differentiable; thus under the hypothesis in (b), $u$ will then satisfy the boundary condition and hence solve (DP). The hypothesis in (b) is obviously a regularity condition on the boundary $\partial D$. 
Note that in the arguments above we nowhere used the explicit form of the operator $L$ nor of the domain $D$. We only used the general properties of a stochastic flow process associated to the given operator $L$. For a more complete discussion of the Dirichlet problem see [54, 13].

\section{Examples 1.8.}

(1) Let $M=\mathbb{R}^{2} \backslash\{0\}$ and $D=\left\{x \in \mathbb{R}^{2}: r_{1}<|x|<r_{2}\right\}$ with $0<r_{1}<r_{2}$. Consider the operator

$$
L=\frac{1}{2} \frac{\partial^{2}}{\partial \vartheta^{2}}
$$

where $\vartheta$ denotes the angle when passing to polar coordinates on $M$. If $u$ is a solution of (DP), then $u+v(r)$ is a solution of (DP) as well, for any radial function $v(r)$ satisfying $v\left(r_{1}\right)=v\left(r_{2}\right)=0$. Hence, uniqueness of solutions fails.

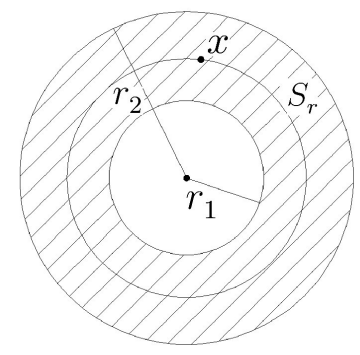

Note: For $x \in D$ with $|x|=r$, let $S_{r}=\left\{x \in \mathbb{R}^{2}:|x|=r\right\}$. Then, the flow process $X .(x)$ to $L$ is easily seen to be a (one-dimensional) Brownian motion on $S_{r}$. In particular,

$$
\tau(x)=+\infty \text { a.s. }
$$

(2) Let $M=\mathbb{R}^{2}$ and consider the operator

$$
L=\frac{1}{2} \frac{\partial^{2}}{\partial x_{1}^{2}}
$$

on a domain $D$ in $\mathbb{R}^{2}$ of the following shape:

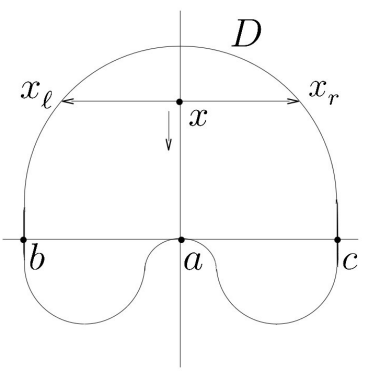

Then, for $x=\left(x_{1}, x_{2}\right) \in D$, the flow process $X$. $(x)$ starting at $x$ is a (onedimensional) Brownian motion on $\mathbb{R} \times\left\{x_{2}\right\}$. In other words, flow processes move on horizontal lines. In particular, when started at $x \in D$, the process can only exit at two points (e.g. $x_{\ell}$ and $x_{r}$ in the picture). Letting $x$ vertically approach $a$, by symmetry of the one-dimensional Brownian motion, 
we see that there exists a solution of (DP) if and only if

$$
\varphi(a)=\frac{\varphi(b)+\varphi(c)}{2} .
$$

B. (Heat equation) Let $L$ be a second order PDO on $M$ and fix $f \in C(M)$. The heat equation on $M$ with initial condition $f$ concerns the problem of finding a real-valued function $u=u(t, x)$ defined on $\mathbb{R}_{+} \times M$ such that

$$
\left\{\begin{array}{l}
\left.\frac{\partial u}{\partial t}=L u \quad \text { on }\right] 0, \infty[\times M, \\
\left.u\right|_{t=0}=f .
\end{array}\right.
$$

Suppose now that there is an $L$-diffusion $X$. $(x)$. It is straightforward to see that the "time-space process" $\left(t, X_{t}(x)\right)$ will then be a $\hat{L}$-diffusion for the parabolic operator

$$
\hat{L}=\frac{\partial}{\partial t}+L
$$

with starting point $(0, x)$. By definition, this means that for all $\varphi \in C^{2}\left(\mathbb{R}_{+} \times M\right)$,

$$
d \varphi\left(t, X_{t}(x)\right)-(\hat{L} \varphi)\left(t, X_{t}(x)\right) d t \stackrel{\mathrm{m}}{=} 0
$$

where $\stackrel{m}{=}$ denotes equality modulo differentials of local martingales.

From now on we assume non-explosion of the $L$-diffusion. In other words, we adopt the hypothesis that $\zeta(x)=+\infty$ a.s. for all $x \in M$, i.e.

$$
\mathbb{P}\left\{X_{t}(x) \in M, \forall t \geq 0\right\}=1, \quad \forall x \in M .
$$

Suppose now that $u$ is a bounded solution of (HE). We fix $t \geq 0$ and consider the restriction $u \mid[0, t] \times M$. Then

$$
u\left(t-s, X_{s}(x)\right)-u(t, x)-\int_{0}^{s}\left[\left(\frac{\partial}{\partial r}+L\right) u(t-r, \cdot)\right]\left(X_{r}(x)\right) d r, \quad 0 \leq s<t,
$$

is a local martingale. In other words, fixing $t>0$, we have for $0 \leq s<t$,

$$
\begin{aligned}
u\left(t-s, X_{s}(x)\right)=u(t, x) & +\int_{0}^{s} \underbrace{\left(\frac{\partial}{\partial r}+L\right) u(t-r, \cdot)}_{=0, \text { since } u \text { solves (HE) }}\left(X_{r}(x)\right) d r \\
& +(\text { local martingale) })_{s} .
\end{aligned}
$$

Since the integral in (1.12) vanishes, we see that the local martingale term in (1.12) is actually a bounded local martingale ( since $u\left(t-s, X_{s}(x)\right)-u(t, x)$ is bounded) and hence a true martingale (equal to zero at time 0 ). Using the martingale property we first take expectations and then pass to the limit as $s \uparrow t$ to obtain

$$
u(t, x)=\mathbb{E}\left[u\left(t-s, X_{s}(x)\right)\right] \rightarrow \mathbb{E}\left[u\left(0, X_{t}(x)\right)\right]=\mathbb{E}\left[f\left(X_{t}(x)\right)\right], \quad \text { as } s \uparrow t,
$$

where for the limit in (1.13) we used dominated convergence (recall that $u$ is bounded).

Conclusion. Under the hypothesis $\zeta(x)=+\infty$ for all $x \in M$, we have uniqueness of (bounded) solutions to the heat equation (HE). Solutions are necessarily of the form

$$
u(t, x)=\mathbb{E}\left[f\left(X_{t}(x)\right)\right]
$$

Interpretation. The solution $u(t, x)$ at time $t$ and at point $x$ can be constructed as follows: run an $L$-diffusion process starting from $x$ up time $t$, apply the initial condition $f$ to the obtained random position $X_{t}(x)$ at time $t$ and average over all possible paths. 


\section{4. $\Gamma$-operators and quadratic variation.}

Definition 1.9. Let $L: C^{\infty}(M) \rightarrow C^{\infty}(M)$ be a linear mapping (for instance a second order PDO). The $\Gamma$-operator associated to L ("l'operateur carré du champ") is the bilinear map

$$
\begin{gathered}
\Gamma: C^{\infty}(M) \times C^{\infty}(M) \rightarrow C^{\infty}(M) \text { given as } \\
\Gamma(f, g):=\frac{1}{2}[L(f g)-f L(g)-g L(f)] .
\end{gathered}
$$

Example 1.10. Let $L$ be a second order PDO on $M$ without constant term (i.e. $L 1=0)$. Suppose that in a local chart $(h, U)$ for $M$ the operator $L$ writes as

$$
L \mid C_{U}^{\infty}(M)=\sum_{i, j=1}^{n} a_{i j} \partial_{i} \partial_{j}+\sum_{i=1}^{n} b_{i} \partial_{i}
$$

where $C_{U}^{\infty}(M)=\left\{f \in C^{\infty}(M): \operatorname{supp} f \subset U\right\}$ and $\partial_{i}=\frac{\partial}{\partial h_{i}}$. Then

$$
\Gamma(f, g)=\sum_{i, j=1}^{n} a_{i j}\left(\partial_{i} f\right)\left(\partial_{j} g\right), \quad \forall f, g \in C_{U}^{\infty}(M) .
$$

For instance, in the special case that $M=\mathbb{R}^{n}$ and $L=\Delta$, we find

$$
\Gamma(f, f)=\|\nabla f\|^{2} .
$$

Remark 1.11. Let $L$ be a second order PDO. Then the following equivalence holds:

$\Gamma(f, g)=0 \quad \forall f, g \in C^{\infty}(M)$ if and only if $L$ is of first order, i.e. $L \in \Gamma(T M)$.

For instance, if $L=A_{0}+\sum_{i=1}^{r} A_{i}^{2}$, then

$$
\Gamma(f, g)=\sum_{i=1}^{r} A_{i}(f) A_{i}(g),
$$

and in particular

$$
\Gamma \equiv 0 \quad \text { if and only if } \quad A_{1}=A_{2}=\ldots=A_{r}=0 .
$$

Remark 1.12. A continuous real-valued stochastic process $\left(X_{t}\right)_{t \geq 0}$ is called a semimartingale if it can be decomposed as

$$
X_{t}=X_{0}+M_{t}+A_{t}
$$

where $M$ is a local martingale and $A$ an adapted process of locally bounded variation (with $M_{0}=A_{0}=0$ ). The representation of a semimartingale $X$ as in (1.14) (Doob-Meyer decomposition) is unique: if $\mathscr{M}_{0}$ denotes the class of local martingales starting from 0 and $\mathscr{A}_{0}$ is the class of adapted process with paths of locally bounded variation starting from 0 , then $\mathscr{M}_{0} \cap \mathscr{A}_{0}=0$.

Definition 1.13. Let $X$ be a continuous adapted process taking values in a manifold $M$. Then $X$ is called semimartingale on $M$ if

$$
f(X) \equiv\left(f\left(X_{t}\right)\right)_{t \geq 0}
$$

is a real-valued semimartingale for all $f \in C^{\infty}(M)$. 
Remark 1.14. If $X$ has maximal lifetime $\zeta$, i.e.,

$$
\{\zeta<\infty\} \subset\left\{\lim _{t \uparrow \zeta} X_{t}=\infty \text { in } \hat{M}=M \dot{\cup}\{\infty\}\right\} \text { a.s. }
$$

then $f(X)$ is well-defined as a process globally on $\mathbb{R}_{+}$for all $f \in C_{c}^{\infty}(M)$ (with the convention $f(\infty)=0)$. For $f \in C^{\infty}(M)$, in general,

$$
f(X) \equiv\left(f\left(X_{t}\right)\right)_{t<\zeta}
$$

is only a semimartingale with lifetime $\zeta$.

Proposition 1.15. Let $L: C^{\infty}(M) \rightarrow C^{\infty}(M)$ be an $\mathbb{R}$-linear map and $X$ be a semimartingale on $M$ such that for all $f \in C^{\infty}(M)$,

$$
N_{t}^{f}:=f\left(X_{t}\right)-f\left(X_{0}\right)-\int_{0}^{t} L f\left(X_{r}\right) d r
$$

is a continuous local martingale (of same lifetime as $X$ ) (i.e. $d(f(X))-L f(X) d t \stackrel{\mathrm{m}}{=}$ 0 where $\stackrel{m}{=}$ denotes equality modulo differentials of local martingales).

Then, for all $f, g \in C^{\infty}(M)$, the quadratic variation $[f(X), g(X)]$ of $f(X)$ and $g(X)$ is given by

$$
d[f(X), g(X)] \equiv d\left[N^{f}, N^{g}\right]=2 \Gamma(f, g)(X) d t .
$$

In particular, $\Gamma(f, f)(X) \geq 0$ a.s.

Proof. Let $f \in C^{\infty}\left(M, \mathbb{R}^{r}\right)$ and $\phi \in C^{\infty}\left(\mathbb{R}^{r}\right)$. Writing as above $\stackrel{m}{=}$ for equality modulo differentials of local martingales, we have

$$
d(\phi \circ f)(X) \stackrel{\mathrm{m}}{=} L(\phi \circ f)(X) d t .
$$

Developing the left-hand side in Eq. (1.15) by Itô's formula (the function $\phi$ is applied to the semimartingale $f \circ X)$, we get

$$
\begin{aligned}
& d(\phi(f(X))) \\
& \quad=\sum_{i=1}^{r}\left(D_{i} \phi\right)(f \circ X) d\left(f^{i} \circ X\right)+\frac{1}{2} \sum_{i, j=1}^{r}\left(D_{i} D_{j} \phi\right)(f \circ X) d\left[f^{i}(X), f^{j}(X)\right] \\
& \quad \stackrel{\mathrm{m}}{=} \sum_{i=1}^{r}\left(D_{i} \phi\right)(f \circ X)\left(L f^{i}\right)(X) d t+\frac{1}{2} \sum_{i, j=1}^{r}\left(D_{i} D_{j} \phi\right)(f \circ X) d\left[f^{i}(X), f^{j}(X)\right]
\end{aligned}
$$

where $D_{i}=\partial / \partial x_{i}$. By equating the drift parts we find

$$
\left[L(\phi \circ f)-\sum_{i=1}^{r}\left(\left(D_{i} \phi\right) \circ f\right)\left(L f^{i}\right)\right](X) d t=\frac{1}{2} \sum_{i, j=1}^{r}\left(D_{i} D_{j} \phi\right)(f \circ X) d\left[f^{i}(X), f^{j}(X)\right] .
$$

Taking now $r=2$ and considering the special case $\phi(x, y)=x y$, we get with $f=\left(f^{1}, f^{2}\right)$,

$$
\left[L\left(f^{1} f^{2}\right)-f^{1} L\left(f^{2}\right)-f^{2} L\left(f^{1}\right)\right](X) d t=d\left[f^{1}(X), f^{2}(X)\right] .
$$

This completes the proof since $\left[L\left(f^{1} f^{2}\right)-f^{1} L\left(f^{2}\right)-f^{2} L\left(f^{1}\right)\right](X)=2 \Gamma\left(f^{1}, f^{2}\right)(X)$.

Lemma 1.16. For an $\mathbb{R}$-linear map $L: C^{\infty}(M) \rightarrow C^{\infty}(M)$ the following statements are equivalent:

(i) L is a second order PDO (without constant term) 
(ii) L satisfies the second order chain rule, i.e. for all $f \in C^{\infty}\left(M, \mathbb{R}^{r}\right)$ and $\phi \in$ $C^{\infty}\left(\mathbb{R}^{r}\right)$,

$$
L(\phi \circ f)=\sum_{i=1}^{r}\left(D_{i} \phi \circ f\right)\left(L f^{i}\right)+\sum_{i, j=1}^{r}\left(D_{i} D_{j} \phi \circ f\right) \Gamma\left(f^{i}, f^{j}\right) .
$$

Proof. (i) $\Rightarrow$ (ii): Write $L$ in local coordinates as

$$
L \mid C_{U}^{\infty}(M)=\sum_{i, j=1}^{n} a_{i j} \partial_{i} \partial_{j}+\sum_{i=1}^{n} b_{i} \partial_{i}
$$

and use that $\Gamma(f, g)=\sum_{i, j=1}^{n} a_{i j} \partial_{i} f \partial_{j} g$.

(ii) $\Rightarrow$ (i): Determine the action of $L$ on functions $\varphi$ written in local coordinates $(h, U)$ via

$$
L(\varphi) \mid U=L\left(\varphi \circ h^{-1} \circ h\right) \equiv L(\phi \circ f)
$$

where $\phi=\varphi \circ h^{-1}$ and $f=h$. Details are left as an exercise to the reader.

Corollary 1.17. Let $L: C^{\infty}(M) \rightarrow C^{\infty}(M)$ be an $\mathbb{R}$-linear mapping. Suppose that for each $x \in M$ there exists a semimartingale $X$ on $M$ such that $X_{0}=x$ and such that for each $f \in C^{\infty}(M)$,

$$
f\left(X_{t}\right)-f(x)-\int_{0}^{t} L f(X) d r
$$

is a local martingale. Then $L$ is necessary a PDO of order at most 2.

In addition, $X$ has "nice" trajectories (e.g. in the sense that $[f(X), f(X)]=0$ for all $f \in C^{\infty}(M)$ ) if and only if $L$ is first order.

Proof. As in the proof of Proposition 1.15, for all $f \in C^{\infty}\left(M, \mathbb{R}^{r}\right)$ and $\phi \in C^{\infty}\left(\mathbb{R}^{r}\right)$, we have

$$
\left[L(\phi \circ f)-\sum_{i=1}^{r}\left(D_{i} \phi \circ f\right)\left(L f^{i}\right)+\sum_{i, j=1}^{r}\left(D_{i} D_{j} \phi \circ f\right) \Gamma\left(f^{i}, f^{j}\right)\right](X)=0,
$$

so that $L$ is a second order PDO by Lemma 1.16. The second claim uses

$$
d[f(X), g(X)]=2 \Gamma(f, g)(X) d t, \quad f, g \in C^{\infty}(M) .
$$

\section{Construction of Stochastic Flows}

Flows to vector fields are classically constructed as solutions of ordinary differential equations on manifolds. In the same way, stochastic flows can be constructed as solutions to stochastic differential equations (SDE) on manifolds. We start by recalling same basic facts about stochastic differential equations on $\mathbb{R}^{n}$.

\subsection{Stochastic differential equations on Euclidean space.}

Example 2.1 (SDE on $\mathbb{R}^{n}$ ). Given $\beta: \mathbb{R}_{+} \times \mathbb{R}^{n} \rightarrow \mathbb{R}^{n}$ and in addition a function

$$
\sigma: \mathbb{R}_{+} \times \mathbb{R}^{n} \rightarrow \operatorname{Hom}\left(\mathbb{R}^{r}, \mathbb{R}^{n}\right) \equiv \operatorname{Matr}(n \times r, \mathbb{R}) .
$$

Let $B$ be a Brownian motion on $\mathbb{R}^{r}$. Now one wants to find a continuous semimartingale $Y$ on $\mathbb{R}^{n}$ such that

$$
d Y_{t}=\beta\left(t, Y_{t}\right) d t+\sigma\left(t, Y_{t}\right) d B_{t}
$$

in the sense of Itô, i.e.

$$
Y_{t}=Y_{0}+\int_{0}^{t} \beta\left(s, Y_{s}\right) d s+\int_{0}^{t} \sigma\left(s, Y_{s}\right) d B_{s} .
$$


In Eq. (2.1) the first term describes the "systematic part" (drift term) in the evolution of $Y$, whereas the second integral represents the "fluctuating part" (diffusion term).

Definition 2.2. An $\mathbb{R}^{n}$-valued stochastic process $\left(Y_{t}\right)_{t \geq 0}$ is called Itô process if it has a representation as

$$
Y_{t}=Y_{0}+\int_{0}^{t} K_{s} d s+\int_{0}^{t} H_{s} d B_{s}
$$

where

- $Y_{0}$ is $\mathscr{F}_{0}$-measurable;

- $K_{s}$ and $H_{s}$ are adapted processes taking values in $\mathbb{R}^{n}$, resp. $\operatorname{Hom}\left(\mathbb{R}^{r}, \mathbb{R}^{n}\right)$;

- $\mathbb{E}\left[\int_{0}^{t}\left|K_{s}\right| d s\right]<\infty$ and $\mathbb{E}\left[\int_{0}^{t} H_{s}^{2} d s\right]<\infty$ for each $t \geq 0$.

Proposition 2.3. Let $\beta$ : $\mathbb{R}_{+} \times \mathbb{R}^{n} \rightarrow \mathbb{R}^{n}$ and $\sigma: \mathbb{R}_{+} \times \mathbb{R}^{n} \rightarrow \operatorname{Hom}\left(\mathbb{R}^{r}, \mathbb{R}^{n}\right)$ be continuous functions. For a continuous semimartingale $Y$ on $\mathbb{R}^{n}$, defined up to some predictable stopping time $\tau$ (i.e. there exists a sequence of stopping times $\tau_{n}<\tau$ with $\tau_{n} \uparrow \tau$ ), the following conditions are equivalent:

(a) $Y$ is a solution of the $\mathrm{SDE}$

$$
d Y_{t}=\beta\left(t, Y_{t}\right) d t+\sigma\left(t, Y_{t}\right) d B_{t} \quad \text { on }[0, \tau[
$$

i.e.,

$$
Y_{t}=Y_{0}+\int_{0}^{t} \beta\left(s, Y_{s}\right) d s+\int_{0}^{t} \sigma\left(s, Y_{s}\right) d B_{s}, \quad \forall 0 \leq t<\tau \text { a.s. }
$$

(b) For all $f \in C^{\infty}\left(\mathbb{R}^{n}\right)$,

$$
d(f \circ Y)=(L f)(t, Y) d t+\sum_{k=1}^{n} \sum_{i=1}^{r} \sigma_{k i}(t, Y) D_{k} f(Y) d B_{i} \quad \text { on }[0, \tau[
$$

where

$$
L=\sum_{k=1}^{n} \beta_{k} D_{k}+\frac{1}{2} \sum_{k, \ell=1}^{n}\left(\sigma \sigma^{*}\right)_{k \ell} D_{k} D_{\ell}
$$

where $\sigma^{*}$ is a transpose of $\sigma$, and $\left(\sigma \sigma^{*}\right)_{k \ell}=\sum_{i=1}^{r} \sigma_{k i} \sigma_{\ell i}$. In particular, every solution of (2.2) is an L-diffusion on $[0, \tau[$ in the sense that

$$
d(f \circ Y)-L f(t, Y) d t=d(\text { local martingale }) \text { on }[0, \tau[\text {. }
$$

Proof. (a) $\Rightarrow$ (b) Let $Y$ be a solution of $\operatorname{SDE}(2.2)$. Then

$$
d Y^{k} d Y^{\ell} \equiv d\left[Y^{k}, Y^{\ell}\right]=\left(\sigma \sigma^{*}\right)_{k \ell}(t, Y) d t
$$

where $\left[Y^{k}, Y^{\ell}\right]$ represents quadratic covariation of $Y^{k}$ and $Y^{\ell}$. By Itô's formula we get

$$
\begin{aligned}
d(f \circ Y)= & \sum_{k=1}^{n} D_{k} f(Y)\left(\beta_{k}(t, Y) d t+\sum_{i=1}^{r} \sigma_{k i}(t, Y) d B^{i}\right) \\
& +\frac{1}{2} \sum_{k, \ell=1}^{n} D_{k} D_{\ell} f(Y) \underbrace{\left(\sigma \sigma^{*}\right)_{k \ell}(t, Y) d t}_{=d\left[Y^{k}, Y^{\ell}\right]} \\
= & L f(t, Y) d t+\sum_{k=1}^{n} \sum_{i=1}^{r} \sigma_{k i}(t, Y) D_{k} f(t, Y) d B_{i} \\
= & L f(t, Y) d t+d(\text { local martingale }) .
\end{aligned}
$$


(b) $\Rightarrow$ (a) Take $f(x)=x_{\ell}$. Then $D_{k} f=\delta_{k \ell}$ and $L f=\beta_{\ell}$, thus

$$
d Y^{\ell}=\beta_{\ell}(t, Y) d t+\sum_{i=1}^{r} \sigma_{\ell i}(t, Y) d B^{i} \quad \text { for each } \ell=1, \ldots, n .
$$

This shows that $Y$ solves $\operatorname{SDE}(2.2)$ on $[0, \tau[$.

Proposition 2.4 (Itô SDE on $\mathbb{R}^{n}$; case of global Lipschitz conditions). Let $Z$ be a continuous semimartingale on $\mathbb{R}^{r}$ and

$$
\alpha: \mathbb{R}^{n} \rightarrow \operatorname{Hom}\left(\mathbb{R}^{r}, \mathbb{R}^{n}\right)(=\operatorname{Matr}(n \times r ; \mathbb{R}))
$$

such that

$$
\exists L>0, \quad|\alpha(y)-\alpha(z)| \leq L|y-z| \quad \forall y, z \in \mathbb{R}^{n} \quad \text { (global Lipschitz conditions). }
$$

Then, for each $\mathscr{F}_{0}$-measurable $\mathbb{R}^{n}$-valued random variable $x_{0}$, there exists a unique continuous semimartingale $\left(X_{t}\right)_{t \geq 0}$ on $\mathbb{R}^{n}$ such that

$$
d X=\alpha(X) d Z \text { and } X_{0}=x_{0} .
$$

Uniqueness holds in the following sense: suppose that $Y$ is another continuous semimartingale such that $d Y=\alpha(Y) d Z$ and $Y_{0}=x_{0}$, then $X_{t}=Y_{t}$ for all $t$ a.s.

Proof. The proof is standard in Stochastic Analysis, see for instance [51] or [30].

Proposition 2.5 (Itô SDEs on $\mathbb{R}^{n}$ : case of the local Lipschitz coefficients). Let $Z$ be a continuous semimartingale on $\mathbb{R}^{r}$ and let

$$
\alpha: \mathbb{R}^{n} \rightarrow \operatorname{Hom}\left(\mathbb{R}^{r}, \mathbb{R}^{n}\right),
$$

be locally Lipschitz, i.e. for each compact $K \subset \mathbb{R}^{n}$ there exists a constant $L_{K}>0$ such that

$$
\forall y, z \in K, \quad|\alpha(y)-\alpha(z)| \leq L_{K}|y-z| .
$$

Then, for any $x_{0} \mathscr{F}_{0}$-measurable, there exists a unique maximal solution $X \mid[0, \zeta[$ of the SDE

$$
d X=\alpha(X) d Z, \quad X_{0}=x_{0} .
$$

Uniqueness holds in the sense that if $Y \mid\left[0, \xi\left[\right.\right.$ is another solution and $y_{0}=x_{0}$, then $\xi \leq \zeta$ a.s. and $X \mid[0, \xi[=Y$.

Proof. We proof is reduced to Proposition 2.4 by a standard truncation method. We briefly sketch the argument, since it will be used several times in the sequel. Let $B(0, R)=\left\{x \in \mathbb{R}^{n}:|x| \leq R\right\}$ where $R=1,2, \ldots$ and choose test functions $\phi_{R} \in C_{c}^{\infty}\left(\mathbb{R}^{n}\right)$ such that $\phi_{R} \mid B(0, R) \equiv 1$. For $R>0$ consider the "truncated SDE"

$$
d X^{R}=\alpha^{R}\left(X^{R}\right) d Z, \quad X_{0}^{R}=x_{0},
$$

where $\alpha^{R}:=\phi_{R} \alpha$ is now global Lipschitz. By Proposition 2.4 there is a unique solution $X^{R}$ to (2.4). Then

$$
X \mid\left[0, \tau_{R}\left[:=X^{R} \mid\left[0, \tau_{R}[\right.\right.\right.
$$

is well-defined by uniqueness, where

$$
\tau_{R}=\inf \left\{t \geq 0: X_{t}^{R} \notin B(0, R)\right\} .
$$

This finally defines $X$ on the stochastic interval $\left[0, \zeta\left[\right.\right.$ where $\zeta=\sup _{R} \tau_{R}$. Uniqueness of $X$ is deduced from the uniqueness of $X \mid\left[0, \tau_{R}[\right.$. 
Example 2.6. Consider the following Itô SDE on $\mathbb{R}^{n}$ :

$$
d X=\underbrace{\beta(X)}_{n \times 1} d t+\underbrace{\sigma(X)}_{n \times r} \underbrace{d B}_{r \times 1}
$$

where $B$ is Brownian motion on $\mathbb{R}^{r}$. Then the space-time process $Z_{t}=\left(t, B_{t}\right)$ is a semimartingale on $\mathbb{R}^{r+1}$ and SDE (2.5) can be written as

$$
d X=\left(\begin{array}{c}
\beta(X) \\
\sigma(X)
\end{array}\right)\left(\begin{array}{c}
d t \\
d B
\end{array}\right)=\alpha(X) d Z
$$

where $\alpha(X):=\left(\begin{array}{l}\beta(X) \\ \sigma(X)\end{array}\right)$. Thus, under a local Lipschitz condition on the coefficients $\beta$ and $\sigma$, the SDE

$$
d X=\beta(X) d t+\sigma(X) d B
$$

has a unique strong solution for every given initial condition $x_{0}$. By Proposition 2.3, maximal solutions of Eq. (2.6) are $L$-diffusions to the operator

$$
L=\sum_{i=1}^{n} \beta_{i} \partial_{i}+\frac{1}{2} \sum_{i, j=1}^{n}\left(\sigma \sigma^{*}\right)_{i j} \partial_{i} \partial_{j}
$$

where $\partial_{i}=\partial / \partial x_{i}$ is the derivative in direction $i$.

\subsection{Stratonovich differentials.}

Definition 2.7. For continuous real-valued semimartingales $X$ and $Y$ let

$$
X \circ d Y:=X d Y+\frac{1}{2} d[X, Y]
$$

be the Stratonovich differential. Here $X d Y$ is the usual Itô differential and $d[X, Y]=$ $d X d Y$ the differential of the quadratic covariation of $X$ and $Y$. The integral

$$
\int_{0}^{t} X \circ d Y=\int_{0}^{t} X d Y+\frac{1}{2}[X, Y]_{t}
$$

is called Stratonovich integral of $X$ with respect to $Y$.

Formula (2.7) gives the relation between the Stratonovich integral and the usual Itô integral. Since Stratonovich integrals can always be converted back to Itô integrals, their use in our context will be only formal and for the sake of convenient notations.

Remark 2.8. We have the following properties of Stratonovich differential, resp. Stratonovich integrals.

1. (Associativity) $X \circ(Y \circ d Z)=(X Y) \circ d Z$, i.e.,

$$
X \circ d\left(\int_{0}^{\cdot} Y \circ d Z\right)=(X Y) \circ d Z .
$$


Indeed, we have

$$
\begin{aligned}
X \circ(Y \circ d Z) & =X \circ d\left(\int_{0}^{\bullet} Y \circ d Z\right) \\
& =X d\left(\int_{0}^{\bullet} Y \circ d Z\right)+\frac{1}{2} d X d\left(\int_{0}^{\bullet} Y \circ d Z\right) \\
& =X(Y d Z)+\frac{1}{2} X d Y d Z+\frac{1}{2} d X\left(Y d Z+\frac{1}{2} d Y d Z\right) \\
& =(X Y) d Z+\frac{1}{2}(X d Y+Y d X+d X d Y) d Z \\
& =(X Y) d Z+\frac{1}{2} d(X Y) d Z \\
& =(X Y) \circ d Z .
\end{aligned}
$$

2. (Product rule) $d(X Y)=X \circ d Y+Y \circ d X$

Proof. By Itô's formula we have

$$
d(X Y)=X d Y+Y d X+d X d Y=X \circ d Y+Y \circ d X .
$$

Proposition 2.9 (Itô-Stratonovich formula). Let $X$ be a continuous $\mathbb{R}^{n}$-valued semimartingale and $f \in C^{3}\left(\mathbb{R}^{n}\right)$. Then

$$
d(f \circ X)=\sum_{i=1}^{n}\left(D_{i} f\right)(X) \circ d X^{i} \equiv\langle\nabla f(X), \circ d X\rangle .
$$

Proof. By Itô's formula, we have

$$
d\left(D_{i} f(X)\right)=\sum_{k=1}^{n}\left(D_{i} D_{k} f\right)(X) d X^{k}+\frac{1}{2} \sum_{k, \ell=1}^{n}\left(D_{i} D_{k} D_{\ell} f\right)(X) d X^{k} d X^{\ell} .
$$

Hence we get

$$
\begin{aligned}
\sum_{i=1}^{n}\left(D_{i} f\right)(X) \circ d X^{i} & =\sum_{i=1}^{n}\left(D_{i} f\right)(X) d X^{i}+\frac{1}{2} \sum_{i=1}^{n} d\left(D_{i} f(X)\right) d X^{i} \\
& =\sum_{i=1}^{n}\left(D_{i} f\right)(X) d X^{i}+\frac{1}{2} \sum_{i, k=1}^{n}\left(D_{i} D_{k} f(X)\right) d X^{k} d X^{i} \\
& =d(f \circ X) .
\end{aligned}
$$

Formula (2.8) shows the main advantage of the Stratonovich differential: it converts Itô's formula into the usual chain rule of classical analysis. Hence, at least formally, classical differential calculus can be applied in calculations involving Stratonovich differentials.

Proposition 2.10. Let $\beta: \mathbb{R}_{+} \times \mathbb{R}^{n} \rightarrow \mathbb{R}^{n}$ be continuous, $\sigma: \mathbb{R}_{+} \times \mathbb{R}^{n} \rightarrow \operatorname{Hom}\left(\mathbb{R}^{r}, \mathbb{R}^{n}\right)$ be $C^{1}$. Furthermore, let $B$ be a Brownian motion on $\mathbb{R}^{r}$. For a semimartingale $Y$ on $\mathbb{R}^{n}$ (defined up to some predictable stopping time $\tau$ ) the following conditions are equivalent:

(i) The semimartingale $Y$ is a solution of the Stratonovich SDE

$$
d Y=\beta(t, Y) d t+\sigma(t, Y) \circ d B,
$$

i.e.

$$
Y_{t}=Y_{0}+\int_{0}^{t} \beta\left(s, Y_{s}\right) d s+\int_{0}^{t} \sigma\left(s, Y_{s}\right) \circ d B_{s}, \quad \text { for } 0 \leq t<\tau \text { a.s. }
$$


(ii) For all $f \in C^{\infty}\left(\mathbb{R}^{n}\right)$,

$$
d(f \circ Y)=(L f)(t, Y) d t+\sum_{k=1}^{r}\left(A_{k} f\right)(t, Y) d B^{k} \quad \text { on }[0, \tau[
$$

where

$$
L=A_{0}+\frac{1}{2} \sum_{i=1}^{r} A_{k}^{2}
$$

with the vector fields $A_{i} \in \Gamma\left(T \mathbb{R}^{n}\right)$ defined as

$$
A_{0}=\sum_{i=1}^{n} \beta_{i} D_{i}, \quad A_{k}=\sum_{i=1}^{n} \sigma_{i k} D_{i}, \quad k=1, \ldots, r .
$$

Proof. (i) $\Rightarrow$ (ii) By the Itô-Stratonovich formula (Proposition 2.9) we have

$$
\begin{aligned}
d(f \circ Y) & =\sum_{i=1}^{n}\left(D_{i} f\right)(Y) \circ d Y^{i} \\
& =\sum_{i=1}^{n}\left(D_{i} f\right)(Y) \beta_{i}(t, Y) d t+\sum_{i=1}^{n}\left(D_{i} f\right)(Y)\left(\sum_{k=1}^{r} \sigma_{i k}(t, Y) \circ d B^{k}\right) \\
& =\left(A_{0} f\right)(t, Y) d t+\sum_{k=1}^{r}\left(A_{k} f\right)(t, Y) \circ d B^{k} \\
& =\left(A_{0} f\right)(t, Y) d t+\sum_{k=1}^{r}\left(A_{k} f\right)(t, Y) d B_{k}+\frac{1}{2} \sum_{k=1}^{r} d\left(\left(A_{k} f\right)(t, Y)\right) d B^{k} .
\end{aligned}
$$

Since

$$
d\left(A_{k} f(t, Y)\right)=\partial_{t}\left(A_{k} f\right)(t, Y) d t+\left(A_{0} A_{k} f\right)(t, Y) d t+\sum_{\ell=1}^{r}\left(A_{\ell} A_{k} f\right)(t, Y) \circ d B^{\ell},
$$

we observe that

$$
d\left(A_{k} f(t, Y)\right) d B^{k}=\left(A_{k}^{2} f\right)(t, Y) d t .
$$

and hence

$$
d(f \circ Y)=\underbrace{\left(\left(A_{0} f\right)(t, Y)+\frac{1}{2} \sum_{k=1}^{r}\left(A_{k}^{2} f\right)(t, Y)\right)}_{=(L f)(t, Y)} d t+\sum_{k=1}^{r}\left(A_{k} f\right)(t, Y) d B^{k} .
$$

(ii) $\Rightarrow$ (i) It is sufficient to take $f(x)=x_{\ell}$.

Corollary 2.11. Solutions to the Stratonovich SDE

$$
d Y=\beta(t, Y) d t+\sigma(t, Y) \circ d B
$$

define L-diffusions for the operator

$$
L=A_{0}+\frac{1}{2} \sum_{i=1}^{r} A_{i}^{2} \quad \text { with } A_{0}, A_{1}, \ldots, A_{r} \text { as in Eq. (2.10), }
$$

in the sense that

$$
d(f \circ Y)-(L f)(t, Y) d t \stackrel{\mathrm{m}}{=} 0
$$

for all $f \in C^{\infty}\left(\mathbb{R}^{n}\right)$. 
2.3. Stochastic differential equations on manifolds. In this section we describe the construction of $L$-diffusions as solutions of stochastic differential equations on manifolds $[18,27]$.

Definition 2.12. Let $M$ be a differentiable manifold, $\pi: T M \rightarrow M$ its tangent bundle and $E$ a finite dimensional vector space (without restrictions $E=\mathbb{R}^{r}$ ). A stochastic differential equation on $M$ is a pair $(A, Z)$ where

(1) $Z$ is a semimartingale taking values in $E$;

(2) $A: M \times E \rightarrow T M$ is a smooth homomorphism of vector bundles over $M$, i.e.

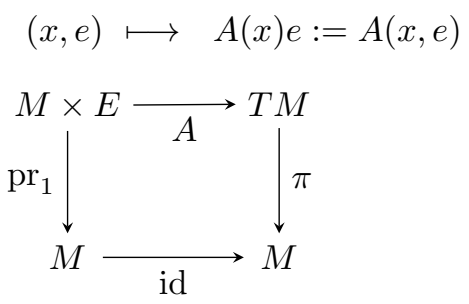

Remark 2.13. Formally the homomorphism $A$ may be considered as section $A \in$ $\Gamma\left(E^{*} \otimes T M\right)$. In particular, we have

$$
\begin{cases}\forall x \in M \text { fixed, } & A(x) \in \operatorname{Hom}\left(E, T_{x} M\right), \\ \forall e \in E \text { fixed, } & A(\cdot) e \in \Gamma(T M) .\end{cases}
$$

Notation 2.14. For the $\operatorname{SDE}(A, Z)$ we also write

$$
d X=A(X) \circ d Z
$$

or

$$
d X=\sum_{i=1}^{r} A_{i}(X) \circ d Z^{i}
$$

where $A_{i}=A(\cdot) e_{i} \in \Gamma(T M)$ and $e_{1}, \ldots, e_{r}$ is a basis of $E$.

Definition 2.15. Let $(A, Z)$ be an SDE on $M$ and let $x_{0}: \Omega \rightarrow M$ be $\mathscr{F}_{0^{-}}$ measurable. An adapted continuous process $X \mid\left[0, \zeta\left[\equiv\left(X_{t}\right)_{t<\zeta}\right.\right.$ taking values in $M$, defined up to the stopping time $\zeta$, is called solution to the SDE

$$
d X=A(X) \circ d Z
$$

with initial condition $X_{0}=x_{0}$, if for all $f \in C_{c}^{\infty}(M)$ the following conditions are satisfied:

(i) $f \circ X$ is a semimartingale;

(ii) for any stopping time $\tau$ such that $0 \leq \tau<\zeta$, we have

$$
f\left(X_{\tau}\right)=f\left(X_{0}\right)+\int_{0}^{\tau}(d f)_{X_{s}} A\left(X_{s}\right) \circ d Z_{s} .
$$

We call $X$ maximal solution of the SDE (2.11) if

$$
\{\zeta<\infty\} \subset\left\{\lim _{t \uparrow \zeta} X_{t}=\infty \text { in } \hat{M}=M \dot{\cup}\{\infty\}\right\} \text { a.s. }
$$

Note: The integral in (2.12) is defined using

$$
E \stackrel{A(x)}{\longrightarrow} T_{x} M \stackrel{(d f)_{x}}{\longrightarrow} \mathbb{R}, \quad x \in M .
$$


Remark 2.16. We adopt the convention $X_{t}(\omega):=\infty$ for $\zeta(\omega) \leq t<\infty$ and $f(\infty)=0$ for $f \in C_{c}^{\infty}(M)$. Then we may write, for all $t \geq 0$,

$$
\begin{aligned}
f\left(X_{t}\right) & =f\left(X_{0}\right)+\int_{0}^{t}(d f)_{X_{s}} A\left(X_{s}\right) \circ d Z_{s} \\
& =f\left(X_{0}\right)+\sum_{i=1}^{r} \int_{0}^{t}(d f)_{X_{s}} A_{i}\left(X_{s}\right) \circ d Z_{s}^{i} \\
& =f\left(X_{0}\right)+\sum_{i=1}^{r} \int_{0}^{t}\left(A_{i} f\right)\left(X_{s}\right) \circ d Z_{s}^{i} \quad \text { with } A_{i}=A(\cdot) e_{i} .
\end{aligned}
$$

Example 2.17. Let $E=\mathbb{R}^{r+1}$ and $Z=\left(t, Z^{1}, \ldots, Z^{r}\right)$ where $\left(Z^{1}, \ldots, Z^{r}\right)$ is a Brownian motion on $\mathbb{R}^{r}$. Denote the standard basis of $\mathbb{R}^{r+1}$ by $\left(e_{0}, e_{1}, \ldots, e_{r}\right)$. Letting

$$
A: M \times E \rightarrow T M
$$

be a homomorphism of vector bundles over $M$, we consider the vector fields

$$
A_{i}:=A(\cdot) e_{i} \in \Gamma(T M), \quad i=0,1, \ldots, r .
$$

Then the SDE

$$
d X=A(X) \circ d Z
$$

may be written as

$$
d X=A_{0}(X) d t+\sum_{i=1}^{r} A_{i}(X) \circ d Z^{i}
$$

and for each $f \in C_{c}^{\infty}(M)$ we have

$$
\begin{aligned}
d(f \circ X) & =(d f)_{X} A(X) \circ d Z \\
& =\sum_{i=0}^{r}(d f)_{X} A(X) e_{i} \circ d Z^{i} \\
& =\sum_{i=0}^{r}(d f)_{X} A_{i}(X) \circ d Z^{i} \\
& =\sum_{i=0}^{r}\left(A_{i} f\right)(X) \circ d Z^{i} \\
& =\left(A_{0} f\right)(X) d t+\sum_{i=1}^{r}\left(A_{i} f\right)(X) \circ d Z^{i} \\
& =\left(A_{0} f\right)(X) d t+\sum_{i=1}^{r}\left[\left(A_{i} f\right)(X) d Z^{i}+\frac{1}{2} d\left(\left(A_{i} f\right)(X)\right) d Z^{i}\right] .
\end{aligned}
$$

Taking into account that

$$
d\left(\left(A_{i} f\right)(X)\right)=\sum_{j=1}^{r}\left(A_{j} A_{i} f\right)(X) d Z^{j}+d(\text { terms of bounded variation })
$$

we see that

$$
d\left(\left(A_{i} f\right)(X)\right) d Z^{i}=\left(A_{i}^{2} f\right)(X) d t
$$


where we used that $d Z^{i} d Z^{j}=\delta_{i j} d t$ for $1 \leq i, j \leq r$. Hence we get

$$
\begin{aligned}
d(f \circ X) & =\left(A_{0} f\right)(X) d t+\frac{1}{2} \sum_{j=1}^{r}\left(A_{i}^{2} f\right)(X) d t+\sum_{i=1}^{r}\left(A_{i} f\right)(X) d Z^{i} \\
& =(L f)(X) d t+\sum_{i=1}^{r}\left(A_{i} f\right)(X) d Z^{i} .
\end{aligned}
$$

Corollary 2.18. Let $L=A_{0}+\frac{1}{2} \sum_{i=1}^{r} A_{i}^{2}$ and let $X$ be a solution to Eq. (2.13). Then, for all $f \in C_{c}^{\infty}(M)$,

$$
d(f \circ X)-(L f)(X) d t \stackrel{\mathrm{m}}{=} 0
$$

where $\stackrel{\mathrm{m}}{=}$ denotes equality modulo differentials of martingales. In other words, maximal solutions to the $\mathrm{SDE}$

$$
d X=A(X) \circ d Z
$$

are L-diffusions to the operator $L=A_{0}+\frac{1}{2} \sum_{i=1}^{r} A_{i}^{2}$.

Theorem 2.19 (SDE: Existence and uniqueness of solutions; $\left.M=\mathbb{R}^{n}\right)$. Let $(A, Z)$ be an $\mathrm{SDE}$ on $M=\mathbb{R}^{n}$ and $x_{0}$ an $\mathscr{F}_{0}$-measurable random variable taking values in $\mathbb{R}^{n}$. Then there exists a unique maximal solution $X$ (with maximal lifetime $\zeta>0$ a.s.) of the $\mathrm{SDE}$

$$
d X=A(X) \circ d Z
$$

with initial condition $X_{0}=x_{0}$. Uniqueness holds in the following sense: if $Y \mid[0, \xi[$ is another solution of (2.14) to the same initial condition, then $\xi \leq \zeta$ a.s. and $X \mid[0, \xi[=Y$ a.s.

Proof. As in the proof of Proposition 2.5 let $B(0, R)=\left\{x \in \mathbb{R}^{n}:|x| \leq R\right\}$ where $R=1,2, \ldots$ and choose test functions $\phi_{R} \in C_{c}^{\infty}\left(\mathbb{R}^{n}\right)$ such that $\phi_{R} \mid B(0, R) \equiv 1$. Since

$$
A \in \Gamma\left(\operatorname{Hom}\left(\mathbb{R}^{r}, T M\right)\right),
$$

we have for each $x \in \mathbb{R}^{n}$ the linear map

$$
A(x): \mathbb{R}^{r} \rightarrow T_{x} M .
$$

In this way $A$ gives rise to a smooth map $\mathbb{R}^{n} \rightarrow \operatorname{Matr}(n \times r ; \mathbb{R})$.

Consider now the "truncated SDE"

$$
d X^{R}=A^{R}\left(X^{R}\right) \circ d Z
$$

where $A^{R}=\phi_{R} A$. By Proposition 2.4, the truncated SDE (2.15) has a unique global solution $X^{R}$ with initial condition $X_{0}^{R}=x_{0}$, i.e., for each $R$ there exists a continuous $\mathbb{R}^{n}$-valued semimartingale $\left(X_{t}^{R}\right)_{t \geq 0}$ satisfying $X_{0}^{R}=x_{0}$ such that (2.15) holds in the Itô-Stratonovich sense. In terms of the stopping times

$$
\tau_{R}:=\inf \left\{t \geq 0: X_{t}^{R} \notin B(0, R)\right\},
$$

we have for $R<R^{\prime}$,

$$
X^{R^{\prime}} \mid\left[0, \tau_{R}\left[=X^{R} \mid\left[0, \tau_{R}[\quad \text { a.s. }\right.\right.\right.
$$

Hence a stochastic process $X$ (with lifetime $\zeta=\lim _{R \uparrow \infty} \tau_{R}$ ) is well-defined via

$$
X \mid\left[0, \tau_{R}\left[=X^{R} \mid\left[0, \tau_{R}[.\right.\right.\right.
$$


For each $f \in C_{c}^{\infty}\left(\mathbb{R}^{n}\right)$ such that $\operatorname{supp}(f) \subset B(0, R)$ (with $R$ sufficiently large), we have

$$
\begin{aligned}
d(f \circ X) & =d\left(f \circ X^{R}\right) \\
& =\sum_{k=1}^{n}\left(D_{k} f\left(X^{R}\right)\right) \circ d\left(X^{R}\right)^{k} \quad \text { using Itô-Stratonovich formula) } \\
& =\left\langle\nabla f\left(X^{R}\right), \circ d X^{R}\right\rangle \\
& =\left\langle\nabla f\left(X^{R}\right), \phi_{R}\left(X^{R}\right) A\left(X^{R}\right) \circ d Z\right\rangle \\
& =\langle\nabla f(X), A(X) \circ d Z\rangle \\
& =\sum_{i=1}^{r}\left\langle\nabla f(X), A_{i}(X) \circ d Z^{i}\right\rangle \\
& =\sum_{i=1}^{r}(d f)_{X} A_{i}(X) \circ d Z^{i} \\
& =(d f)_{X} A(X) \circ d Z .
\end{aligned}
$$

Hence, $X$ is the unique solution to Eq. (2.14) with initial condition $X_{0}=x_{0}$. Note that $X$ is a solution of $d X=A(X) \circ d Z$ in the Itô-Stratonovich sense (in $\mathbb{R}^{n}$ ) if and only if $\forall f \in C_{c}^{\infty}\left(\mathbb{R}^{n}\right)$,

$$
d(f \circ X)=(d f)_{X} A(X) \circ d Z .
$$

Theorem 2.20 (SDE: Existence and uniqueness of solutions; general case). Let $(A, Z)$ be an $\mathrm{SDE}$ on a differentiable manifold $M$ and let $x_{0}: \Omega \rightarrow M$ be $\mathscr{F}_{0}$ measurable. There exists a unique maximal solution $X \mid[0, \zeta[$ (where $\zeta>0$ a.s.) of the SDE

$$
d X=A(X) \circ d Z
$$

with initial condition $X_{0}=x_{0}$. Uniqueness holds in the sense that if $Y \mid[0, \xi[$ is another solution with $Y_{0}=x_{0}$, then $\xi \leq \zeta$ a.s. and $X \mid[0, \xi[=Y$ a.s.

We shall reduce Theorem 2.20 to Theorem 2.19 via embedding the manifold $M$ into a high-dimensional Euclidean space.

WhitNEY'S EMBEDDING THEOREM. Each manifold $M$ of dimension $n$ can be embedded into $\mathbb{R}^{n+k}$ as a closed submanifold (for $k$ sufficiently large, e.g. $k=n+1$ ), i.e.,

$$
M \hookrightarrow \iota(M) \subset \mathbb{R}^{n+k}
$$

where $\iota: M \rightarrow \iota(M)$ is a diffeomorphism and $\iota(M) \subset \mathbb{R}^{n+k}$ a closed submanifold.

Proof (of Theorem 2.20). We choose a Whitney embedding (in general not intrinsic)

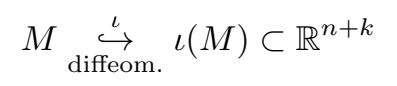

and identify $M$ and $\iota(M)$; in particular for each $x \in M$ the tangent space $T_{x} M$ is then a linear subspace of $\mathbb{R}^{n+k}$ according to

$$
T_{x} M \stackrel{d \iota_{x}}{\hookrightarrow} T_{x} \mathbb{R}^{n+k} \equiv \mathbb{R}^{n+k} .
$$

Vector fields $A_{1}, \ldots, A_{r} \in \Gamma(T M)$ can be extended to vector fields

$$
\bar{A}_{1}, \ldots, \bar{A}_{r} \in \Gamma\left(T \mathbb{R}^{n+k}\right) \equiv C^{\infty}\left(\mathbb{R}^{n+k} ; \mathbb{R}^{n+k}\right) \quad \text { with } \bar{A}_{i} \mid M=A_{i},
$$

i.e. $\bar{A}_{i} \circ \iota=d \iota \circ A_{i}$. Hence a given bundle map

$$
A: M \times \mathbb{R}^{r} \rightarrow T M, \quad(x, z) \mapsto A(x) z=\sum_{i=1}^{r} A_{i}(x) z^{i}
$$


has a continuation

$$
\bar{A}: \mathbb{R}^{n+k} \times \mathbb{R}^{r} \rightarrow \mathbb{R}^{n+k} \times \mathbb{R}^{n+k}, \quad(x, z) \mapsto \bar{A}(x) z=\sum_{i=1}^{r} \bar{A}_{i}(x) z^{i} .
$$

The idea is to consider in place of the original SDE

$$
d X=A(X) \circ d Z \text { on } M
$$

the SDE

$$
d X=\bar{A}(X) \circ d Z \text { on } \mathbb{R}^{n+k} .
$$

First of all it is clear that any solution of $(*)$ in $M$ provides a solution of $(\bar{*})$ in $\mathbb{R}^{n+k}$. More precisely: If $X$ is a solution to $(*)$ with starting value $X_{0}=x_{0}$, then $\bar{X}:=\iota \circ X$ solves equation $(\bar{*})$ with starting value $\bar{X}_{0}=\iota \circ x_{0}$. Indeed if $\bar{f} \in C_{c}^{\infty}\left(\mathbb{R}^{n+k}\right)$, then $f:=\bar{f} \mid M=\bar{f} \circ \iota \in C_{c}^{\infty}(M)$, and we have:

$$
\begin{aligned}
d(\bar{f} \circ \bar{X})=d(f \circ X) & =\sum_{i=1}^{r}(d f)_{X} A_{i}(X) \circ d Z^{i} \\
& =\sum_{i=1}^{r}(d \bar{f})_{\bar{X}}(d \iota)_{X} A_{i}(X) \circ d Z^{i} \\
& =\sum_{i=1}^{r}(d \bar{f})_{\bar{X}} \bar{A}_{i}(\iota \circ X) \circ d Z^{i} \\
& =\sum_{i=1}^{r}(d \bar{f})_{\bar{X}} \bar{A}_{i}(\bar{X}) \circ d Z^{i} .
\end{aligned}
$$

This implies in particular uniqueness of solutions to $(*)$, since equation $(\bar{*})$ has a unique solution to a given initial condition.

To establish existence of solutions to $(*)$ we first remark that any test function $f \in C_{c}^{\infty}(M)$ has a continuation $\bar{f} \in C_{c}^{\infty}\left(\mathbb{R}^{n+k}\right)$ such that $\bar{f} \mid M \equiv \bar{f} \circ \iota=f$. We make the following important observation.

Each solution $X \mid\left[0, \zeta\left[\right.\right.$ of $(\bar{*})$ in $\mathbb{R}^{n+k}$ with $X_{0}=x_{0}$ which stays on $M$ for $t<\zeta$ (where $x_{0}$ is an $M$-valued $\mathscr{F}_{0}$-measurable random variable) gives a solution of $(*)$.

Hence, to complete the proof it is sufficient to show the following lemma.

Lemma 2.21. If $X \mid\left[0, \zeta\left[\right.\right.$ is the maximal solution of $(\bar{*})$ in $\mathbb{R}^{n+k}$ with $X_{0}=x_{0}$, then

$$
\{t<\zeta\} \subset\left\{X_{t} \in M\right\}, \text { for all } t \text { a.s. }
$$

Observe that it is enough to verify Lemma 2.21 for one specific continuation $\bar{A}$ of $A$.

Proof (of Lemma 2.21). Let

$$
\perp M=\left\{(x, v) \in M \times \mathbb{R}^{n+k} \mid v \in\left(T_{x} M\right)^{\perp}\right\},
$$

be the normal bundle of $M$ and consider $M$ embedded into $\perp M$ as zero section:

$$
M \hookrightarrow \perp M, \quad x \mapsto(x, 0)
$$




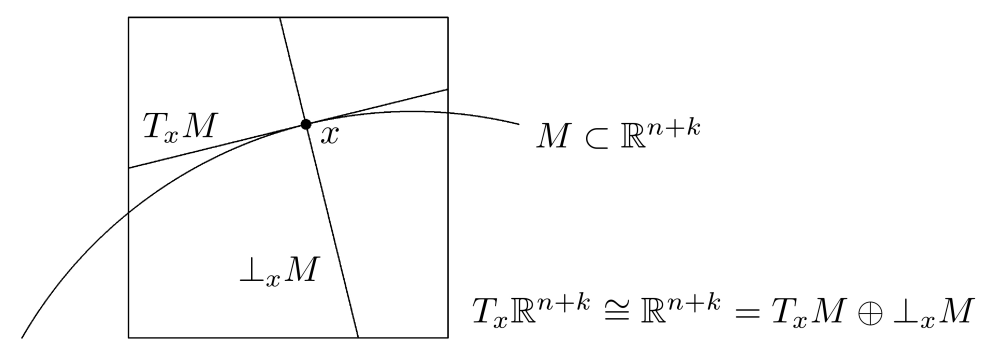

Fact: There is a smooth function $\varepsilon: M \rightarrow] 0, \infty[$ such that the map

$$
\begin{aligned}
\tau_{\varepsilon}(M):=\{(x, v) \in \perp M:|v|<\varepsilon(x)\} & \stackrel{\cong}{\longrightarrow} \bigcup_{x \in M}\left\{y \in \mathbb{R}^{n+k}:|y-x|<\varepsilon(x)\right\}, \\
(x, v) & \longmapsto x+v,
\end{aligned}
$$

is a diffeomorphism from the tubular neighbourhood $\tau_{\varepsilon}(M)$ of $M$ of radius $\varepsilon$ onto the indicated part in $\mathbb{R}^{n+k}$. This follows from the local inversion theorem since the given map has full rank along the zero section of $\perp M$.

Note that both

$$
\begin{aligned}
\pi: \tau_{\varepsilon}(M) & \rightarrow M, \quad(x, v) \mapsto x \\
\operatorname{dist}^{2}(\cdot, M): \tau_{\varepsilon}(M) & \rightarrow \mathbb{R}, \quad(x, v) \mapsto|v|^{2},
\end{aligned}
$$

are smooth maps.

Now letting $R>0$ be sufficiently large such that

$$
M \cap B(0, R+1) \neq \varnothing
$$

then

$$
\varepsilon_{R}=\inf \{\varepsilon(x) \mid x \in M \cap B(0, R+1)\}>0 .
$$

We choose a decreasing smooth function $\lambda:[0, \infty[\rightarrow[0,1]$ of the form

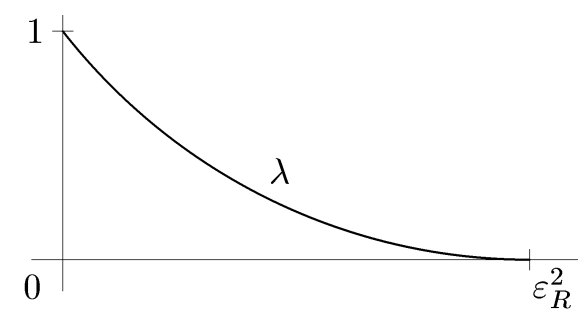

and a test function $0 \leq \varphi \in C_{c}^{\infty}\left(\mathbb{R}^{n+k}\right)$ such that $\varphi \mid B(0, R) \equiv 1$ and $\operatorname{supp}(\varphi) \subset$ $B(0, R+1)$. Consider the map

$$
\begin{aligned}
& \bar{A}^{R}: \mathbb{R}^{n+k} \times \mathbb{R}^{r} \rightarrow \mathbb{R}^{n+k} \times \mathbb{R}^{n+k}, \\
& \bar{A}^{R}(y, z):= \begin{cases}\varphi(y) \lambda\left(\operatorname{dist}^{2}(y, M)\right) A(\pi(y)) z & \text { if } y \in \tau_{\varepsilon}(M), \\
0 & \text { if } y \notin \tau_{\varepsilon}(M) .\end{cases}
\end{aligned}
$$




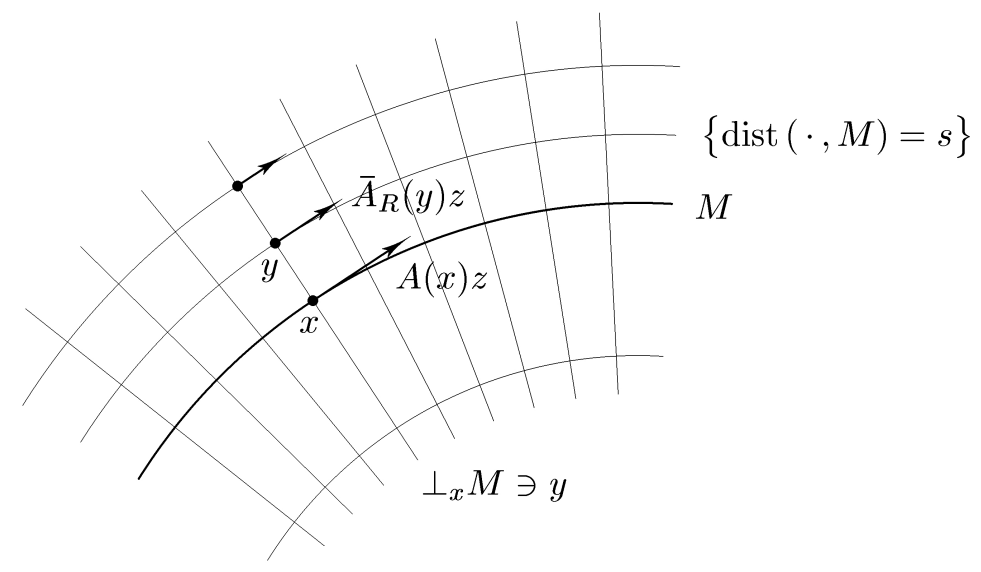

Let $X$ be the solution of

$$
d X=\bar{A}^{R}(X) \circ d Z, \quad X_{0}=x_{0} .
$$

Consider the test function $f \in C_{c}^{\infty}\left(\mathbb{R}^{n+k}\right)$ given as

$$
f(y)=\varphi(y) \lambda\left(\operatorname{dist}^{2}(y, M)\right)
$$

Then

$$
\begin{aligned}
d(f \circ X) & =(d f)_{X} \bar{A}^{R}(X) \circ d Z \\
& =\left\langle\nabla f(X), \bar{A}^{R}(X) \circ d Z\right\rangle \\
& =0 \quad \text { on }\left[0, \tau_{R}[,\right.
\end{aligned}
$$

where $\tau_{R}:=\inf \left\{t \geq 0: X_{t} \notin B(0, R)\right\}$. Indeed, $f$ is constant on each submanifold of the form

$$
\{\operatorname{dist}(\cdot, M)=s\} \cap B(0, R), \quad s<\varepsilon_{R},
$$

whereas $\bar{A}^{R}(y, z)$ is tangent to such submanifolds. Thus, for all $y \in B(0, R)$ and $z \in \mathbb{R}^{r}$,

$$
\nabla f(y) \perp \bar{A}^{R}(y) z .
$$

Hence, for any solution $X$ of $(\bar{*})$, we obtain that

$$
f(X) \equiv \text { constant on }\left[0, \tau_{R}[\text { a.s. }\right.
$$

Since $R$ is arbitrary, this completes the proof of the Lemma.

Solutions to an SDE on $M$ of the type (2.11) are by definition semimartingales on $M$ as defined above: A continuous adapted process $X$ with values in $M$ is a semimartingale on $M$ if, for each $f \in C_{c}^{\infty}(M)$, the composition $f \circ X$ provides a continuous real-valued semimartingale. It is easy to see that each $M$-valued semimartingale can be obtained as solution of an SDE on $M$.

Theorem 2.22 (Manifold-valued semimartingales as solutions of an SDE). Every semimartingale on a manifold $M$ is given as solution of an $\mathrm{SDE}$ of the type (2.11).

Proof. Let $X$ be an arbitrary semimartingale on $M$. Without loss of generality (after an eventual change of time), we may assume that $X$ has infinite lifetime. Choosing a Whitney embedding $\iota: M \hookrightarrow \mathbb{R}^{n+k}$ we may consider the semimartingale $Z:=\iota \circ X$ taking values in $E:=\mathbb{R}^{n+k}$. Let $A: M \times E \rightarrow T M$ be the bundle homomorphism which is fibrewise the orthogonal projection $A(x): \mathbb{R}^{n+k} \rightarrow T_{x} M$ of $\mathbb{R}^{n+k}$ onto $T_{x} M \subset T_{x} \mathbb{R}^{n+k}=\mathbb{R}^{n+k}$. We show that $X$ solves the equation

$$
d X=A(X) \circ d Z \text {. }
$$


Let $f \in C_{c}^{\infty}(M)$ be given. We choose a continuation $\bar{f} \in C_{c}^{\infty}\left(\mathbb{R}^{n+k}\right)$ where $\bar{f} \circ \iota=f$ such that $\bar{f}$ is constant locally about $M$ on the normal subspaces $\perp_{x} M$ (this is $\bar{f}(y)=f(x)$ for $y \in \perp_{x} M$ sufficiently small). Now let $x \in M$ and $z \in \mathbb{R}^{n+k}$. By decomposing $z=z_{0}+z^{\perp}$ where $z_{0} \in T_{x} M$ and $z^{\perp} \in \perp_{x} M$, we obtain:

$$
(d f)_{x} A(x) z=(d \bar{f})_{\iota(x)}(d \iota)_{x} A(x) z=(d \bar{f})_{\iota(x)} z_{0}=(d \bar{f})_{\iota(x)} z .
$$

But then

$$
\begin{aligned}
d(f \circ X) & =d(\bar{f} \circ \iota \circ X)=\sum_{i=1}^{n+k}\left(D_{i} \bar{f}\right)(\iota \circ X) \circ d Z^{i} \\
& =\sum_{i=1}^{n+k}(d f)_{X} A(X) e_{i} \circ d Z^{i}=(d f)_{X} A(X) \circ d Z
\end{aligned}
$$

which gives the claim.

\section{Some PRobabilistic Formulas For SOlutions of PDEs}

Let $L$ be a second order partial differentiable operator on $M$, e.g. $M$ a general differentiable manifold and $L$ given in so-called "Hörmander form" as

$$
L=A_{0}+\frac{1}{2} \sum_{i=1}^{r} A_{i}^{2}
$$

For $x \in M$, let $X_{t}(x)$ be an $L$-diffusion, starting from $x$ at time $t=0$, i.e. $X_{0}(x)=$ $x$. Recall that $X_{t}(x)$ can be constructed as the solution to the SDE on $M$,

$$
\left\{\begin{array}{l}
d X=A_{0}(X) d t+\sum_{i=1}^{r} A_{i}(X) \circ d B^{i}, \\
X_{0}=x
\end{array}\right.
$$

where $B$ denotes Brownian motion on $\mathbb{R}^{r}$. Sometimes one starts with a partial differentiable operators $L$ on $M$ which locally in a chart $(h, U)$ writes as

$$
L \mid U=\sum_{i=1}^{n} b_{i} \partial_{i}+\sum_{i, j=1}^{n}\left(\sigma \sigma^{*}\right)_{i j} \partial_{i} \partial_{j},
$$

where $b \in C^{\infty}\left(U, \mathbb{R}^{n}\right)$ and $a \in C^{\infty}\left(U, \mathbb{R}^{r} \otimes \mathbb{R}^{n}\right)$ (using the notation $\partial_{i}=\frac{\partial}{\partial h_{i}}$ ). It is straight-forward to rewrite such a an operator in "Hörmander form" (3.1) and then to construct an $L$-diffusion by solving a Stratonovich SDE.

In the special case $M=\mathbb{R}^{n}$ and

$$
L=\sum_{i=1}^{n} b_{i} \partial_{i}+\frac{1}{2} \sum_{i, j=1}^{n}\left(\sigma \sigma^{*}\right)_{i j} \partial_{i} \partial_{j},
$$

an $L$-diffusion can be constructed directly as solution of the Itô SDE on $\mathbb{R}^{n}$ :

$$
\left\{\begin{array}{l}
d X=b(X) d t+\sigma(X) d B \\
X_{0}=x
\end{array}\right.
$$

where $B$ is again a Brownian motion on $\mathbb{R}^{r}$. 
3.1. Feynman-Kac formula. Let $L$ be as in Eq. (3.1). Suppose that the lifetime of $X_{t}(x)$ is infinite a.s. for all $x \in M$.

Proposition 3.1 (Feynman-Kac formula). Let $f: M \rightarrow \mathbb{R}$ be continuous and bounded and $V: M \rightarrow \mathbb{R}$ be continuous and bounded above, i.e. $V(x) \leq K$ for some constant $K>0$. Let $u: \mathbb{R}_{+} \times M \rightarrow \mathbb{R}$ be a bounded solution of the following "initial value problem"

$$
\left\{\begin{array}{l}
\frac{\partial}{\partial t} u=L u+V u \\
\left.u\right|_{t=0}=f,
\end{array}\right.
$$

i.e.

$$
\left\{\begin{array}{l}
\frac{\partial}{\partial t} u(t, \cdot)=L u(t, \cdot)+V(\cdot) u(t, \cdot) \\
u(0, \cdot)=f(\cdot) .
\end{array}\right.
$$

Then the solution $u$ is given by the formula

$$
u(t, x)=\mathbb{E}\left[\exp \left(\int_{0}^{t} V\left(X_{s}(x)\right) d s\right) f\left(X_{t}(x)\right)\right] .
$$

Remark 3.2. Operators of the form $H=L+V$ (where $V$ is the multiplication operator by $V$ ) are called Schrödinger operators, for instance, $H=\frac{1}{2} \triangle+V$. The function $V$ is called potential. If $H$ is (essentially) self-adjoint, then

$$
u(t, \cdot)=e^{t H} f
$$

by semigroup theory.

Proof (of Proposition 3.1). Fix $t>0$ and consider the process $Y_{s}:=A_{s} Z_{s}$ where

$$
\left\{\begin{array}{l}
A_{s}:=\exp \left(\int_{0}^{s} V\left(X_{r}(x)\right) d r\right) \\
Z_{s}:=u\left(t-s, X_{s}(x)\right)
\end{array}\right.
$$

We will show that $\left(Y_{s}\right)_{0 \leq s \leq t}$ is a martingale in our setting.

Indeed: First note that by Itô's formula

$$
d Z_{s}=\left(\partial_{s} u(t-s, \cdot)+L u(t-s, \cdot)\right)\left(X_{s}(x)\right) d s+d N_{s}
$$

where $N_{s}$ is local martingale. Thus, since $A_{s}$ is of bounded variation, we have

$$
\begin{aligned}
d Y_{s} & =Z_{s} d A_{s}+A_{s} d Z_{s} \\
& =Z_{s} A_{s} V\left(X_{s}(x)\right) d s+A_{s}\left(\partial_{s} u(t-s, \cdot)+L u(t-s, \cdot)\right)\left(X_{s}(x)\right) d s+A_{s} d N_{s} \\
& =A_{s} \underbrace{\left(-\partial_{t} u+L u+V u\right)}_{=0}\left(t-s, X_{s}(x)\right) d s+A_{s} d N_{s} .
\end{aligned}
$$

Hence $\left(Y_{s}\right)_{0 \leq s \leq t}$ is a local martingale, and as it is bounded, $\left(Y_{s}\right)_{0 \leq s \leq t}$ is a true martingale. In particular, by taking expectations we obtain

$$
\begin{aligned}
u(t, x)=\mathbb{E}\left[Y_{0}\right]=\mathbb{E}\left[Y_{t}\right] & =\mathbb{E}\left[\exp \left(\int_{0}^{t} V\left(X_{r}(x)\right) d r\right) u\left(0, X_{t}(x)\right)\right] \\
& =\mathbb{E}\left[\exp \left(\int_{0}^{t} V\left(X_{r}(x)\right) d r\right) f\left(X_{t}(x)\right)\right] .
\end{aligned}
$$


3.2. Elliptic boundary value problems. Let $L$ be a second order partial differential operator on a differential manifold $M$, e.g.

$$
\begin{aligned}
& L=A_{0}+\frac{1}{2} \sum_{i=1}^{r} A_{i}^{2} \quad \text { on a differential manifold } M, \text { or } \\
& L=\sum_{i=1}^{n} b_{i} \partial_{i}+\frac{1}{2} \sum_{i, j=1}^{n}\left(\sigma \sigma^{*}\right)_{i j} \partial_{i} \partial_{j} \quad \text { in local coordinates on } M .
\end{aligned}
$$

Remark 3.3 (Ellipticity).

(1) The "diffusion vector fields" $A_{1}, \ldots, A_{r}$ define for each $x \in M$ a linear map

$$
A(x): \mathbb{R}^{r} \rightarrow T_{x} M, \quad z \mapsto \sum_{i=1}^{r} A_{i}(x) z_{i} .
$$

The operator

$$
L=A_{0}+\frac{1}{2} \sum_{i=1}^{r} A_{i}^{2}
$$

is called elliptic on some subset $D \subset M$ if the map $A(x)$ is surjective for each $x \in D$.

(2) Similarly, an operator of the type

$$
L=\sum_{i=1}^{n} b_{i} \partial_{i}+\frac{1}{2} \sum_{i, j=1}^{n}\left(\sigma \sigma^{*}\right)_{i j} \partial_{i} \partial_{j}
$$

is called elliptic on some subset $D \subset M$ if the linear map

$$
\sigma(x): \mathbb{R}^{r} \rightarrow \mathbb{R}^{n}, \quad z \mapsto \underbrace{\sigma(x)}_{n \times r} z,
$$

is surjective for each $x \in D$.

It is easily checked that both notions of ellipticity are compatible.

Note 3.4. The following conditions are equivalent:

$$
\begin{aligned}
\sigma(x) \text { is surjective } & \Longleftrightarrow \sigma^{*}(x) \text { is injective } \\
& \Longleftrightarrow a(x):=\sigma(x) \sigma^{*}(x) \text { is invertible } \\
& \Longleftrightarrow\langle a(x) v, v\rangle>0, \quad \forall 0 \neq v \in \mathbb{R}^{n} .
\end{aligned}
$$

Example 3.5 (Expected hitting time of a boundary). Let $\varnothing \neq D \subsetneq M$ be some open, relatively compact domain with boundary $\partial D$. Suppose that there exists a solution $u \in C^{2}(D) \cap C(\bar{D})$ to the problem

$$
\left\{\begin{array}{l}
L u=-1 \text { on } D, \\
\left.u\right|_{\partial D}=0 .
\end{array}\right.
$$

(For instance, if $L$ is elliptic on $\bar{D}$ and the boundary $\partial D$ is smooth, it is well-known by classical PDE theory that such a solution exists).

Let $X_{t}(x)$ be an $L$-diffusion such that $X_{0}(x)=x$ and denote by

$$
\tau_{D}(x)=\inf \left\{t>0: X_{t}(x) \in \partial D\right\}
$$

Then, for each $x \in D$,

$$
u(x)=\mathbb{E}\left[\tau_{D}(x)\right]
$$

In particular, we see that $u>0$ on $D$. 
Proof. For $x \in D$, let $X_{t}=X_{t}(x)$ and $\tau_{D}=\tau_{D}(x)$. We know that the process

$$
u\left(X_{t \wedge \tau_{D}}\right)-u(x)-\int_{0}^{t \wedge \tau_{D}} L u\left(X_{s}\right) d s, \quad t \geq 0,
$$

is a martingale (starting at 0 ), and hence

$$
\mathbb{E}\left[u\left(X_{t \wedge \tau_{D}}\right)\right]-u(x)=\mathbb{E}[\int_{0}^{t \wedge \tau_{D}} \underbrace{L u\left(X_{s}\right)}_{=-1} d s] .
$$

This shows that

$$
\mathbb{E}\left[t \wedge \tau_{D}\right]=u(x)-\mathbb{E}\left[u\left(X_{t \wedge \tau_{D}}\right)\right] .
$$

Recall that $u$ is bounded, since $u \in C(\bar{D})$ with $\bar{D}$ compact, and hence by BeppoLevi,

$$
\mathbb{E}\left[\tau_{D}\right]=\lim _{t \rightarrow \infty} \mathbb{E}\left[t \wedge \tau_{D}\right]<+\infty
$$

Thus, by letting $t \uparrow+\infty$ in (3.4), we obtain

$$
\mathbb{E}\left[\tau_{D}\right]=u(x)-\mathbb{E}\left[u\left(X_{\tau_{D}}\right)\right]=u(x),
$$

where we used that $u \mid \partial D=0$.

Corollary 3.6. If the boundary value problem smooth (3.3) has a solution, then $\mathbb{E}\left[\tau_{D}(x)\right]<\infty$, and hence $\tau_{D}(x)<\infty$ a.s. for all $x \in D$. Thus L-diffusions starting at any point $x \in D$ eventually hit $\partial D$ with probability 1 .

Remark 3.7. The property of an $L$-diffusion of hitting the boundary with probability 1 is a "non-degeneracy" condition on the operator $L$. We demonstrate this in the following simple example on $\mathbb{R}^{n}$.

Example 3.8. Consider an operator of the form

$$
L=\sum_{i=1}^{n} b_{i} \partial_{i}+\frac{1}{2} \sum_{i, j=1}^{n}\left(\sigma \sigma^{*}\right)_{i j} \partial_{i} \partial_{j} \quad \text { on } \mathbb{R}^{n}, \quad a_{i j}=\left(\sigma \sigma^{*}\right)_{i j},
$$

and let with $D \subset \mathbb{R}^{n}$ be relatively compact. Suppose that $L$ is non-degenerate in the following weak sense: for some $1 \leq \ell \leq n$ there holds

$$
\min _{x \in \bar{D}} a_{\ell \ell}(x)>0
$$

Then $\mathbb{E}\left[\tau_{D}(x)\right]<\infty$ for any $x \in D$.

Proof. Set

$$
A:=\min _{x \in \bar{D}} a_{\ell \ell}(x) \quad \text { and } \quad B:=\max _{x \in \bar{D}}|b(x)| .
$$

For constants $\mu, \nu>0$ consider the smooth function

$$
h(x)=-\mu e^{\nu x_{\ell}}, \quad x \in D .
$$

Then, choosing $\nu>2 B / A$ and taking $K=\min _{x \in \bar{D}} x_{\ell}$, we get

$$
\begin{aligned}
-L h(x) & =\mu e^{\nu x_{\ell}}\left(\frac{\nu^{2}}{2} a_{\ell \ell}(x)+\nu b_{\ell}(x)\right) \\
& \geq \frac{1}{2} \mu \nu A e^{\nu x_{\ell}}\left(\nu-\frac{2 B}{A}\right) \\
& \geq \frac{1}{2} \nu \mu A e^{\nu K}\left(\nu-\frac{2 B}{A}\right) \\
& \geq 1 \quad \text { for } \mu \text { sufficiently large. }
\end{aligned}
$$


Thus

$$
L h \leq-1 \text { on } D
$$

As above, we may proceed as follows. The process

$$
N_{t}^{h}:=h\left(X_{t \wedge \tau_{D}}\right)-h(x)-\int_{0}^{t \wedge \tau_{D}} L h\left(X_{s}\right) d s, \quad t \geq 0
$$

is a martingale (where again $X_{t}=X_{t}(x)$ and $\tau_{D}=\tau_{D}(x)$ ). By taking expectations we obtain

$$
h(x)-\mathbb{E}\left[h\left(X_{t \wedge \tau_{D}}\right)\right]=-\mathbb{E}[\int_{0}^{t \wedge \tau_{D}} \underbrace{L h\left(X_{s}\right)}_{\leq-1} d s] \geq \mathbb{E}\left[t \wedge \tau_{D}\right] .
$$

Hence,

$$
\begin{aligned}
\mathbb{E}\left[\tau_{D}\right] & =\mathbb{E}\left[\liminf _{t \rightarrow \infty} t \wedge \tau_{D}\right] \\
& \leq \liminf _{t \rightarrow \infty} \mathbb{E}\left[t \wedge \tau_{D}\right] \\
& \leq 2 \max _{y \in \bar{D}}|h(y)|<\infty
\end{aligned}
$$

which shows the claim.

Definition 3.9 (Generalized Dirichlet problem). Let $\varnothing \neq D \subsetneq M$ be an open and relatively compact domain and let $L$ be a second order PDO on $M$ as above. Assume to be given $g, k \in C(\bar{D}), k \geq 0$ and $\varphi \in C(\partial D)$. The generalized Dirichlet problem consists in finding $u \in C^{2}(D) \cap C(\bar{D})$ such that

$$
\left\{\begin{array}{l}
-L u+k u=g \quad \text { on } D \\
\left.u\right|_{\partial D}=\varphi
\end{array}\right.
$$

Theorem 3.10 (Stochastic representation of solutions to the GDP). Assume that $u$ solves $(\mathrm{GDP})$. For $x \in D$, let $X_{t}(x)$ be an L-diffusion, starting from $x$, and assume that

$$
\mathbb{E}\left[\tau_{D}(x)\right]<\infty \quad \text { for all } x \in D
$$

Then

$$
u(x)=\mathbb{E}\left[\varphi\left(X_{\tau_{D}}\right) \exp \left\{-\int_{0}^{\tau_{D}} k\left(X_{s}\right) d s\right\}+\int_{0}^{\tau_{D}} g\left(X_{s}\right) \exp \left\{-\int_{0}^{s} k\left(X_{r}\right) d r\right\} d s\right]
$$

where $\tau_{D}=\tau_{D}(x)$ and $X_{t}=X_{t}(x)$.

Proof. Consider the semimartingale

$$
N_{t}:=u\left(X_{t}\right) \exp \left\{-\int_{0}^{t} k\left(X_{s}\right) d s\right\}+\int_{0}^{t} g\left(X_{s}\right) \exp \left\{-\int_{0}^{s} k\left(X_{r}\right) d r\right\} d s .
$$

We find that

$$
\begin{aligned}
d N_{t} & =\exp \left\{-\int_{0}^{t} k\left(X_{s}\right) d s\right\}\left[d\left(u\left(X_{t}\right)\right)-u\left(X_{t}\right) k\left(X_{t}\right) d t+g\left(X_{t}\right) d t\right] \\
& \stackrel{m}{=} \exp \left\{-\int_{0}^{t} k\left(X_{s}\right) d s\right\}\left[(L u)\left(X_{t}\right) d t-u\left(X_{t}\right) k\left(X_{t}\right) d t+g\left(X_{t}\right) d t\right]=0,
\end{aligned}
$$

where as before the symbol $\stackrel{m}{=}$ denotes equality modulo differentials of (local) martingales. Thus, the process

$$
\left(N_{t \wedge \tau_{D}}\right)_{t \geq 0}
$$

is a martingale. In particular, by dominated convergence, we get

$$
u(x)=\mathbb{E}\left[N_{0}\right]=\mathbb{E}\left[N_{t \wedge \tau_{D}}\right] \rightarrow \mathbb{E}\left[N_{\tau_{D}}\right],
$$


and thus

$$
u(x)=\mathbb{E}\left[u\left(X_{\tau_{D}}\right) \exp \left\{-\int_{0}^{\tau_{D}} k\left(X_{s}\right) d s\right\}+\int_{0}^{\tau_{D}} g\left(X_{s}\right) \exp \left\{-\int_{0}^{s} k\left(X_{s}\right) d r\right\} d s\right] .
$$

Since $\left.u\right|_{\partial D}=\varphi$, we have $u\left(X_{\tau_{D}}\right)=\varphi\left(X_{\tau_{D}}\right)$ which gives the claim.

We shall consider the result of Theorem 3.10 in some special cases.

I. (Classical Feynman-Kac formula) Consider the boundary value problem of finding $u \in C^{2}(D) \cap C(\bar{D})$ such that

$$
\left\{\begin{array}{c}
-L u+k u=g \text { on } D \\
\left.u\right|_{\partial D}=0 .
\end{array}\right.
$$

Its solution is given by

$$
u(x)=\mathbb{E}\left[\int_{0}^{\tau_{D}(x)} g\left(X_{t}(x)\right) \exp \left\{-\int_{0}^{t} k\left(X_{r}(x)\right) d r\right\} d t\right], \quad x \in D .
$$

In particular, if $k \equiv 0$ then

$$
u(x)=\mathbb{E}\left[\int_{0}^{\tau_{D}(x)} g\left(X_{t}(x)\right) d t\right] \quad \text { (Green's kernel) }
$$

Note that $-L u=g$ is equivalent to $u=-L^{-1} g$. Thus the Green kernel gives an inverse to $-L$.

II. (Classical Dirichlet Problem) Consider the problem of finding $u \in C^{2}(D) \cap$ $C(\bar{D})$ such that

$$
\left\{\begin{array}{l}
L u=0 \text { on } D \\
\left.u\right|_{\partial D}=\varphi
\end{array}\right.
$$

If $X_{t}(x)$ is an $L$-diffusion, then

$$
u(x)=\mathbb{E}\left[\varphi\left(X_{\tau_{D}}(x)\right)\right]=\int_{\partial D} \varphi d \mu_{x}
$$

where the exit measure $\mu_{x}$ is given by

$$
\mu_{x}(B):=\mathbb{P}\left\{X_{\tau_{D}}(x) \in B\right\}, \quad B \subset \partial D \text { measurable. }
$$

Note that $u(x)=\int_{\partial D} \varphi d \mu(x)$ makes sense also for boundary functions $\varphi$ which are just bounded and measurable.

Example 3.11. Assume that $\partial D=A \cup B$ where $A \cap B=\varnothing$. In Physics a solution $u \in C^{2}(D) \cap C(\bar{D})$ to the Dirichlet problem

$$
\left\{\begin{array}{l}
L u=0 \text { on } D \\
u \mid A=1 \\
u \mid B=0
\end{array}\right.
$$

is called equilibrium potential for the capacitor $(A, B)$. Let $\left.\varphi\right|_{\partial D}$ be defined as

$$
\varphi(x)= \begin{cases}1, & \text { if } x \in A \\ 0, & \text { if } x \in B\end{cases}
$$

Then

$$
u(x)=\mathbb{E}\left[\varphi\left(X_{\tau_{D}}(x)\right)\right]=\mathbb{P}\left\{\tau_{A}(x)<\tau_{B}(x)\right\}
$$


where

$$
\begin{aligned}
& \tau_{A}(x)=\inf \left\{t>0, X_{t}(x) \in A\right\}, \\
& \tau_{B}(x)=\inf \left\{t>0, X_{t}(x) \in B\right\} .
\end{aligned}
$$

Thus $u(x)$ corresponds to the probability that an L-diffusion, starting from $x$, hits $A$ before hitting $B$.

3.3. Parabolic boundary value problems. Let $D \subset M$ be an open and relatively compact domain. Consider a second order PDO $L$ on $M$ and let $\left(X_{t}(x)\right)_{t \geq 0}$ be an $L$-diffusion. Let $T>0$ and $V$ be a measurable function on $D$ such that

$$
\mathbb{E}\left[\exp \left(\int_{0}^{T \wedge \tau_{D}(x)} V_{-}\left(X_{s}(x)\right) d s\right)\right]<\infty, \quad \forall x \in D,
$$

where $V_{-}:=(-V) \vee 0$ denotes the negative part of $V$ and $\tau_{D}(x)=\inf \{s \geq 0$ : $\left.X_{t}(x) \in \partial D\right\}$. Furthermore, let $f, g \in C(\bar{D})$ and $\varphi \in C(\partial D)$.

Problem. Find a solution to the following parabolic boundary value problem:

$$
\left\{\begin{array}{l}
\frac{\partial}{\partial t} u=L u-V u+g \text { on }[0, T] \times D \\
\left.u(t, \cdot)\right|_{\partial D}=\varphi \text { for } t \in[0, T] \\
\left.u\right|_{t=0}=f
\end{array}\right.
$$

Note that necessarily $\left.f\right|_{\partial D}=\varphi$.

Theorem 3.12. Every solution $u \in C^{2}([0, T] \times D) \cap C([0, T] \times \bar{D})$ of $(\mathrm{BVP})$ is of the form

$u(t, x)=\mathbb{E}\left[f\left(X_{t \wedge \tau_{D}}\right) \exp \left(-\int_{0}^{t \wedge \tau_{D}} V\left(X_{s}\right) d s\right)+\int_{0}^{t \wedge \tau_{D}} g\left(X_{s}\right) \exp \left(-\int_{0}^{s} V\left(X_{r}\right) d r\right) d s\right]$,

where $X_{t}=X_{t}(x)$ and $\tau_{D}=\tau_{D}(x)$.

Proof. For $0<t_{0} \leq T$, we check by Itô's formula that

$N_{t}:=u\left(t_{0}-t, X_{t}\right) \exp \left(-\int_{0}^{t} V\left(X_{s}\right) d s\right)+\int_{0}^{t} g\left(X_{s}\right) \exp \left(-\int_{0}^{s} V\left(X_{r}\right) d r\right) d s, \quad t \leq t_{0} \wedge \tau_{D}$,

is a martingale. Then it suffices to evaluate $u\left(t_{0}, x\right)=\mathbb{E}\left[N_{0}\right]=\mathbb{E}\left[N_{t_{0} \wedge \tau_{D}}\right]$ which gives the claim.

In the discussion of this section we restricted ourselves to representation formulas for solutions to elliptic-parabolic equations of second order. For establishing existence of solutions by probabilistic methods the reader may consult [54].

\section{Stochastic Calculus on manifolds}

4.1. Quadratic variation and integration of 1-forms. In this section we give canonical constructions related to continuous semimartingales on a manifold $M$, including the quadratic variation of continuous semimartingales with respect to bilinear forms on $T M$ and the integral of 1-forms on $M$ along semimartingales, see [19] for more details.

The following technical lemma on continuous processes is well-known (e.g. [24]) and very useful for a localization in space of continuous adapted processes, besides the usual localization in time (through a sequence of stopping times). 
Lemma 4.1. Let $\left(V_{k}\right)_{k \in \mathbb{N}}$ be a countable covering of $M$ by open sets $V_{k}$ and $X$ be a continuous adapted $M$-valued process. Then there exists a non-decreasing sequence $\left(\tau_{n}\right)_{n \geq 0}$ of stopping times with $\tau_{0}=0$ and $\sup _{n} \tau_{n}=\infty$, such that on each of the intervals $\left[\tau_{n}, \tau_{n+1}\right] \cap\left(\mathbb{R}_{+} \times\left\{\tau_{n}<\tau_{n+1}\right\}\right)$ the process $X$ takes values only in one of the $V_{k}$.

Given a filtered probability space $\left(\Omega, \mathscr{F}, \mathbb{P} ;\left(\mathscr{F}_{t}\right)_{t \in \mathbb{R}_{+}}\right)$we denote by $\mathscr{S}$ the vector space of real-valued continuous semimartingales:

$$
\mathscr{S}=\mathscr{M}_{0} \oplus \mathscr{A}
$$

where $\mathscr{M}_{0}$ denotes the space of continuous local martingales starting at 0 and $\mathscr{A}$ the space of continuous adapted processes pathwise locally of bounded variation.

Lemma 4.2. Let $M$ be an arbitrary differentiable manifold. There exist finitely many functions $h^{1}, \ldots, h^{\ell} \in C^{\infty}(M)$ such that the following properties hold:

(i) Each function $f \in C^{\infty}(M)$ factorizes through $\left(h^{1}, \ldots, h^{\ell}\right)$ as $f=\bar{f} \circ\left(h^{1}, \ldots, h^{\ell}\right)$ for some $\bar{f} \in C^{\infty}\left(\mathbb{R}^{\ell}\right)$.

(ii) Each section $b \in \Gamma\left(T^{*} M \otimes T^{*} M\right)$ can be written as $b=\sum_{i, j=1}^{\ell} b_{i j} d h^{i} \otimes d h^{j}$ with functions $b_{i j} \in C^{\infty}(M)$.

(iii) Each differential form $\alpha \in \Gamma\left(T^{*} M\right)$ can be written as $\alpha=\sum_{i=1}^{\ell} \alpha_{i} d h^{i}$ with functions $\alpha_{i} \in C^{\infty}(M)$.

Proof. We represent $M$ via a Whitney embedding $h: M \hookrightarrow \mathbb{R}^{\ell}$ as a closed submanifold of some $\mathbb{R}^{\ell}$. Then there exists a differentiable partition $\left(\varphi_{\lambda}\right)_{\lambda \in \Lambda}$ of the unity on $M$ and a family $\left(I_{\lambda}\right)_{\lambda \in \Lambda}$ of subsets $I_{\lambda} \subset\{1, \ldots, \ell\}$ with the following property: for each $\lambda \in \Lambda$ the $\left(h^{i}\right)_{i \in I_{\lambda}}$ define a chart for $M$ on some open neighbourhood of $\operatorname{supp}\left(\varphi_{\lambda}\right)$.

Part (i) is evident: One defines $\bar{f} \mid h(M)$ through $f=\bar{f} \circ h$ and extends $\bar{f}$ constantly along the normal subspaces $\perp_{x} M$ to an open neighbourhood of $M \cong h(M)$. Then, one may smoothen $\bar{f}$ by multiplication with a function identical 1 locally about $h(M)$ and vanishing outside a suitable larger neighbourhood. To part (ii): Note that $\varphi_{\lambda} b=\sum_{i, j=1}^{\ell} b_{i j}^{\lambda} d h^{i} \otimes d h^{j}$ with $b_{i j}^{\lambda} \in C^{\infty}(M)$ such that $\operatorname{supp}\left(b_{i j}^{\lambda}\right) \subset$ $\operatorname{supp}\left(\varphi_{\lambda}\right)$ and $b_{i j}^{\lambda}:=0$ for $\{i, j\} \not \subset I_{\lambda}$, but then

$$
b=\sum_{i, j=1}^{\ell} b_{i j} d h^{i} \otimes d h^{j} \quad \text { where } b_{i j}:=\sum_{\lambda} b_{i j}^{\lambda} .
$$

The proof of part (iii) is analogous to (ii).

Theorem 4.3. Let $X$ be an $M$-valued semimartingale. There exists a unique linear mapping

$$
\Gamma\left(T^{*} M \otimes T^{*} M\right) \rightarrow \mathscr{A}, \quad b \mapsto \int b(d X, d X),
$$

such that for all $f, g \in C^{\infty}(M)$,

$$
\begin{aligned}
& d f \otimes d g \mapsto[f(X), g(X)] \\
& f b \mapsto \int f(X) b(d X, d X) .
\end{aligned}
$$

Here, by definition $b(d X, d X):=d \int b(d X, d X)$. Recall that $[f(X), g(X)]$ in condition (4.1) denotes the quadratic covariation process of $f(X)$ and $g(X)$.

Definition 4.4. The process $\int b(d X, d X)$ is called integral of $b$ along $X$ or $b$ quadratic variation of $X$. The random variable giving its value at time $t$ is usually written as $\int_{0}^{t} b(d X, d X)$. 
Proof (of Theorem 4.3). By Lemma 4.2 (ii) each section $b \in \Gamma\left(T^{*} M \otimes T^{*} M\right)$ can be represented as $b=\sum b_{i j} d h^{i} \otimes d h^{j}$. We define

$$
\int b(d X, d X):=\sum \int\left(b_{i j} \circ X\right) d\left[h^{i}(X), h^{j}(X)\right] .
$$

Then uniqueness is obvious; to prove existence it remains to show that (4.3) is well-defined. To this end assume that

$$
b=\sum_{\text {finite }} u_{\nu} d f^{\nu} \otimes d g^{\nu}=0 .
$$

We need to check that

$$
\sum_{\nu} u_{\nu}(X) d\left[f^{\nu}(X), g^{\nu}(X)\right]=0
$$

as well. Without loss of generality, by means of Lemma 4.1, we may assume that $h$ is already a global chart for $M$. According to Lemma 4.2 (i) we write $u_{\nu}=\bar{u}_{\nu} \circ h$, $f^{\nu}=\bar{f}^{\nu} \circ h$ and $g^{\nu}=\bar{g}^{\nu} \circ h$ in terms of appropriate extensions $\bar{u}_{\nu}, \bar{f}^{\nu}, \bar{g}^{\nu} \in C^{\infty}\left(\mathbb{R}^{\ell}\right)$. Defining $\bar{X}=h \circ X$, the claim then follows from the following calculation:

$$
\begin{aligned}
\sum_{\nu} & u_{\nu}(X) d\left[f^{\nu}(X), g^{\nu}(X)\right]=\sum_{\nu} \bar{u}_{\nu}(\bar{X}) d\left[\bar{f}^{\nu}(\bar{X}), \bar{g}^{\nu}(\bar{X})\right] \\
& =\sum_{i, j} \sum_{\nu} \bar{u}_{\nu}(\bar{X})\left(D_{i} \bar{f}^{\nu}\right)(\bar{X})\left(D_{j} \bar{g}^{\nu}\right)(\bar{X}) d\left[\bar{X}^{i}, \bar{X}^{j}\right] \\
& =\sum_{i, j}\left(\sum_{\nu} u_{\nu} d f^{\nu} \otimes d g^{\nu}\right)\left(\left(\frac{\partial}{\partial h^{i}}\right)_{X},\left(\frac{\partial}{\partial h^{j}}\right)_{X}\right) d\left[\bar{X}^{i}, \bar{X}^{j}\right]=0 .
\end{aligned}
$$

Corollary 4.5. The b-quadratic variation $\int b(d X, d X)$ depends only on the symmetric part of $b$. In particular, $\int b(d X, d X)=0$ if $b$ is antisymmetric.

Proof. Defining $\bar{b}(A, B):=b(B, A)$, the assignment $b \mapsto \int \bar{b}(d X, d X)$ has the defining properties (4.1) and (4.2) as well.

Theorem 4.6 (Pullback formula for the $b$-quadratic variation). Let $\phi: M \rightarrow N$ be a differentiable map and $b \in \Gamma\left(T^{*} N \otimes T^{*} N\right)$. Let $\phi^{*} b \in \Gamma\left(T^{*} M \otimes T^{*} M\right)$ be the pullback of $b$ via $\phi$, i.e

$$
\left(\phi^{*} b\right)_{p}(u, v):=b_{\phi(p)}\left(d \phi_{p} u, d \phi_{p} v\right), \quad u, v \in T_{p} M, p \in M .
$$

Then, for any semimartingale $X$ on $M$,

$$
\int\left(\phi^{*} b\right)(d X, d X)=\int b(d(\phi \circ X), d(\phi \circ X)) .
$$

Proof. The left-hand side of (4.4) obviously has the defining properties for the $b$-quadratic variation of the image process $\phi \circ X$.

We now turn to the problem of integrating 1-forms on $M$ along $M$-valued semimartingales.

Theorem 4.7. Let $X$ be a semimartingale taking values in $M$. There is a unique linear mapping

$$
\Gamma\left(T^{*} M\right) \rightarrow \mathscr{S}, \quad \alpha \mapsto \int \alpha(\circ d X) \equiv \int_{X} \alpha,
$$

such that for all $f \in C^{\infty}(M)$,

$$
\begin{aligned}
d f & \mapsto f(X)-f\left(X_{0}\right) \\
f \alpha & \mapsto \int f(X) \circ \alpha(\circ d X) .
\end{aligned}
$$


In (4.6) the integral means the Stratonovich integral of the process $f(X)$ with respect to the semimartingale $\int \alpha(\circ d X)$. Thus, in other words, $f(X) \circ \alpha(\circ d X) \equiv f(X) \circ$ $d\left(\int \alpha(\circ d X)\right)$.

Definition 4.8 (Stratonovich integral of 1-forms along semimartingales). The process $\int \alpha(\circ d X)$ is called the Stratonovich integral of $\alpha$ along $X$. We also write $\int_{X} \alpha$ instead of $\int \alpha(\circ d X)$.

Proof (of Theorem 4.7). By Lemma 4.2 (iii) each differential form $\alpha \in \Gamma\left(T^{*} M\right)$ can be represented as $\alpha=\sum_{i} \alpha_{i} d h^{i}$ with functions $\alpha_{i} \in C^{\infty}(M)$. We define

$$
\int_{X} \alpha:=\sum_{i} \int \alpha_{i}(X) \circ d\left(h^{i}(X)\right) .
$$

Uniqueness is again obvious; it is thus sufficient to show that formula (4.7) is welldefined. To this end, we have to verify that if $\alpha=\sum_{\text {finite }} u_{\nu} d f^{\nu}=0$ then

$$
\sum_{\nu} u_{\nu}(X) \circ d\left(f^{\nu}(X)\right)=0
$$

holds as well. Proceeding as in the proof of Theorem 4.7, without loss of generality, we may assume again that $h$ is already a global chart for $M$. But then we have

$$
\begin{aligned}
\sum_{\nu} u_{\nu}(X) \circ d\left(f^{\nu}(X)\right) & =\sum_{\nu} \bar{u}_{\nu}(\bar{X}) \circ d\left(\bar{f}^{\nu}(\bar{X})\right) \\
& =\sum_{i} \sum_{\nu} \bar{u}_{\nu}(\bar{X}) \circ\left[D_{i} \bar{f}^{\nu}(\bar{X}) \circ d \bar{X}^{i}\right] \\
& =\sum_{i}\left(\left(\sum_{\nu} u_{\nu} d f^{\nu}\right)\left(\frac{\partial}{\partial h^{i}}\right)_{X}\right) \circ d \bar{X}^{i}=0
\end{aligned}
$$

which gives the claim.

Example 4.9. In the special case of a deterministic $C^{1}$ curve $X$ in $M$, say $X_{t}=$ $x(t)$, which is trivially a semimartingale, we obtain

$$
\int_{X} \alpha=\int \alpha(\dot{x}(t)) d t, \quad \alpha \in \Gamma\left(T^{*} M\right) .
$$

Indeed, the right-hand side of (4.8) obviously has the defining properties of $\int_{X} \alpha$.

Theorem 4.10 (Pullback formula for the Stratonovich integral of a 1-form). Let $\phi: M \rightarrow N$ be a differentiable map and $\alpha \in \Gamma\left(T^{*} N\right)$. Then, for any semimartingale $X$ on $M$, we have

$$
\int_{X} \phi^{*} \alpha=\int_{\phi \circ X} \alpha
$$

Proof. The left-hand side of Eq. (4.9) satisfies the defining properties for the Stratonovich integral of $\alpha$ along $\phi \circ X$. By uniqueness we therefore have equality.

Remark 4.11. Let $\alpha, \beta \in \Gamma\left(T^{*} M\right)$. Then $\alpha \otimes \beta \in \Gamma\left(T^{*} M \otimes T^{*} M\right)$ and for the quadratic covariation process of $\int_{X} \alpha$ and $\int_{X} \beta$ we have the formula:

$$
\left[\int_{X} \alpha, \int_{X} \beta\right]=\int(\alpha \otimes \beta)(d X, d X)
$$


4.2. Martingales and Brownian motions. The aim of this section is to introduce martingales and Brownian motions on manifolds. This task requires additional geometric structures on the manifolds: linear connections and Riemannian metrics. These results will then be extended later on to the setting of sub-Riemannian geometry where the metric is only defined on a subbundle of $T M$.

Notation 4.12. Let $\pi: T M \rightarrow M$ be the tangent bundle over $M$. A linear connection in $T M$, or equivalently a covariant derivative on $T M$, is a $\mathbb{R}$-linear mapping

$$
\nabla: \Gamma(T M) \rightarrow \Gamma\left(T^{*} M \otimes T M\right)
$$

satisfying the product rule $\nabla(f X)=d f \otimes X+f \nabla X$ for all $X \in \Gamma(T M)$ and $f \in C^{\infty}(M)$. Alternatively, (4.10) may be written as a mapping

$$
\Gamma(T M) \times \Gamma(T M) \rightarrow \Gamma(T M), \quad(A, X) \mapsto \nabla_{A} X \equiv(\nabla X) A
$$

which is $C^{\infty}(M)$-linear in the first variable and derivative in the second variable. For $f \in C^{\infty}(M)$, we have the second fundamental form (or Hessian) of $f$ defined as

$$
\nabla d f \equiv \operatorname{Hess} f \in \Gamma\left(T^{*} M \otimes T^{*} M\right), \quad(\nabla d f)(A, B)=A B f-\left(\nabla_{A} B\right) f .
$$

The bilinear form

$$
(A, B) \mapsto(\nabla d f)(A, B),
$$

is symmetric for each $f \in C^{\infty}(M)$ if and only if the connection $\nabla$ is torsion-free, i.e. if for all $A, B \in \Gamma(T M)$,

$$
T(A, B) \equiv \nabla_{A} B-\nabla_{B} A-[A, B]=0 .
$$

Definition 4.13 ( $\nabla$-martingale). Let $M$ be a manifold and $\nabla$ be a linear connection in $T M$. An $M$-valued semimartingale $X$ defined on some filtered probability space $\left(\Omega, \mathscr{F}, \mathbb{P} ;\left(\mathscr{F}_{t}\right)_{t \geq 0}\right)$, is called $\nabla$-martingale if for each $f \in C^{\infty}(M)$ :

$$
d(f \circ X)-\frac{1}{2}(\nabla d f)(d X, d X) \stackrel{\underline{m}}{=} 0
$$

where $\stackrel{m}{=}$ means equality modulo differentials of local martingales.

Since $(\nabla d f)(d X, d X)$ only depends on the symmetric part of $\nabla d f$, one may always assume that the linear connection $\nabla$ is torsion-free. Symmetrization of the connection does not change the class of $\nabla$-martingales.

Example 4.14. In the special case of $M=\mathbb{R}^{n}$ equipped with the canonical linear connection $\nabla_{D_{i}} D_{j}=0$, we have

$$
(\nabla d f)\left(D_{i}, D_{j}\right)=D_{i} D_{j} f
$$

and hence $\nabla$-martingales in the sense of Definition 4.13 coincide with the usual class of continuous local martingales on $\mathbb{R}^{n}$. Indeed, according to Itô's formula, a continuous $\mathbb{R}^{n}$-valued semimartingale $X$ is a local martingale if and only if

$$
d(f \circ X)-\frac{1}{2} \sum_{i, j}\left(D_{i} D_{j} f\right)(X) d\left[X^{i}, X^{j}\right] \stackrel{\mathrm{m}}{=} 0
$$

for all $f \in C^{\infty}\left(\mathbb{R}^{n}\right)$. This is exactly condition (4.11) of Definition 4.13.

Remark 4.15 (Martingales as solutions of SDEs). Let $\nabla$ be a linear connection on $T M$ which without loss of generality is torsion-free. Let $A_{0} \in \Gamma(T M)$ and $A \in \Gamma\left(\operatorname{Hom}\left(M \times \mathbb{R}^{r}, T M\right)\right)$ and suppose that $X$ is solution to the $\mathrm{SDE}$

$$
d X=A_{0}(X) d t+A(X) \circ d Z .
$$


Here $Z$ may be an arbitrary continuous $\mathbb{R}^{r}$-valued semimartingale. Then for $f \in$ $C^{\infty}(M)$ we have

$$
d(f \circ X)=\left(A_{0} f\right)(X) d t+\sum_{i=1}^{r}\left(A_{i} f\right)(X) d Z^{i}+\frac{1}{2} \sum_{i, j=1}^{r}\left(A_{i} A_{j} f\right)(X) d\left[Z^{i}, Z^{j}\right] .
$$

where $A_{i}=A(\cdot) e_{i} \in \Gamma(T M)$ for $i=1, \ldots, r$. Since $(\nabla d f)\left(A_{i}, A_{j}\right)=A_{i} A_{j} f-$ $\left(\nabla_{A_{i}} A_{j}\right) f$ and since on the other hand

$$
(\nabla d f)(d X, d X)=\sum_{i, j=1}^{r}(\nabla d f)\left(A_{i}, A_{j}\right)(X) d\left[Z^{i}, Z^{j}\right]
$$

we obtain

$$
\begin{aligned}
& d(f \circ X)-\frac{1}{2}(\nabla d f)(d X, d X) \\
& \quad=\left(A_{0} f\right)(X) d t+\sum_{i=1}^{r}\left(A_{i} f\right)(X) d Z^{i}+\frac{1}{2} \sum_{i, j=1}^{r}\left(\nabla_{A_{i}} A_{j} f\right)(X) d\left[Z^{i}, Z^{j}\right] .
\end{aligned}
$$

Denoting the drift of the semimartingale $Z$ by $Z^{\text {drift }}$, we obtain that $X$ is a $\nabla$ martingale if

$$
\left(A_{0} f\right)(X) d t+\sum_{i=1}^{r}\left(A_{i} f\right)(X) d\left(Z^{\mathrm{drift}}\right)^{i}+\frac{1}{2} \sum_{i, j=1}^{r}\left(\nabla_{A_{i}} A_{j} f\right)(X) d\left[Z^{i}, Z^{j}\right]=0
$$

for any $f \in C^{\infty}(M)$. In the special case when $Z$ is a Brownian motion on $\mathbb{R}^{r}$ we find that solutions $X$ to the SDE (4.12) are a $\nabla$-martingales if

$$
A_{0}=-\frac{1}{2} \sum_{i=1}^{r} \nabla_{A_{i}} A_{i}
$$

Definition 4.16 (Riemannian quadratic variation). Let $(M, g)=(M,\langle\cdot, \cdot\rangle)$ be a Riemannian manifold and $X$ be a semimartingale taking values in $M$. The process

$$
[X, X]:=\int g(d X, d X)=\int\langle d X, d X\rangle
$$

is called Riemannian quadratic variation of $X$.

Theorem 4.17 (Lévy's characterization of $M$-valued Brownian motions). Let $(M, g)$ be a Riemannian manifold and $\nabla$ be the Levi-Civita connection. For a semimartingale $X$ of maximal lifetime and taking values in $M$, the following conditions are equivalent:

(i) $X$ is a Brownian motion on $(M, g)$, i.e. for any $f \in C^{\infty}(M)$ the real-valued process

$$
f \circ X-\frac{1}{2} \int \Delta f \circ X d t
$$

is a local martingale; here $\Delta f=$ trace $\nabla d f \in C^{\infty}(M)$ denotes the LaplaceBeltrami operator on $M$.

(ii) $X$ is a $\nabla$-martingale such that

$$
[f(X), f(X)]=\int\|\nabla f\|^{2}(X) d t
$$

for every $f \in C^{\infty}(M)$.

(iii) $X$ is a $\nabla$-martingale such that

$$
\int b(d X, d X)=\int(\operatorname{trace} b)(X) d t
$$

for every $b \in \Gamma\left(T^{*} M \otimes T^{*} M\right)$. 
In particular, for the Riemannian quadratic variation (4.13) of $X$, we then have

$$
\int_{0}^{t} g(d X, d X)=t \operatorname{dim} M
$$

Proof. 1) To prove (ii) $\Longleftrightarrow$ (iii) we verify that for $X$ the following two conditions are equivalent:

(a) $[f(X), f(X)]=\int\|\nabla f\|^{2}(X) d t$

(b) $\int b(d X, d X)=\int($ trace $b)(X) d t$ for every $b \in \Gamma\left(T^{*} M \otimes T^{*} M\right)$.

Indeed, for $f, h \in C^{\infty}(M)$ we have

$$
\begin{aligned}
\operatorname{trace}(d f \otimes d h) & =\sum_{i}(d f \otimes d h)\left(e_{i}, e_{i}\right)=\sum_{i}(d f)\left(e_{i}\right)(d h)\left(e_{i}\right) \\
& =\sum_{i}\left\langle\nabla f, e_{i}\right\rangle\left\langle\nabla h, e_{i}\right\rangle=\langle\nabla f, \nabla h\rangle .
\end{aligned}
$$

The implication (b) $\Rightarrow(\mathrm{a})$ is then the special case for $b=d f \otimes d f$. To verify the direction $(\mathrm{a}) \Rightarrow(\mathrm{b})$, first note that (a) implies by polarization

$$
[f(X), h(X)]=\int\langle\nabla f \circ X, \nabla h \circ X\rangle d t
$$

for $f, h \in C^{\infty}(M)$. Thus $[f \circ X, h \circ X]=\int(d f \otimes d h)(d X, d X)=\int \operatorname{trace}(d f \otimes$ $d h)(X) d t$. By means of the uniqueness part of Theorem 4.3, we get

$$
\int b(d X, d X)=\int(\operatorname{trace} b)(X) d t
$$

for any bilinear form $b \in \Gamma\left(T^{*} M \otimes T^{*} M\right)$.

2) (iii) $\Rightarrow$ (i): Part 1 applied to the given $\nabla$-martingale $X$ shows that $b(d X, d X)=$ (trace $b)(X) d t$ for bilinear forms $b \in \Gamma\left(T^{*} M \otimes T^{*} M\right)$; thus in particular for $b=\nabla d f$,

$$
d(f \circ X) \stackrel{\mathrm{m}}{=} \frac{1}{2} \nabla d f(d X, d X)=\frac{1}{2}(\Delta f)(X) d t .
$$

3) (i) $\Rightarrow$ (ii): Now let $X$ be a Brownian motion on $M$. According to $\nabla d f^{2}=$ $2(f \nabla d f+d f \otimes d f)$ we first note that $\Delta\left(f^{2}\right)=2 f \Delta f+2\|\nabla f\|^{2}$, and thus

$$
d\left(f^{2} \circ X\right) \stackrel{\mathrm{m}}{=} \frac{1}{2}\left(\Delta f^{2}\right)(X) d t=(f \Delta f)(X) d t+\|\nabla f\|^{2}(X) d t .
$$

On the other hand, by means of Itô's formula,

$d\left(f^{2} \circ X\right)=2 f(X) d(f \circ X)+d[f(X), f(X)] \stackrel{\mathrm{m}}{=} f(X)(\Delta f)(X) d t+d[f(X), f(X)]$.

Uniqueness of the Doob-Meyer decomposition implies

$$
[f(X), f(X)]=\int\|\nabla f\|^{2}(X) d t .
$$

Finally, once again by means of part 1, the last formula gives

$$
\nabla d f(d X, d X)=(\operatorname{trace} \nabla d f)(X) d t=(\Delta f)(X) d t
$$

from where we conclude that $X$ is a $\nabla$-martingale.

On $\mathbb{R}^{n}$ with the canonical Euclidean metric, Brownian motions in the sense of Lévy's characterization coincide with the usual class of $\mathbb{R}^{n}$-valued Brownian motions.

Theorem 4.18 ( $M$-valued Brownian motions as solutions of an SDE). Let $(M, g)$ be a Riemannian manifold and $\nabla$ be the Levi-Civita connection on $M$. Consider the SDE

$$
d X=A_{0}(X) d t+A(X) \circ d B
$$


with $A_{0} \in \Gamma(T M)$ and $A \in \Gamma\left(\operatorname{Hom}\left(M \times \mathbb{R}^{r}, T M\right)\right)$; here $B$ a Brownian motion on $\mathbb{R}^{r}$. Then maximal solutions to (4.14) are Brownian motions on $(M, g)$ if the two subsequent conditions are satisfied:

(i) $A_{0}=-\frac{1}{2} \sum_{i} \nabla_{A_{i}} A_{i}$ with $A_{i} \equiv A(\cdot) e_{i}$ for $i=1, \ldots, r$.

(ii) The map $A(x)^{*}: T_{x} M \rightarrow \mathbb{R}^{r}$ is an isometric embedding for every $x \in M$, i.e., $A(x) A(x)^{*}=\operatorname{id}_{T_{x} M}$ where $A(x)^{*}$ is the adjoint to $A(x) \in \operatorname{Hom}\left(\mathbb{R}^{r}, T_{x} M\right)$.

Proof. Let $X$ be a solution to Eq. (4.14) and assume that conditions (i) and (ii) are satisfied. According to Remark 4.15 condition (i) guarantees that $X$ is a $\nabla$ martingale. In addition, we have for $f \in C^{\infty}(M)$,

$$
d(f \circ X) \stackrel{\mathrm{m}}{=} \frac{1}{2} \sum_{i=1}^{r}(\nabla d f)\left(A_{i}, A_{i}\right)(X) d t .
$$

It is thus sufficient to verify that

$$
\sum_{i}(\nabla d f)\left(A_{i}, A_{i}\right)=\Delta f .
$$

This is however a straight-forward consequence of condition (ii).

Remark 4.19. The conditions (i) and (ii) of Theorem 4.18 can always be satisfied for $r$ sufficiently large. For instance, let $M \hookrightarrow \mathbb{R}^{r}$ be a Whitney embedding. Then $T_{x} M$ can be seen as a subspace $\mathbb{R}^{r}$ for each $x \in M$. Defining $A \in \Gamma\left(\operatorname{Hom}\left(M \times \mathbb{R}^{r}, T M\right)\right)$ fibrewise as orthogonal projection $A(x): \mathbb{R}^{r} \rightarrow T_{x} M$ onto $T_{x} M$ and setting $A_{0}=-\frac{1}{2} \sum_{i} \nabla_{A_{i}} A_{i}$, then every solution to the SDE (4.14) (with a given initial condition) is a Brownian motion on $(M, g)$. The drawback of this construction is that to a given Riemannian manifold $(M, g)$ there is no canonical choice of the coefficients $A_{0}$ and $A$; there is however a canonical SDE on the orthonormal frame bundle $\mathrm{O}(T M)$ over $M$ such that its solutions project to Brownian motions on $(M, g)$. We deal with this construction in the next subsection.

Theorem 4.20 (Brownian motions on submanifolds of $\mathbb{R}^{n}$ ). Let $M$ be a submanifold of $\mathbb{R}^{n}$ endowed with the induced Riemannian metric. Consider the SDE

$$
d X=A(X) \circ d B
$$

where $B$ is a Brownian motion on $\mathbb{R}^{n}$ and

$$
A \in \Gamma\left(\operatorname{Hom}\left(M \times \mathbb{R}^{n}, T M\right)\right), \quad(x, v) \mapsto A(x) v,
$$

such that $A(x): \mathbb{R}^{n} \rightarrow T_{x} M$ is the orthogonal projection onto $T_{x} M$. Then every solution of (4.15), to some specified initial condition, gives a Brownian motion on $(M, g)$.

Proof. In terms of the vector fields $A_{i} \equiv A(\cdot) e_{i} \in \Gamma(T M), i=1, \ldots, n$, it is sufficient by Theorem 4.18 to verify that $\sum_{i} \nabla_{A_{i}} A_{i}=0$. This is however a straightforward calculation.

4.3. Parallel transport and stochastically moving frames. The fundamental observation that diffusion processes on a manifold $M$ can be horizontally lifted via a connection to the frame bundle over $M$ goes back to the pioneering work of Malliavin, Eells and Elworthy. Conversely, solving SDEs on the frame bundle and projecting the solution down to the manifold $M$ allows canonical constructions of diffusion processes on $M$.

Intuitively this procedure corresponds to a "rolling without slipping" of the manifold along the trajectories of a continuous $\mathbb{R}^{n}$-valued semimartingale. It allows to construct to each semimartingale in $T_{x} M$ its stochastic development on $M$, together with a notion of parallel transport along the paths of the obtained process. Clearly 
this method requires a connection on $M$. The problem that in sub-Riemannian geometry typically only "partial connections" are canonically given will be addressed in the next subsection.

Notation 4.21. Let $M$ be an $n$-dimensional differentiable manifold and denote by $P=\mathrm{L}(T M)$ its frame bundle. Then $\pi: P \rightarrow M$ is a $G$-principal bundle with $G=\mathrm{GL}(n ; \mathbb{R})$. The fibre $P_{x}$ consists of the linear isomorphisms $u: \mathbb{R}^{n} \rightarrow T_{x} M$ where $u \in P_{x}$ is identified with the $\mathbb{R}$-basis

$$
\left(u_{1}, \ldots, u_{n}\right):=\left(u e_{1}, \ldots, u e_{n}\right) .
$$

A linear connection in $T M$ induces canonically a $G$-connection in $P$ given as a $G$ invariant differentiable splitting $h$ of the following exact sequence of vector bundles over $P$ :

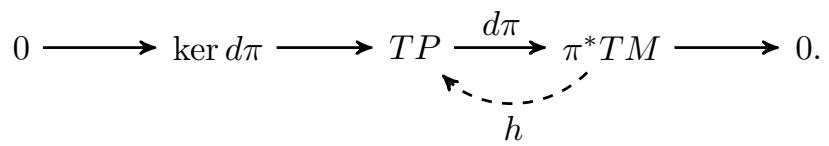

This splitting induces a decomposition of $T P$ :

$$
T P=V \oplus H:=\operatorname{ker} d \pi \oplus h\left(\pi^{*} T M\right) .
$$

$G$-invariance of the splitting means that $H_{u g}=\left(d R_{g}\right) H_{u}$ for each $u \in P$, where $R_{g} u:=u g$ denotes the right action of $g \in G$. For $u \in P$, we call $H_{u}$ the horizontal space at $u$ and $V_{u}=\left\{v \in T_{u} P:(d \pi) v=0\right\}$ the vertical space at $u$. The bundle isomorphism

$$
h: \pi^{*} T M \stackrel{\sim}{\longrightarrow} \hookrightarrow T P
$$

is called horizontal lift of the $G$-connection; fibrewise it reads as $h_{u}: T_{\pi(u)} M \stackrel{\sim}{\longrightarrow}$ $H_{u}$.

By means of the $G$-connection in $P$ each vector field $X \in \Gamma(T P)$ decomposes in a horizontal and a vertical part:

$$
X=\operatorname{hor} X+\operatorname{vert} X .
$$

Definition 4.22 (Connection form). Each $u \in P$ defines an embedding

$$
I_{u}: G \hookrightarrow P, \quad g \mapsto u g .
$$

Its differential at the unit element $e \in G$,

$$
\iota_{u} \equiv\left(d I_{u}\right)_{e}: T_{e} G \rightarrow T_{u} P, \quad A \longmapsto \hat{A}(u),
$$

gives an identification $\kappa_{u}: \mathfrak{g} \stackrel{\sim}{\longrightarrow} V_{u}$ of the Lie algebra $\mathfrak{g}=T_{e} G$ of $G$ with the vertical fibre $V_{u}$ at $u$. The vertical vector field $\hat{A} \in \Gamma(T P)$ on $P$ defined by (4.17) is called standard-vertical vector field to $A \in \mathfrak{g}$. The $\mathfrak{g}$-valued 1 -form $\omega \in \Gamma\left(T^{*} P \otimes \mathfrak{g}\right)$ on $P$ defined by

$$
\omega_{u}\left(X_{u}\right):=\kappa_{u}^{-1}(\operatorname{vert} X)_{u}, \quad X \in \Gamma(T P),
$$

is called connection form of the $G$-connection.

Note that for the frame bundle $\pi: \mathrm{L}(T M) \rightarrow M$ over $M$ we have $\mathfrak{g}=\mathrm{GL}(n ; \mathbb{R})$. In case that $M$ is a Riemannian manifold it is natural to consider the orthonormal frame bundle $\pi: \mathrm{O}(T M) \rightarrow M$ over $M$ with structure group $G=\mathrm{O}(n ; \mathbb{R})$. The fibre $P_{x}$ then consists of the linear isometries $u: \mathbb{R}^{n} \rightarrow T_{x} M$. As above a metric connection on $T M$ then gives rise to a $G$-invariant splitting $T P=V \oplus H$. The connection form then takes its values in the Lie algebra $\mathfrak{g}$ of skew symmetric $n \times n$ matrices. 
In the sequel we deal with the two cases of $G$-principal bundles: $P=\mathrm{L}(T M)$ over a manifold $M$ with $G=\mathrm{GL}(n ; \mathbb{R})$ and $P=\mathrm{O}(T M)$ over a Riemannian manifold $M$ with $G=\mathrm{O}(n ; \mathbb{R})$. In addition to the $\mathfrak{g}$-valued connection form (see Definition 4.22) we have the canonical 1-form

$$
\vartheta \in \Gamma\left(T^{*} P \otimes \mathbb{R}^{n}\right), \quad \vartheta_{u}\left(X_{u}\right):=u^{-1}\left(d \pi X_{u}\right), u \in P \text { and } X \in \Gamma(T P),
$$

where as usual we read $u \in P$ as linear isomorphism, resp. isometry, $u: \mathbb{R}^{n} \stackrel{\sim}{\longrightarrow}$ $T_{\pi(u)} M$.

Remark 4.23. The frame bundles $P=\mathrm{L}(T M)$ ( $M$ manifold), resp. $P=\mathrm{O}(T M)$ ( $M$ Riemannian manifold), considered as manifolds, are parallelizable, i.e., the tangent bundles $T \mathrm{~L}(T M) \rightarrow \mathrm{L}(T M)$ and $T \mathrm{O}(T M) \rightarrow \mathrm{O}(T M)$ are trivial.

Proof. Indeed a $G$-connection in $P$ decomposes $T P=V \oplus H$. A canonical trivialization for $T P$ is given as follows: the vertical subbundle $V$ is trivialized by the standard-vertical vector fields $\hat{A}$ to $A$, where $A$ runs through a basis of $\mathfrak{g}$; the horizontal subbundle $H$ is trivialized by the standard-horizontal vector fields $L_{1}, \ldots, L_{n}$ in $\Gamma(T P)$ defined by

$$
L_{i}(u):=h_{u}\left(u e_{i}\right)
$$

For any $u \in P$, then

$$
\left(\hat{A}(u), L_{i}(u): A \in \text { basis for } \mathfrak{g}, i=1, \ldots, n\right)
$$

is a basis for $T_{u} P=V_{u} \oplus H_{u}$ which is obvious from the isomorphisms $\mathfrak{g} \stackrel{\sim}{\longrightarrow} V_{u}$, $A \mapsto \hat{A}(u)$ and $h_{u}: T_{\pi(u)} M \stackrel{\sim}{\longrightarrow} H_{u}$.

Remark 4.24. The standard-vertical, resp., standard-horizontal vector fields are determined by the relations

$$
\vartheta(\hat{A})=0 \text { and } \vartheta\left(L_{i}\right)=e_{i} \text { resp. } \omega(\hat{A})=A \text { and } \omega\left(L_{i}\right)=0 .
$$

The canonical second order partial differential operator $\Delta^{\text {hor }}:=\sum_{i} L_{i}^{2}$ is called horizontal Laplacian on $\mathrm{L}(T M)$, resp. $\mathrm{O}(T M)$.

Definition 4.25 (Horizontal lift of an $M$-valued semimartingale). For any $P$ valued semimartingale $U$ the Stratonovich integral $\int_{U} \omega$ (defined componentwise with respect to a basis of $\mathfrak{g}$ ) gives a semimartingale taking values in the Lie algebra $\mathfrak{g}$. We call $U$ horizontal if $\int_{U} \omega=0$ a.s. For an $M$-valued semimartingale $X$, a semimartingale $U$ taking values in $P$ is called horizontal lift of $X$, if $U$ is horizontal and if $\pi \circ U=X$ a.s.

Remark 4.26. Definition 4.25 generalizes the classical notion of horizontal lift for $M$-valued differentiable curves: a curve $t \mapsto u(t)$ over $t \mapsto x(t)$ is called horizontal if $\pi \circ u=x$ and $\omega(\dot{u})=0$.

For the remainder of this subsection we deal with the following situation: $M$ will either be a differentiable manifold equipped with a torsion-free connection, or $M$ will be a Riemannian manifold equipped with the Levi-Civita connection.

Definition 4.27 (Anti-development of an $M$-valued semimartingale). Let $X$ be an $M$-valued semimartingale and $U$ a horizontal lift of $X$ taking values in $P=\mathrm{L}(T M)$, resp. $\mathrm{O}(T M)$. The $\mathbb{R}^{n}$-valued semimartingale

$$
Z=\int_{U} \vartheta \equiv \int \vartheta(\circ d U)
$$

is called anti-development of $X$ into $\mathbb{R}^{n}$ (with respect to the initial frame $U_{0}$ ). In terms of the standard basis of $\mathbb{R}^{n}$ we have $Z \equiv\left(Z^{1}, \ldots, Z^{n}\right)$ where $Z^{i}=\int_{U} \vartheta^{i}$. 
Theorem 4.28. Let $X$ be an $M$-valued semimartingale, $U$ a horizontal lift of $X$ to $P=\mathrm{L}(T M)$ resp. $\mathrm{O}(T M)$, and $Z$ an anti-development of $X$ into $\mathbb{R}^{n}$. The following statements hold:

(i) $\int_{U} \sigma=\sum_{i=1}^{n} \int \sigma(U) L_{i}(U) \circ d Z^{i}$ for each differential form $\sigma \in \Gamma\left(T^{*} P\right)$.

(ii) $\int_{X} \alpha=\sum_{i=1}^{n} \int \alpha(X) U e_{i} \circ d Z^{i}$ for each differential form $\alpha \in \Gamma\left(T^{*} M\right)$.

In particular, $d(f \circ U)=\sum_{i=1}^{n}\left(L_{i} f\right)(U) \circ d Z^{i}$ for each function $f \in C^{\infty}(P)$, or in short-terms

$$
d U=\sum_{i=1}^{n} L_{i}(U) \circ d Z^{i},
$$

as well as $d(f \circ X)=\sum_{i=1}^{n}\left(U e_{i}\right)(f) \circ d Z^{i}$ for each function $f \in C^{\infty}(M)$, or in short-terms

$$
d X=U \circ d Z \text {. }
$$

Proof. The additional claims follow from (i) and (ii) with $\sigma=d f$ where $f \in C^{\infty}(P)$, resp. $\alpha=d f$ where $f \in C^{\infty}(M)$.

To (i): According to Theorem 4.7 it is sufficient that the right-hand side of (i) has the defining properties of $\int_{U} \sigma$. For $f \in C^{\infty}(P)$ we have to show that

$$
d(f \circ U)=\sum_{i}(d f)(U) L_{i}(U) \circ d Z^{i} \equiv \sum_{i}\left(L_{i} f\right)(U) \circ d Z^{i}
$$

which is equivalent to

$$
f \circ U-f \circ U_{0}=\int_{U} \sigma \quad \text { where } \sigma \in \Gamma\left(T^{*} P\right), \sigma_{u}:=\sum_{i}\left(L_{i} f\right)(u) \vartheta_{u}^{i} .
$$

But we observe that $\sum_{i}\left(L_{i} f\right)(u) \vartheta_{u}^{i}=(d f)_{u} \circ \operatorname{pr}_{H_{u}}$, indeed for $A \in T_{u} P$ we have

$$
\begin{aligned}
\sum_{i}\left(L_{i} f\right)(u) \vartheta_{u}^{i}(A) & =\sum_{i}(d f)_{u} L_{i}(u) \vartheta_{u}^{i}(A) \\
& =\sum_{i}(d f)_{u} h_{u}\left(u e_{i}\right)\left(u^{-1}(d \pi)_{u} A\right)^{i} \\
& =(d f)_{u} h_{u}\left(u u^{-1}(d \pi)_{u} A\right) \\
& =(d f)_{u} h_{u}\left((d \pi)_{u} A\right) \\
& =\left((d f)_{u} \circ \operatorname{pr}_{H_{u}}\right)(A) .
\end{aligned}
$$

On the other side, we have $\left(d f \circ \operatorname{pr}_{V}\right)_{u}=(d f)_{u} \kappa_{u} \omega_{u}=d\left(f \circ I_{u}\right)_{e} \omega_{u}$. But $U$ is horizontal and hence $\int_{U} d f \circ \operatorname{pr}_{V}=0$ which shows that

$$
f \circ U-f \circ U_{0}=\int_{U} d f=\int_{U} d f \circ \operatorname{pr}_{H}+\int_{U} d f \circ \operatorname{pr}_{V}=\int_{U} d f \circ \operatorname{pr}_{H}=\int_{U} \sigma .
$$

The second defining property of the Stratonovich integral is obvious.

To (ii): It is sufficient to show that

$$
d(f \circ X)=\sum_{i}(d f)(X) U e_{i} \circ d Z^{i} \equiv \sum_{i}\left(U e_{i}\right)(f) \circ d Z^{i}
$$


holds for each function $f \in C^{\infty}(M)$. With part (i) using that $(d \pi)_{u} L_{i}(u)=u e_{i}$, we obtain

$$
\begin{aligned}
d(f \circ \pi \circ U) & =\sum_{i} d(f \circ \pi)(U) L_{i}(U) \circ d Z^{i} \\
& =\sum_{i}(d f)(\pi(U))(d \pi)(U) L_{i}(U) \circ d Z^{i} \\
& =\sum_{i}(d f)(X) U e_{i} \circ d Z^{i},
\end{aligned}
$$

which shows the claim.

Theorem 4.29. Let $X$ be an $M$-valued semimartingale, $U$ a horizontal lift of $X$ to $P=\mathrm{L}(T M)$ resp. $\mathrm{O}(T M)$, and $Z$ an anti-development of $X$ into $\mathbb{R}^{n}$. Then

(i) $\int a(d U, d U)=\sum_{i, j=1}^{n} \int a(U)\left(L_{i}(U), L_{j}(U)\right) d\left[Z^{i}, Z^{j}\right]$ for $a \in \Gamma\left(T^{*} P \otimes T^{*} P\right)$.

(ii) $\int b(d X, d X)=\sum_{i, j=1}^{n} \int b(X)\left(U e_{i}, U e_{j}\right) d\left[Z^{i}, Z^{j}\right]$ for $b \in \Gamma\left(T^{*} M \otimes T^{*} M\right)$.

Proof. It is again sufficient to consider the special case $a=d \varphi_{1} \otimes d \varphi_{2}$ where $\varphi_{1}, \varphi_{2} \in$ $C^{\infty}(P)$, resp. $b=d f_{1} \otimes d f_{2}$ where $f_{1}, f_{2} \in C^{\infty}(M)$. Then the statements follow with Remark 4.11.

Theorem 4.30 (Existence of horizontal lifts to $M$-valued semimartingales). Let $P$ be a $G$-principal bundle over a manifold $M$ endowed with a $G$-connection. Let $x_{0}$ be an $M$-valued random variable and $u_{0}$ a $P$-valued random variable over $x_{0}$, i.e. $\pi \circ u_{0}=x_{0}$ a.s. Then to each $M$-valued semimartingale $X$ with $X_{0}=x_{0}$ there is exactly one horizontal lift $U$ to $P$ with $U_{0}=u_{0}$ a.s.

Proof. See [53] or [24], Chapter 7. The existence part is straightforward. According to Theorem 2.22, the semimartingale $X$ can be realized as solution of a Stratonovich SDE of the form

$$
d X=\sum_{i=1}^{\ell} A_{i}(X) \circ d Z^{i}, \quad X_{0}=x_{0},
$$

where $Z$ is an $\mathbb{R}^{\ell}$-valued semimartingale for some $\ell$. Let $\bar{A}_{i} \in \Gamma(T P)$ be the horizontal lift of $A_{i} \in \Gamma(T M)$, i.e. $\bar{A}_{i}(u)=h_{u}\left(A_{i}(\pi u)\right)$ for $u \in P$, and consider the "horizontally lifted SDE" on $P$ :

$$
d U=\sum_{i=1}^{\ell} \bar{A}_{i}(U) \circ d Z^{i}, \quad U_{0}=u_{0} .
$$

It is clear that solutions to (4.24) are canonical candidates for the wanted horizontal lift. Indeed, we have $d(\pi \circ U)=\sum_{i}(d \pi)_{U} \bar{A}_{i}(U) \circ d Z^{i} \equiv \sum_{i} A_{i}(\pi \circ U) \circ d Z^{i}$ with $\pi \circ U_{0}=x_{0}$, and hence $\pi \circ U=X$ by uniqueness of solutions to (4.23). On the other hand, we have $\int_{U} \omega=\sum_{i} \int \omega(U) \bar{A}_{i}(U) \circ d Z^{i}=0$. It remains to verify that $U$ and $X$ have identical lifetimes.

We want to summarize the theory developed so far. Let $M$ be a differentiable manifold equipped with a torsion-free connection, or a Riemannian manifold with the Levi-Civita connection. To a semimartingale $X$ on $M$ we defined its horizontal lift $U$ to $P=\mathrm{L}(T M)$, resp. $\mathrm{O}(T M)$, and its anti-development $Z$ into $\mathbb{R}^{n}$. Then (modulo choice of initial conditions $X_{0}=x, U_{0}=u$ ) each of the three processes $X, U, Z$ determines the two others. 
Indeed, we have:

(a) $Z$ determines $U$ as solution to the $\operatorname{SDE} d U=\sum_{i=1}^{n} L_{i}(U) \circ d Z^{i}$ with $U_{0}=u$,

(b) $U$ determines $X$ via $X=\pi \circ U$,

(c) $X$ determines $Z$ as $Z=\int_{U} \vartheta$ where $U$ is the unique horizontal lift of $X$ to $P$ with $U_{0}=u$.

Typically, one starts with $Z$ on $\mathbb{R}^{n}$ to determine $X$ on $M$ (stochastic development of $Z$ ). The frame $U$ moves along $X$ by parallel transport.

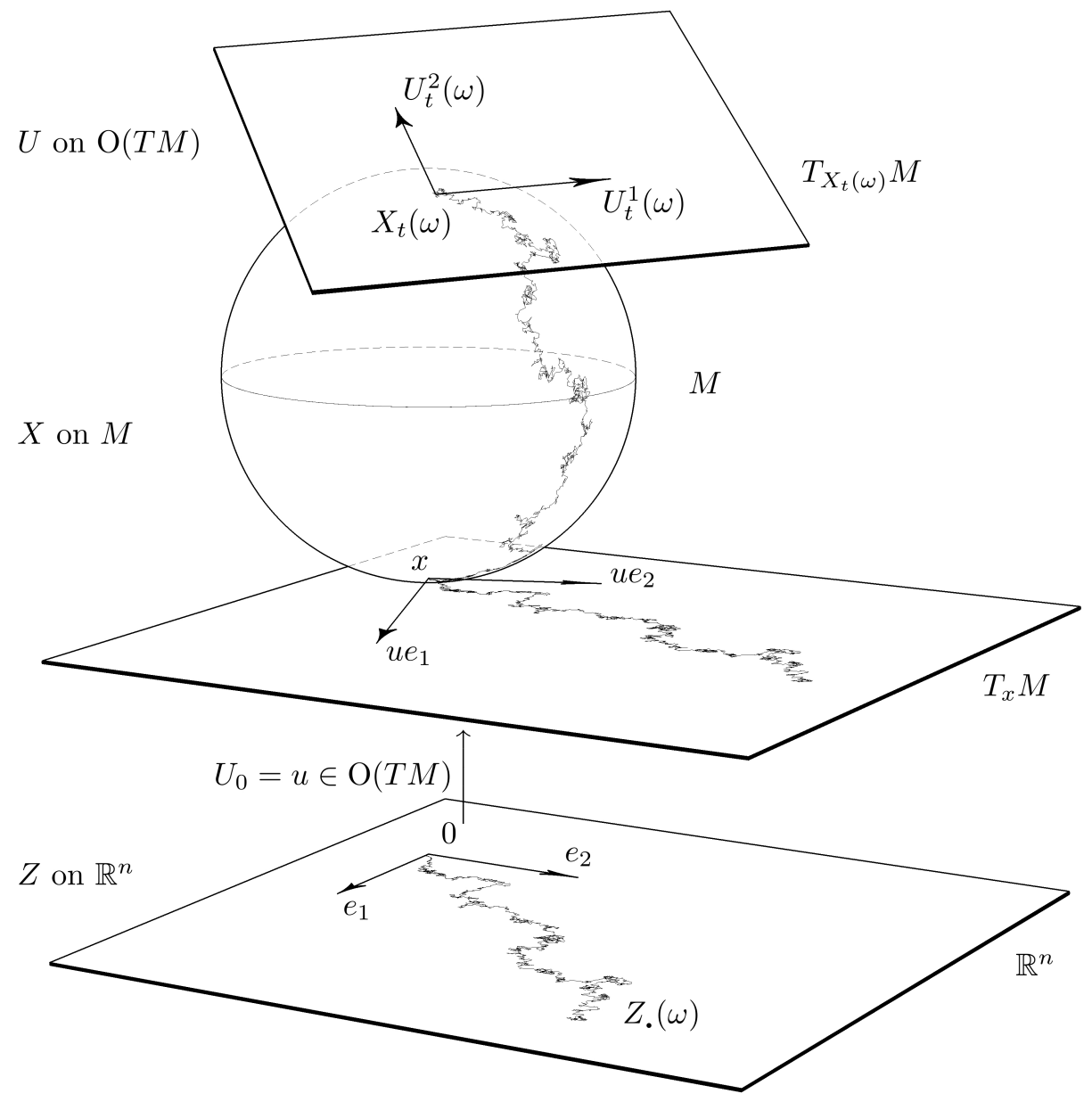

In the deterministic special case of a differentiable curve $Z: t \mapsto z(t)$ in $\mathbb{R}^{n}$ stochastic development reduces to the canonical Cartan development of $z(t)$.

Example 4.31 (Cartan development). The Cartan development of an $\mathbb{R}^{n}$-valued curve $t \mapsto z(t)$ is the construction of curves $x: t \mapsto x(t) \in M$ and $u: t \mapsto u(t) \in P$ (where $P=\mathrm{L}(T M)$, resp. $P=\mathrm{O}(T M)$ in the Riemannian case) such that $u(\cdot)$ lies above $x(\cdot)$ and such that

(i) $\dot{x}=u \dot{z}$, or in equivalent notation $d x(t)=u(t) d z(t)$,

(ii) $u$ is parallel along $x$, i.e., $\nabla_{D} u \equiv\left(\nabla_{D} u^{1}, \ldots, \nabla_{D} u^{n}\right)=0$ where $D=\partial / \partial t$.

Condition (ii) means that $u(\cdot)$ is a horizontal curve; thus $\dot{u} \in H_{u} \equiv h_{u}\left(T_{\pi(u)} M\right)$, and hence $\dot{u}=h_{u}(\dot{x})=h_{u}(u \dot{z})$ by using (i). Since $h_{u}(u \dot{z})=\sum_{i} h_{u}\left(u e_{i}\right) \dot{z}^{i}=$ 
$\sum_{i} L_{i}(u) \dot{z}^{i}$, conditions (i) and (ii) are seen to be equivalent to

$$
d u=\sum_{i} L_{i}(u) d z^{i}
$$

Definition 4.32 (Parallel transport along a semimartingale). Let $M$ be a differentiable manifold equipped with a torsion-free connection, or a Riemannian manifold with the Levi-Civita connection. Let $X$ be a semimartingale on $M$ and $U$ an arbitrary horizontal lift of $X$ to $\mathrm{L}(T M)$ resp. $\mathrm{O}(T M)$. For $0 \leq s \leq t$ let $/ / s, t:=U_{t} \circ U_{s}^{-1}$ given by

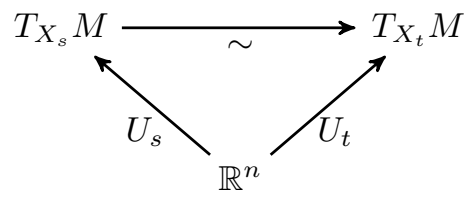

The isomorphisms (resp. isometries in the Riemannian case)

$$
/ /_{0, t}: T_{X_{0}} M \rightarrow T_{X_{t}} M
$$

are called stochastic parallel transport along $X$.

Theorem 4.33 (Geometric Itô formula). Let $M$ be a differentiable manifold equipped with a linear connection $\nabla$ (without restriction $\nabla$ torsion-free). Let $X$ be an $M$ valued semimartingale, $U$ a horizontal lift of $X$ to $\mathrm{L}(T M)$ and $Z=\int_{U} \vartheta$ the corresponding anti-development of $X$ into $\mathbb{R}^{n}$. For each $f \in C^{\infty}(M)$ the following formula hold:

$$
d(f \circ X)=\sum_{i=1}^{n}(d f)(X)\left(U e_{i}\right) d Z^{i}+\frac{1}{2} \sum_{i, j=1}^{n}(\nabla d f)(X)\left(U e_{i}, U e_{j}\right) d\left[Z^{i}, Z^{j}\right],
$$

or in abbreviated form (see Theorem 4.29),

$$
d(f \circ X)=(d f)(U d Z)+\frac{1}{2} \nabla d f(d X, d X) .
$$

Proof. From $d U=\sum_{i} L_{i}(U) \circ d Z^{i}$ we first see that

$$
\begin{aligned}
& d(f \circ X)=d(f \circ \pi \circ U)=\sum_{i} L_{i}(f \circ \pi)(U) \circ d Z^{i} \\
& \quad=\sum_{i} L_{i}(f \circ \pi)(U) d Z^{i}+\frac{1}{2} \sum_{i, j} L_{i} L_{j}(f \circ \pi)(U) d\left[Z^{i}, Z^{j}\right]
\end{aligned}
$$

where $L_{i}(f \circ \pi)(u)=d(f \circ \pi)_{u} L_{i}(u)=(d f)_{\pi(u)}(d \pi)_{u} h_{u}\left(u e_{i}\right)=(d f)_{\pi(u)}\left(u e_{i}\right)$. A straightforward calculation however shows that

$$
L_{i} L_{j}(f \circ \pi)(u)=\nabla d f\left(u e_{i}, u e_{j}\right),
$$

from where formula (4.25) results.

Remark 4.34. Let $M$ be a Riemannian manifold with its Levi-Civita connection. Denoting by $\Delta^{\text {hor }}=\sum_{i} L_{i}^{2}$ the horizontal Laplacian on $\mathrm{O}(T M)$ and by $\Delta$ the Laplace-Beltrami operator on $M$, then for each $f \in C^{\infty}(M)$ the following relation holds:

$$
\Delta^{\text {hor }}(f \circ \pi)=(\Delta f) \circ \pi .
$$

Proof. Indeed, for $u \in \mathrm{O}(T M)$, we have

$$
\sum_{i} L_{i}^{2}(f \circ \pi)(u)=\sum_{i} \nabla d f\left(u e_{i}, u e_{i}\right)=(\operatorname{trace} \nabla d f) \pi(u)=(\Delta f) \circ \pi(u) .
$$


Theorem 4.35. Let $M$ be a differentiable manifold equipped with a torsion-free linear connection $\nabla$. Let $X$ be an $M$-valued semimartingale and $U_{0}$ an $\mathrm{L}(T M)$ valued $\mathscr{F}_{0}$-measurable random variable such that $\pi \circ U_{0}=X_{0}$ a.s.; furthermore let $Z=\int_{U} \vartheta$ be the anti-development of $X$ into $\mathbb{R}^{n}$ with respect to the initial frame $U_{0}$. Then

(i) $X$ is a $\nabla$-martingale on $M$ if and only if $Z$ is a local martingale on $\mathbb{R}^{n}$.

(ii) If $\nabla$ is the Levi-Civita connection to some Riemannian metric $g$ on $M$ and if $U_{0}$ takes its values in $\mathrm{O}(T M)$, then $X$ is a Brownian motion on $(M, g)$ if and only if $Z$ is a Brownian motion on $\mathbb{R}^{n}$ (more precisely, a Brownian motion on $\mathbb{R}^{n}$ stopped at the lifetime $\zeta$ of $\left.X\right)$.

Proof. (i) According to Definition $4.13 X$ is a $\nabla$-martingale, if

$$
d(f \circ X)-\frac{1}{2}(\nabla d f)(d X, d X) \stackrel{m}{=} 0 .
$$

for functions $f \in C^{\infty}(M)$. By means of the geometric Itô's formula 4.33 this means that

$$
\sum_{i}(d f)(X)\left(U e_{i}\right) d Z^{i} \stackrel{\mathrm{m}}{=} 0
$$

for any $f \in C^{\infty}(M)$ which is easily seen to be equivalent to the condition that $Z$ is a local martingale.

(ii) According to Theorem $4.17 X$ is a Brownian motion on $(M, g)$ if

$$
d(f \circ X)-\frac{1}{2}(\Delta f \circ X) d t \stackrel{\mathrm{m}}{=} 0
$$

for all $f \in C^{\infty}(M)$. According to formula (4.25), clearly if $Z$ is a Brownian motion $\mathbb{R}^{n}$, then $X$ will be Brownian motion on $(M, g)$. Conversely, if $X$ is Brownian motion on $(M, g)$ then by Lévy's characterization of $M$-valued Brownian motions (Theorem 4.17) $X$ is a $\nabla$-martingale, and thus $Z$ a local martingale by part (i). On the other hand, we have $Z^{i}=\int_{U} \vartheta^{i}$ where $\vartheta_{u}^{i}=\left\langle d \pi(\cdot), u e_{i}\right\rangle=\pi^{*}\left\langle\cdot, u e_{i}\right\rangle$. We may calculate the quadratic variation of $Z$ using Remark 4.11 as follows:

$$
\begin{aligned}
d\left[Z^{i}, Z^{j}\right] & =d\left[\int_{U} \vartheta^{i}, \int_{U} \vartheta^{j}\right]=\left(\vartheta^{i} \otimes \vartheta^{j}\right)(d U, d U) \\
& =\pi^{*}\left(\left\langle\cdot, U e_{i}\right\rangle \otimes\left\langle\cdot, U e_{j}\right\rangle\right)(d U, d U) \\
& =\left(\left\langle\cdot, U e_{i}\right\rangle \otimes\left\langle\cdot, U e_{j}\right\rangle\right)(d X, d X) \\
& =\operatorname{trace}\left(\left\langle\cdot, U e_{i}\right\rangle \otimes\left\langle\cdot, U e_{j}\right\rangle\right)(X) d t=\delta_{i j} d t .
\end{aligned}
$$

By means of Lévy's characterization for Brownian motions on $\mathbb{R}^{n}$ we see that $Z$ Brownian motion.

Theorem 4.35 provides a canonical construction of Brownian motions on Riemannian manifolds. One obtains Brownian motions on $(M, g)$ with starting point $x \in M$ as stochastic development of a Brownian motion $B$ on $\mathbb{R}^{n}$ as follows. Choose $u \in \mathrm{O}(T M)$ such that $\pi(u)=x$ and solve the SDE

$$
d U=\sum_{i=1}^{n} L_{i}(U) \circ d B^{i}, \quad U_{0}=u .
$$

According to Theorem 4.35 then $X=\pi \circ U$ will be a Brownian motion on $(M, g)$ starting from $X_{0}=x$.

Remark 4.36. Let $X$ be an $M$-valued semimartingale with starting point $x \in M$. The anti-development $Z$ of $X$ into $\mathbb{R}^{n}$ (see Definition 4.27) required the choice of a frame $u$ above $x$,

$$
Z=\int_{U} \vartheta, \quad U_{0}=u
$$


Considering the anti-development of $X$ into $T_{x} M$, i.e.

$$
Z^{\prime}=U_{0} \int_{U} \vartheta
$$

makes the notion intrinsic. Then we have the formula

$$
d Z^{\prime}=U_{0} U_{t}^{-1} \circ d X=/ /_{0, t}^{-1} \circ d X .
$$

4.4. Subelliptic diffusions and sub-Riemannian Brownian motions. In this subsection we want to adapt the results developed to far from the Riemannian to the sub-Riemannian setting.

A sub-Riemannian structure on a differentiable manifold $M$ is a pair $(\mathcal{H}, g)$ where $\mathcal{H}$ is a subbundle of $T M$ and $g$ a positive definite metric tensor defined only on $\mathcal{H}$. Any sub-Riemannian structure induces a vector bundle morphism

$$
\sharp: T^{*} M \rightarrow T M,
$$

determined by the properties $\sharp\left(T^{*} M\right)=\mathcal{H}$ and $q(v)=g(v, \sharp q)$ for any $q \in T^{*} M$ and $v \in \mathcal{H}$. The kernel of $\sharp$ is the subbundle $\operatorname{Ann}(\mathcal{H}) \subseteq T^{*} M$ of elements of $T^{*} M$ vanishing on $\mathcal{H}$. Then the so-called co-metric $g^{*}$ on $T^{*} M$, defined by

$$
g^{*}\left(q_{1}, q_{2}\right)=q_{1}\left(\sharp q_{2}\right), \quad q_{1}, q_{2} \in T_{x}^{*} M, x \in M,
$$

degenerates along $\operatorname{Ann}(\mathcal{H})$. It is obvious that sub-Riemannian structures on $M$ and co-metrics degenerating along a subbundle of $T^{*} M$ are equivalent structures.

Definition 4.37. Let $(\mathcal{H}, g)$ be a sub-Riemannian structure on $M$. A continuous semimartingale $X$ taking values in $M$ is called horizontal, or a sub-Riemannian diffusion, if

$$
\int \alpha(\circ d X)=0 \quad \text { for all } \alpha \in \Gamma(\operatorname{Ann}(\mathcal{H})) .
$$

Here $\int \alpha(\circ d X) \equiv \int_{X} \alpha$ denotes the Stratonovich integral of $\alpha$ along $X$.

Remark 4.38. Note that if $X$ is a horizontal semimartingale then $\int_{X} \beta$ is welldefined for $\beta \in \Gamma\left(\mathcal{H}^{*}\right)$. The same holds true for $\int b(d X, d X)$ if $b \in \Gamma\left(\mathcal{H}^{*} \otimes \mathcal{H}^{*}\right)$ is a bilinear form on $\mathcal{H}^{*}$. In particular, the sub-Riemannian quadratic variation of $X$,

$$
[X, X]=\int g(d X, d X),
$$

is well-defined.

As seen in Theorem 2.22, a continuous semimartingale $X$ taking values in $M$ can always be obtained as solution of an SDE of the type $d X=\sum_{i} A_{i}(X) \circ d Z^{i}$. Then obviously $X$ is horizontal if the vector fields $A_{i}$ are horizontal in the sense that $A_{i} \in \Gamma(\mathcal{H})$.

To define horizontal martingales in the sub-Riemannian setting we need to specify a connection $\nabla$. To this end it is enough to have a so-called partial connection on $\mathcal{H}$ (see [32] and [20], Section 2)

$$
\Gamma(\mathcal{H}) \times \Gamma(\mathcal{H}) \rightarrow \Gamma(\mathcal{H}), \quad(A, B) \mapsto \nabla_{A} B,
$$

and correspondingly the partial Hessian of a function $f \in C^{\infty}(M)$,

$$
\nabla d f \equiv \operatorname{Hess} f \in \Gamma\left(\mathcal{H}^{*} \otimes \mathcal{H}^{*}\right), \quad(\nabla d f)(A, B)=A B f-\left(\nabla_{A} B\right) f, \quad f \in C^{\infty}(M) .
$$

Definition 4.39. Let $(\mathcal{H}, g)$ be a sub-Riemannian structure on $M$ and $\nabla$ a partial connection on $\mathcal{H}$. A continuous semimartingale $X$ taking values in $M$ is called a horizontal martingale if $H$ is horizontal and for any $f \in C^{\infty}(M)$,

$$
d(f \circ X)-\frac{1}{2}(\nabla d f)(d X, d X) \stackrel{\mathrm{m}}{=} 0 .
$$


Remark 4.40. (a) Often partial connections are induced from (full) connections $\tilde{\nabla}$ on $M$ in terms of a projection $p: T M \rightarrow \mathcal{H}$ as

$$
\nabla_{A} B=p\left(\tilde{\nabla}_{A} B\right), \quad A, B \in \Gamma(\mathcal{H}) .
$$

For instance, one may extend the metric $g$ from $\mathcal{H}$ to a full Riemannian metric $\tilde{g}$ on $T M$ (this is a common procedure in the case of sub-Riemannian structures related to Riemannian foliations) then

$$
\nabla_{A} B=\operatorname{pr}_{\mathcal{H}}\left(\tilde{\nabla}_{A} B\right), \quad A, B \in \Gamma(\mathcal{H}),
$$

(where $\tilde{\nabla}$ is the Levi-Civita connection to $\tilde{g}$ on $M$ and $\operatorname{pr}_{\mathcal{H}}$ the orthogonal projection of $T M$ onto $\mathcal{H}$ ) defines a partial connection on $\mathcal{H}$ which is moreover metric, i.e. $\nabla_{A} g=0$ for all $A \in \Gamma(\mathcal{H})$. Note that $(4.28)$ is the horizontal part of the so-called Bott connection on $M$, see [57], Chapt. 5 .

(b) More generally, it is straight-forward to show the following result. Given a projection $p: T M \rightarrow \mathcal{H}$, there exists a unique partial connection on $\mathcal{H}$ which is metric and has the property

$$
\nabla_{A} B-\nabla_{B} A-p[A, B]=0 .
$$

This is actually the connection in (4.28) defined relative to any Riemannian metric $\tilde{g}$ such that $p$ is the orthogonal projection.

Theorem 4.17 is now easily adapted to the sub-Riemannian setting. Given a partial connection $\nabla$ on $\mathcal{H}$ which is metric (i.e. $\nabla_{A} g=0$ for all $A \in \Gamma(\mathcal{H})$ ) we define the sub-Laplacian $\Delta_{\mathcal{H}}$ relative to $(\mathcal{H}, g, \nabla)$ as

$$
\Delta_{\mathcal{H}} f=\operatorname{trace}_{\mathcal{H}} \nabla d f, \quad f \in C^{\infty}(M) .
$$

If the partial connection $\nabla$ is as in Remark 4.40 (b), then $\Delta_{\mathcal{H}}$ coincides with the sub-Laplacian relative to the complement $\mathcal{V}=\operatorname{ker} p$ as defined in [22], Section 2.2.

Theorem 4.41 (Lévy's characterization of sub-Riemannian Brownian motions on $M)$. Let $(\mathcal{H}, g)$ be a sub-Riemannian structure on $M$ and $\nabla$ a partial metric connection on $\mathcal{H}$. For a horizontal semimartingale $X$ of maximal lifetime on $M$ the following conditions are equivalent:

(i) $X$ is a sub-Riemannian Brownian motion on $M$, i.e. for any $f \in C^{\infty}(M)$, the real-valued process

$$
f \circ X-\frac{1}{2} \int\left(\Delta_{\mathcal{H}} f\right) \circ X d t
$$

is a local martingale.

(ii) $X$ is a $\nabla$-martingale such that $[f(X), f(X)]=\int g^{*}(d f, d f)\left(X_{t}\right) d t$ for every $f \in C^{\infty}(M)$.

(iii) $X$ is a $\nabla$-martingale such that $\int b(d X, d X)=\int\left(\operatorname{trace}_{\mathcal{H}} b\right)(X) d t$ for every $b \in \Gamma\left(\mathcal{H}^{*} \otimes \mathcal{H}^{*}\right)$.

In particular, for the sub-Riemannian quadratic variation (4.27) of $X$, we then have

$$
\int_{0}^{t} g(d X, d X)=t \operatorname{dim} \mathcal{H}
$$

Analogously to Theorem 4.18, we can construct sub-Riemannian Brownian motion on $M$ as solutions to SDEs.

Theorem 4.42 (Sub-Riemannian Brownian motions as solutions of an SDE on $M$ ). Let $(\mathcal{H}, g)$ be a sub-Riemannian structure on $M$ and $\nabla$ a partial metric connection on $\mathcal{H}$. Consider an SDE of the type

$$
d X=A_{0}(X) d t+A(X) \circ d B
$$


with $A_{0} \in \Gamma(\mathcal{H})$ and $A \in \Gamma\left(\operatorname{Hom}\left(M \times \mathbb{R}^{r}, \mathcal{H}\right)\right)$; the driving process $B$ is a Brownian motion on $\mathbb{R}^{r}$ (for some $r$ ).

Then maximal solutions to (4.29) are sub-Riemannian Brownian motion $M$ if the two following conditions are satisfied:

(i) $A_{0}=-\frac{1}{2} \sum_{i} \nabla_{A_{i}} A_{i}$ with $A_{i} \equiv A(\cdot) e_{i}$ for $i=1, \ldots, r$.

(ii) The map $A(x)^{*}: \mathcal{H}_{x} \rightarrow \mathbb{R}^{r}$ is an isometric embedding for every $x \in M$, i.e. $A(x) A(x)^{*}=\mathrm{id}_{\mathcal{H}_{x}}$ where $A(x)^{*}$ is the adjoint to $A(x) \in \operatorname{Hom}\left(\mathbb{R}^{r}, \mathcal{H}_{x}\right)$.

The problem of defining sub-Riemannian Brownian motions and corresponding random walk approximations has recently been addressed in [15].

The results of Subsection 4.3 easily carry over to the case of horizontal martingales and sub-Riemannian Brownian motions. Instead of $\mathrm{L}(T M)$, resp. $\mathrm{O}(T M)$, we work with the $G$-principal bundle $P=\mathrm{L}(\mathcal{H})$ of frames in $\mathcal{H}$, resp. $P=\mathrm{O}(\mathcal{H})$ of orthonormal frames in $\mathcal{H}$, where now $G=\mathrm{GL}(k ; \mathbb{R})$, resp. $G=\mathrm{O}(k ; \mathbb{R})$, and $k=\operatorname{dim} \mathcal{H}$. In other words,

$$
P_{x}=\left\{u: \mathbb{R}^{k} \rightarrow \mathcal{H}_{x} \mid u \text { linear isomorphisms, resp. } u \text { linear isometry }\right\}, \quad x \in M .
$$

A partial connection $\nabla$ on $\mathcal{H}$, resp. a metric partial connection $\nabla$ on $\mathcal{H}$, induces now a $G$-invariant subbundle $H \subset T P$ such that

$$
\pi_{*}: H_{u} \stackrel{\sim}{\longrightarrow} \mathcal{H}_{\pi(u)}
$$

where $\pi$ is the projection $P \rightarrow M$. In terms of the horizontal lift of this $G$ connection,

$$
h: \pi^{*} \mathcal{H} \stackrel{\sim}{\longrightarrow} \hookrightarrow T P
$$

we have the standard-horizontal vector fields

$$
L_{i} \in \Gamma(T P), \quad L_{i}(u)=h_{u}\left(u e_{i}\right), u \in P, i=1, \ldots, k .
$$

The $\mathfrak{g}$-valued connection form $\omega$ and the $\mathbb{R}^{k}$-valued canonical 1-form $\vartheta$ are defined as in the Riemannian case, but for a partial connection they are given only on $H \oplus V$ with $V=\operatorname{ker} d \pi$, and no longer globally on $T P$, in other words

$$
\omega \in \Gamma\left(\left(H^{*} \oplus V^{*}\right) \otimes \mathfrak{g}\right) \quad \text { and } \quad \vartheta \in \Gamma\left(\left(H^{*} \oplus V^{*}\right) \otimes \mathbb{R}^{k}\right) .
$$

One can now define stochastic developments of $\mathbb{R}^{k}$-valued semimartingales according to

$$
\begin{aligned}
& d U=\sum_{i=1}^{k} L_{i}(U) \circ d Z^{i}, \quad U_{0}=u, \\
& X=\pi(U),
\end{aligned}
$$

as we did in Subsection 4.3. The resulting processes $X$ will be horizontal semimartingales on $M$. Horizontal lifts of such semimartingales $X$ to $P=\mathrm{L}(\mathcal{H})$ can be established as in the Riemannian case, for instance, by representing $X$ as solution to an SDE on $M$ with vector fields $A_{i} \in \Gamma(\mathcal{H})$ and solving the "horizontally lifted" SDE on $P$ (see proof to Theorem 4.30).

Theorem 4.43 (Geometric Itô formula for horizontal diffusions). Let $(\mathcal{H}, g)$ be a sub-Riemannian structure on $M$ and $\nabla$ a partial connection on $\mathcal{H}$. Let $X$ be an $M$-valued horizontal semimartingale, $U$ a horizontal lift of $X$ to $P=\mathrm{L}(\mathcal{H})$ and $Z=\int_{U} \vartheta$ the corresponding anti-development of $X$ into $\mathbb{R}^{k}$. For each $f \in C^{\infty}(M)$ the following formula hold:

$$
d(f \circ X)=\sum_{i=1}^{k}(d f)(X)\left(U e_{i}\right) d Z^{i}+\frac{1}{2} \sum_{i, j=1}^{k}(\nabla d f)(X)\left(U e_{i}, U e_{j}\right) d\left[Z^{i}, Z^{j}\right],
$$


or in abbreviated form,

$$
d(f \circ X)=(d f)(U d Z)+\frac{1}{2} \nabla d f(d X, d X) .
$$

This finally gives the following sub-Riemannian version of Theorem 4.35.

Theorem 4.44. Let $(\mathcal{H}, g)$ be a sub-Riemannian structure on $M$ and $\nabla$ a partial connection on $\mathcal{H}$. Let $X$ be an $M$-valued horizontal semimartingale and $U_{0}$ an $\mathrm{L}(\mathcal{H})$-valued $\mathscr{F}_{0}$-measurable random variable such that $\pi \circ U_{0}=X_{0}$ a.s.; furthermore let $Z=\int_{U} \vartheta$ be the anti-development of $X$ into $\mathbb{R}^{k}$ with respect to the initial frame $U_{0}$. Then

(i) $X$ is a $\nabla$-martingale on $M$ if and only if $Z$ is a local martingale on $\mathbb{R}^{k}$.

(ii) If $\nabla$ is a metric partial connection on $\mathcal{H}$ and if $U_{0}$ takes its values in $\mathrm{O}(\mathcal{H})$, then $X$ is a sub-Riemannian Brownian motion on $M$ if and only if $Z$ is a Brownian motion on $\mathbb{R}^{k}$ (more precisely, a Brownian motion on $\mathbb{R}^{k}$ stopped at the lifetime $\zeta$ of $X)$.

Following Remark 4.36 we have the following remark.

Remark 4.45. Let $\nabla$ be a partial connection on $\mathcal{H}$ and let be $X$ an $M$-valued horizontal semimartingale with starting point $x \in M$. Let $Z$ be the anti-development of $X$ into $\mathcal{H}_{x}$,

$$
Z=\int / /_{0, t}^{-1} \circ d X
$$

(a) Then $X$ is a $\nabla$-martingale on $M$ if and only if its anti-development $Z$ into $\mathcal{H}_{x}$ is a local martingale in $\mathcal{H}_{x}$.

(b) If $\nabla$ is a metric partial connection on $\mathcal{H}$, then $X$ is a sub-Riemannian Brownian motion on $M$ if and only if its anti-development $Z$ into $\mathcal{H}_{x}$ is a Brownian motion in $\mathcal{H}_{x}$.

Here $/ /_{0, t}: \mathcal{H}_{X_{0}} \stackrel{\sim}{\longrightarrow} \mathcal{H}_{X_{t}}$ denotes the stochastic parallel transport of horizontal tangent vectors along $X$. Recall that the $/ /_{0, t}$ are linear isomorphisms for a partial connection, and isometries for a metric partial connection.

\section{Control theory and support theorems}

5.1. Control systems. Consider a Stratonovich SDE on $M$ of the type

$$
d X=A_{0}(X) d t+\sum_{i=1}^{r} A_{i}(X) \circ d B^{i}
$$

driven by a Brownian motion $B=\left(B^{1}, \ldots, B^{r}\right)$ on $\mathbb{R}^{r}$.

Definition 5.1. Solutions $X$ to SDE (5.1) are called hypoelliptic diffusions if the vector fields $A_{1}, \ldots, A_{r}$ are bracket-generating in the sense that

$$
\operatorname{dim} \operatorname{Lie}\left(A_{1}, \ldots, A_{r}\right)(x)=\operatorname{dim} M \text { for all } x \in M \text {. }
$$

To the SDE (5.1) we associate the following control system

$$
\dot{x}(t)=A_{0}(x(t))+\sum_{i=1}^{r} A_{i}(x(t)) u^{i}(t)
$$

where the control $u=u(\cdot)$ lies in

$$
\mathcal{U}=\left\{u: \mathbb{R}_{+} \rightarrow \mathbb{R}^{r} \text { piecewise constant }\right\},
$$

see for instance [56]. In the space $\mathcal{U}$ of controls we could have equally taken $u$ piecewise smooth or piecewise continuous with values in $\mathbb{R}^{r}$.

We denote by 
- $X_{t}(x)$ the solution to SDE (5.1) with starting point $X_{0}=x$, and by

- $\phi_{t}(x, u)$ the solution to the control system (5.3) with initial condition $x(0)=x$ and $u=u(\cdot) \in \mathcal{U}$.

For simplicity, all vector fields of the form

$$
A_{0}+\sum_{i=1}^{r} A_{i} u^{i}, \quad u \in \mathbb{R}^{r} \text { fixed ("frozen vector fields") }
$$

are assumed to be complete in the remainder of Section 5 .

We consider the following orbits:

$$
\begin{aligned}
& O^{+}(x):=\left\{y \in M: y=\phi_{t}(x, u), t \geq 0, u=u(\cdot) \in \mathcal{U}\right\} \quad \text { "forward orbit", } \\
& O_{t}^{+}(x):=\left\{y \in M: y=\phi_{t}(x, u), u=u(\cdot) \in \mathcal{U}\right\} \quad \text { "forward orbit at time } t .
\end{aligned}
$$

We call the control system (5.3)

- completely controllable if $O^{+}(x)=M$ for each $x \in M$,

- strongly controllable if $O_{t}^{+}(x)=M$ for each $t>0$ and each $x \in M$,

- completely accessible if $O^{+}(x)$ has non-void interior for each $x \in M$,

- strongly accessible if $O_{t}^{+}(x)$ has non-void interior for each $t>0$ and each $x \in M$.

Remark 5.2. Geometric Control Theory characterizes properties of control problems in terms of Lie-algebra conditions on the vector fields $A_{0}, \ldots, A_{r}$. For example, for system (5.3)

(1) complete accessibility holds if $\operatorname{dim} \operatorname{Lie}\left(A_{0}, A_{1}, \ldots, A_{r}\right)(x)=\operatorname{dim} M$ for each $x \in M$,

(2) strong accessibility holds if $\operatorname{dim} \operatorname{Lie}\left(A_{0}+\frac{\partial}{\partial t}, A_{1}, \ldots, A_{r}\right)(t, x)=\operatorname{dim} M+1$ for each $t>0$ and $x \in M$,

(3) strong controllability holds if $\operatorname{dim} \operatorname{Lie}\left(A_{1}, \ldots, A_{r}\right)=\operatorname{dim} M$ for each $x \in M$.

See for instance $[2,16,31]$.

5.2. Support theorems. The famous support theorem of Stroock-Varadhan (1972) establishes a bridge between the theory of SDEs and control theory, more precisely, between Eq. (5.1) and Eq. (5.3).

Induced by $X .(x): \Omega \longrightarrow C\left(\mathbb{R}_{+}, M\right)$, we have the following measures:

$$
\begin{aligned}
\mathbb{P}_{x} & :=\mathbb{P} \circ X_{.}(x)^{-1} \text { probability measure on } C_{x}\left(\mathbb{R}_{+}, M\right), \\
\mathbb{P}_{t, x} & :=\mathbb{P} \circ X_{t}(x)^{-1} \text { probability measure on } M .
\end{aligned}
$$

Here $C_{x}\left(\mathbb{R}_{+}, M\right)$ denotes the space of continuous trajectories $\mathbb{R}_{+} \rightarrow M$ starting from $x$ at time zero.

Theorem 5.3 (Support theorem; Stroock-Varadhan [55]). For the supports of the probability measures $\mathbb{P}_{x}$, resp. $\mathbb{P}_{t, x}$, the following properties hold:

I. (Path space) On $C_{x}\left(\mathbb{R}_{+}, M\right)$ we have

$$
\operatorname{supp} \mathbb{P}_{x}=\overline{\{\phi .(x, u): u \in \mathcal{U}\}}
$$

II. (State space) On $M$ we have

$$
\begin{aligned}
\operatorname{supp} \mathbb{P}_{t, x} & =\bar{O}_{t}^{+}(x) \\
\operatorname{supp} G_{\lambda}(x, \cdot) & =\bar{O}^{+}(x)
\end{aligned}
$$

where

$$
G_{\lambda}(x, \cdot)=\int_{0}^{\infty} e^{-\lambda t} \mathbb{P}_{t, x}(\cdot) d t, \quad \lambda>0,
$$

denotes the Green's measure with exponent $\lambda$ on $M$. 
Proof. The support theorem is proved by approximating the driving Brownian motion $B$ through its piecewise linear polygonal approximation

$$
B_{t}^{\pi}=\left(t_{i+1}-t_{i}\right)^{-1}\left[\left(t_{i+1}-t\right) B_{t_{i}}+\left(t-t_{i}\right) B_{t_{i+1}}\right], \quad t_{i} \leq t \leq t_{i+1},
$$

for partitions

$$
\pi: 0=t_{0}<t_{1}<t_{2}<\ldots
$$

See Stroock-Varadhan [55], Kunita [33] and Ichihara-Kunita [28, 29] for technical details.

Corollary 5.4. Suppose that the vector fields $A_{1}, \ldots, A_{r}$ are bracket-generating in the sense that condition (5.2) holds. Then

$$
\operatorname{supp} \mathbb{P}_{x}=C_{x}\left(\mathbb{R}_{+}, M\right) \quad \text { and } \operatorname{supp} \mathbb{P}_{t, x}=M .
$$

Proof. See Remark 5.2 above, as well as Stroock-Varadhan [55].

Remark 5.5. For stochastic representations of solutions to classical boundary value problems on a relatively compact open domain $D$ related to the Hörmander type operator

$$
L=A_{0}+\frac{1}{2} \sum_{i=1}^{r} A_{i}^{2}
$$

(see Sections 3.2 and 1.3) the following "finite exit time condition" had been crucial.

(a) Finite exit time condition. For each $x \in D$, the solution $X_{t}$ to $\operatorname{SDE}$ (5.1) with starting point $X_{0}=x$ exits $D$ in finite time almost surely.

In terms of the associated control system a sufficient condition for (a) to hold is given by the following escape condition.

(b) The domain $D$ is said to satisfy the escape condition if, for each $x \in D$, there is a control $u=u(\cdot) \in \mathcal{U}$ such that the path $t \mapsto \phi_{t}(x, u)$ in $C_{x}\left(\mathbb{R}_{+}, M\right)$ escapes from $\bar{D}$ (i.e., there exists a $T>0$ such that $\phi_{T}(x, u) \notin \bar{D}$ ).

The proof that the escape condition implies the finite exit time condition proceeds along the lines of the support theorem, see [54].

\section{Stochastic Flows of DiffeOMORPHisms}

We consider again an SDE on $M$ of the type

$$
d X=A(X) \circ d Z
$$

where $Z=\left(t, B^{1}, \ldots, B^{r}\right)$ with $B=\left(B^{1}, \ldots, B^{r}\right)$ a Brownian motion on $\mathbb{R}^{r}$. In equivalent form, Eq. (6.1) can be written as

$$
d X=A_{0}(X) d t+\sum_{i=1}^{r} A_{i}(X) \circ d B^{i}
$$

where the vector fields $A_{i}=A(\cdot) e_{i} \in \Gamma(T M)$ are taken with respect to the standard basis $\left(e_{0}, e_{1}, \ldots, e_{r}\right)$ of $\mathbb{R}^{r+1}$.

Let $\left(X_{t}(\cdot), \zeta(\cdot)\right)$ be the partial flow to

$$
L=A_{0}+\frac{1}{2} \sum_{i=1}^{r} A_{i}^{2},
$$

in the sense that for each $x \in M$, the process $X_{t}(x)$ has maximal lifetime $\zeta(x)$ and solves $\mathrm{SDE}$ (6.1). For $t \geq 0$ fixed, we then have the random set

$$
M_{t}(\omega)=\{x \in M: t<\zeta(x)(\omega)\}, \quad \omega \in \Omega .
$$

Theorem 6.1. The following assertions hold $\mathbb{P}$-almost surely (in $\omega \in \Omega$ ): 
i) $M_{t}(\omega)$ is an open subset of $M$ for each $t \geq 0$, i.e. $\zeta(\cdot)(\omega)$ is lower semicontinuous on $M$.

ii) For each $t \geq 0$, the map

$$
X_{t}(\cdot)(\omega): M_{t}(\omega) \longrightarrow R_{t}(\omega)
$$

is a diffeomorphism onto an open subset $R_{t}(\omega)$ of $M$.

iii) The path map $s \longmapsto X_{s}(\cdot)(\omega)$ is continuous from $[0, t]$ into $C^{\infty}\left(M_{t}(\omega), M\right)$ with its $C^{\infty}$-topology.

Proof. See Kunita's theory of stochastic flows [36].

Remark 6.2. Under "mild" growth conditions (see [36] for precise statements) on the vector fields $A_{0}, \ldots, A_{r}$ and their derivatives (which are trivially fulfilled if $M$ is compact), we have almost surely

$$
X_{t}(\cdot) \in \operatorname{Diff}(M) \text { for all } t .
$$

\subsection{Tangent flows and pullback of vector fields under stochastic flows.}

Proposition 6.3. In the situation of a partial flow to the SDE

$$
d X=\sum_{i=0}^{r} A_{i}(X) \circ d Z^{i}
$$

we consider the "tangent flow" $X_{t *}:=T X_{t}$, defined as the differential of the map $x \mapsto X_{t}(x)$,

$$
T_{x} M \rightarrow T_{X_{t}(x)} M, \quad v \longmapsto X_{t *} v, \quad x \in M_{t}(\omega) .
$$

The tangent map

$$
U_{t}:=X_{t *}
$$

solves the (formally) differentiated $\mathrm{SDE}(6.3)$, i.e.,

$$
d U=\sum_{i=0}^{r}\left(D A_{i}\right)_{X} U \circ d Z^{i}
$$

where $\left(D A_{i}\right)_{X}=T_{X} A_{i} \equiv T_{\pi(U)} A_{i}$. In addition, the inverse tangent flow $U_{t}^{\prime}=X_{t *}^{-1}$ solves the $\mathrm{SDE}$

$$
d U^{\prime}=-\sum_{i=0}^{r} U^{\prime}\left(D A_{i}\right)_{X} \circ d Z^{i} .
$$

Proof. These are standard formulas in the theory of SDEs and are checked in a straight-forward way using Stochastic Calculus, see [35, 36].

We now come to a crucial notion, the pullback of a vector field $V$ on $M$ under a stochastic flow $x \mapsto X_{t}(x)$. More precisely, for $V \in \Gamma(T M)$ we consider the (random) vector field $X_{t *}^{-1} V$ on $M_{t}$ defined as

$$
\left(X_{t *}^{-1} V\right)_{x}=\left(T_{x} X_{t}\right)^{-1} V_{X_{t}(x)} \in T_{x} M, \quad x \in M_{t} .
$$

In other words, we have

$$
\left(X_{t *}^{-1} V\right)(f)=V\left(f \circ X_{t}^{-1}\right) \circ X_{t}, \quad f \in C^{\infty}(M) .
$$

Lemma 6.4. The pullback vector field $X_{t *}^{-1} V$ satisfies the equation

$$
d\left(X_{t *}^{-1} V\right)=\sum_{i=0}^{r} X_{t *}^{-1}\left[A_{i}, V\right] \circ d Z_{t}^{i} .
$$


In the special form of $\mathrm{SDE}(6.2)$ this means

$$
d\left(X_{t *}^{-1} V\right)=X_{t *}^{-1}\left[A_{0}, V\right] d t+\sum_{i=1}^{r} X_{t *}^{-1}\left[A_{i}, V\right] \circ d B_{t}^{i} .
$$

Proof. For instance, see [34] Section 5.

Corollary 6.5. Suppose that the vector field $V$ commutes with $A_{0}, \ldots, A_{r}$. Then we have $X_{t *}^{-1} V=V$.

Remark 6.6. There are analogous formulas for the push-forward vector fields $X_{t *} V$ on $R_{t}$, e.g.,

$$
d\left(X_{t *} V\right)=\sum_{i=0}^{r}\left[X_{t *} A_{i}, V\right] \circ d Z_{t}^{i}
$$

resp.

$$
d\left(X_{t *} V\right)=\left[X_{t *} A_{0}, V\right] d t+\sum_{i=1}^{r}\left[X_{t *} A_{i}, V\right] \circ d B_{t}^{i} .
$$

\subsection{Malliavin's covariance matrix.}

Definition 6.7 (Malliavin's covariance matrix). Suppose that an SDE of the type

$$
d X=A_{0}(X) d t+\sum_{i=1}^{r} A_{i}(X) \circ d B^{i}
$$

is given. For $t>0$, the tensor

$$
C_{t}(x)=\sum_{i=1}^{r} \int_{0}^{t}\left(X_{s *}^{-1} A_{i}\right)_{x} \otimes\left(X_{s *}^{-1} A_{i}\right)_{x} d s \in T_{x} M \otimes T_{x} M, \quad x \in M_{t},
$$

defines a smooth (random) section of the bundle $T M \otimes T M$ over $M_{t}$. This section is usually called Malliavin's covariance matrix.

Malliavin's covariance matrix is at the heart of the so-called Malliavin Calculus, also known as Stochastic Calculus of Variations [47, 50]. In the sequel we use different notions of writing Malliavin's covariance matrix (6.6).

Notation 6.8. Putting together the diffusion vector fields $A_{1}, \ldots, A_{r}$ to a bundle map $A: M \times \mathbb{R}^{r} \rightarrow T M$ over $M$, we have

$$
\left(X_{s *}^{-1} A\right)_{x}: \mathbb{R}^{r} \rightarrow T_{x} M, \quad z \mapsto \sum_{i=1}^{r}\left(X_{s *}^{-1} A_{i}\right)_{x} z^{i} .
$$

(Note that the drift vector field $A_{0}$ is not included). Considering the dual map to Eq. (6.7),

$$
\left(X_{s *}^{-1} A\right)_{x}^{*}: T_{x}^{*} M \longrightarrow\left(\mathbb{R}^{r}\right)^{*} \equiv \mathbb{R}^{r},
$$

we may read Malliavin's covariance matrix (6.6) as

$$
C_{t}(x)=\int_{0}^{t}\left(X_{s *}^{-1} A\right)_{x}\left(X_{s *}^{-1} A\right)_{x}^{*} d s \in \operatorname{Hom}\left(T_{x} M, T_{x} M\right), \quad x \in M_{t} .
$$

Example 6.9. On $\mathbb{R}^{2}$ consider the SDE

$$
d X_{t}=A_{0}\left(X_{t}\right) d t+A_{1}\left(X_{t}\right) \circ d B_{t}^{1}, \quad X_{0}=x=\left(x^{1}, x^{2}\right),
$$

where $A_{0}=x^{1} \frac{\partial}{\partial x^{2}}$ and $A_{1}=\frac{\partial}{\partial x^{1}}$. Obviously $\operatorname{SDE}(6.8)$ writes as

$$
d X_{t}^{1}=d B_{t}^{1}, \quad d X_{t}^{2}=X_{t}^{1} d t, \quad\left(X_{0}^{1}, X_{0}^{2}\right)=\left(x^{1}, x^{2}\right),
$$


and so we have an explicit expression for the solution as

$$
\left\{\begin{array}{l}
X_{t}^{1}=x^{1}+B_{t}^{1} \\
X_{t}^{2}=x^{2}+x^{1} t+\int_{0}^{t} B_{s}^{1} d s .
\end{array}\right.
$$

Thus

$$
X_{t *}=\left(\begin{array}{cc}
1 & 0 \\
t & 1
\end{array}\right) \quad \text { and } \quad X_{t *}^{-1}=\left(\begin{array}{cc}
1 & 0 \\
-t & 1
\end{array}\right) .
$$

For Malliavin's covariance matrix we get

$$
C_{t}(x)=\left(\begin{array}{cc}
t & -t^{2} / 2 \\
-t^{2} / 2 & t^{3} / 3
\end{array}\right)
$$

Note that $C_{t}(x)$ is independent of $x$ and invertible for $t>0$. SDE (6.8) is degenerate in the sense that $A_{1}$ does not $\operatorname{span} T_{x} \mathbb{R}^{2}$, but observe that $\left[A_{0}, A_{1}\right]=\frac{\partial}{\partial x^{2}}$. It is easy to see that the random vector $\left(X_{t}^{1}, X_{t}^{2}\right)$ has a Gaussian distribution with covariance

$$
\left(\begin{array}{cc}
t & t^{2} / 2 \\
t^{2} / 2 & t^{3} / 3
\end{array}\right)
$$

For $t>0$ the covariance is non-singular, and hence $\left(X_{t}^{1}, X_{t}^{2}\right)$ has a smooth Gaussian density function with respect to the 2-dimensional Lebesgue measure.

\section{StochastiC FLOWS AND HYPOELliptiCITY}

The purpose of this section is to sketch a probabilistic proof of Hörmander's hypoellipticity theorem. We follow some of the arguments in Bismut [14].

Consider a second order PDO in Hörmander form

$$
L=A_{0}+\frac{1}{2} \sum_{i=1}^{r} A_{i}^{2}
$$

on a differentiable manifold $M$ with smooth vector fields $A_{0}, \ldots, A_{r}$. For simplicity, we assume again that all vector fields of the form

$$
A_{0}+\frac{1}{2} \sum_{i=1}^{r} A_{i} u^{i}, \quad u \in \mathbb{R}^{r}
$$

are complete.

We denote by $\mathscr{D}^{\prime}(M)$ the space of distributions on $M$. Recall that an operator $L$ of the type (7.1) is called hypoelliptic if $u \in \mathscr{D}^{\prime}(M)$ and $L u \mid U \in C^{\infty}(U)$ where $U \subset M$ is open, implies that $u \mid U \in C^{\infty}(U)$.

Our goal be to show hypoellipticity of the operator (7.1) under a certain Hörmandertype non-degeneracy.

7.1. Hypoellipticity under Hörmander conditions. Consider the following two canonical measures on $M$ :

$$
\begin{aligned}
\mathbb{P}_{t}(x, d y) & :=\mathbb{P}\left\{X_{t}(x) \in d y\right\}, \quad \text { and } \\
G_{\lambda}(x, d y) & :=\int_{0}^{\infty} e^{-\lambda t} \mathbb{P}\left\{X_{t}(x) \in d y\right\} d t, \quad \lambda>0 .
\end{aligned}
$$

Remark 7.1. In Section 3 these measures have been used for stochastic representation formulas of classical PDEs. 
i) Recall that every bounded solution $u(t, x)$ to the initial value problem

$$
\frac{\partial}{\partial t} u=L u,\left.\quad u\right|_{t=0}=f
$$

can be represented as

$$
u(t, x)=\int P_{t}(x, d y) f(y)=E\left[f \circ X_{t}(x)\right] .
$$

ii) According to the Feynman-Kac formula (3.5), solutions to

$$
(\lambda-L) u=f
$$

have a representation as

$$
u(x)=\int G_{\lambda}(x, d y) f(y), \quad x \in M .
$$

In this sense, the operator $G_{\lambda}$ defines the inverse to $\lambda-L$, formally $G_{\lambda}=$ $(\lambda-L)^{-1}$.

Choosing a smooth volume measure vol on $M$, we now come to the following fundamental question.

Problem 7.2. When do measures like $P_{t}(x, \cdot)$ or $G_{\lambda}(x, \cdot)$ have densities with respect to vol?

Definition 7.3. To the vector fields $A_{0}, \ldots, A_{r}$ defining the operator

$$
L=A_{0}+\frac{1}{2} \sum_{i=1}^{r} A_{i}^{2}
$$

we associate several important Lie algebras $[28,29,5]$.

- On $M$ :

$$
\begin{aligned}
\mathscr{L} & :=\operatorname{Lie}\left(A_{0}, A_{1}, \ldots, A_{r}\right) \\
\mathscr{B} & :=\operatorname{Lie}\left(A_{1}, \ldots, A_{r}\right) \\
\mathscr{I} & :=\text { ideal in } \mathscr{L} \text { generated by } \mathscr{B} .
\end{aligned}
$$

- On $M \times \mathbb{R}:$

$$
\hat{\mathscr{L}}:=\operatorname{Lie}\left(A_{0}+\frac{\partial}{\partial t}, A_{1}, \ldots, A_{r}\right) .
$$

By definition we have $\mathscr{B} \subset \mathscr{I} \subset \mathscr{L}$.

In terms of these Lie algebras we consider the following Hörmander conditions $(n=\operatorname{dim} M)$ :

$\left(\mathrm{H}_{0}\right) \quad \operatorname{dim} \mathscr{L}(x)=n, \quad$ at each point $x$ of $M$,

$\left(\mathrm{H}_{1}\right) \quad \operatorname{dim} \hat{\mathscr{L}}(t, x)=n+1, \quad$ at each point $(x, t)$ of $M \times \mathbb{R}$.

Hypothesis $\left(\mathrm{H}_{0}\right)$ means that

$$
\operatorname{Lie}\left(A_{0}, A_{1}, \ldots, A_{r}\right)(x)=T_{x} M \quad \text { for all } x \in M,
$$

whereas $\left(\mathrm{H}_{1}\right)$ is equivalent to

$\operatorname{Lie}\left(A_{1}, \ldots, A_{r},\left[A_{i}, A_{j}\right]_{0 \leq i, j \leq r},\left[A_{i},\left[A_{j}, A_{k}\right]\right]_{0 \leq i, j, k \leq r}, \ldots\right)(x)=T_{x} M \quad$ for all $x \in M$.

This last condition can be equivalently stated as

$$
\operatorname{dim} \mathscr{I}(x)=n, \quad \text { at each point } x \text { of } M .
$$

Theorem 7.4 (Hörmander (1967) [26]).

(1) Under hypothesis $\left(\mathrm{H}_{0}\right)$ the operator $L$ is hypoelliptic on $M$. 
(2) Under hypothesis $\left(\mathrm{H}_{1}\right)$ the space-time operator $L+\frac{\partial}{\partial t}$ hypoelliptic on $M \times \mathbb{R}$.

It can be shown that Hörmander's condition $\left(\mathrm{H}_{0}\right)$ is necessary for hypoellipticity for operators $L$ with analytic coefficients. Such is not the case for smooth vector fields $A_{0}, A_{1}, \ldots, A_{r}$. The stochastic approach allows to derive sharper criterions for hypoellipticity that allow Hrmander's condition to fail on tiny tiny subsets of $M$, see for instance [11].

Remark 7.5. Let $L^{*}$ be the formal adjoint operator to $L$ (with respect to the chosen volume measure). It can be written as

$$
L^{*}=\frac{1}{2} \sum_{i=1}^{r} A_{i}^{2}+\tilde{A}_{0}+a \quad \text { where } A_{0}+\tilde{A}_{0} \in \mathscr{B} .
$$

Thus we have the following equivalences:

(i) $L$ satisfies $\left(\mathrm{H}_{0}\right)$ if and only if $L^{*}-a$ satisfies $\left(\mathrm{H}_{0}\right)$;

(ii) $L+\frac{\partial}{\partial t}$ satisfies $\left(\mathrm{H}_{1}\right)$ if and only if $L^{*}+\frac{\partial}{\partial t}-a$ satisfies $\left(\mathrm{H}_{1}\right)$.

Corollary 7.6. We may consider the measures $G_{\lambda}(x, \cdot)$ and $P_{t}(x, \cdot)$ as distributions as follows:

$$
G_{\lambda}(x, d y) \in \mathscr{D}^{\prime}(M \times M), \quad P_{t}(x, d y) \in \mathscr{D}^{\prime}(] 0, t[\times M \times M) .
$$

Denoting by $\Lambda$ the diagonal in $M \times M$, the following equations hold in the weak sense:

$$
\begin{aligned}
& \left(\lambda-L_{x}\right) G_{\lambda}=\mathbb{1}_{\Lambda} \\
& \left(\lambda-L_{y}^{*}\right) G_{\Lambda}=\mathbb{1}_{\Lambda} \\
& \left(\frac{\partial}{\partial t}-L_{x}\right) P_{t}(x, d y)=0 \\
& \left(\frac{\partial}{\partial t}-L_{y}^{*}\right) P_{t}(x, d y)=0 .
\end{aligned}
$$

By means of Hörmander's Theorem 7.4 we obtain:

(a) Suppose that condition $\left(\mathrm{H}_{0}\right)$ holds. Then the operator $L$ is hypoelliptic and there exists a function $g_{\lambda} \in C^{\infty}((M \times M) \backslash \Delta)$ such that

$$
G_{\lambda}(x, d y)=g_{\lambda}(x, y) \operatorname{vol}(d y) .
$$

(b) Suppose that condition $\left(\mathrm{H}_{1}\right)$ holds. Then the operator $\frac{\partial}{\partial t}-L$ is hypoelliptic and there exists a function $p_{t}(x, y)$ in $C^{\infty}(] 0, \infty[\times M \times M)$ such that

$$
P_{t}(x, d y)=p_{t}(x, y) \operatorname{vol}(d y)
$$

In the sequel, to avoid technical problems, we assume $A_{0}, A_{1}, \ldots, A_{r} \in \Gamma(T M)$ along with their derivatives to satisfy some growth conditions. Such conditions will be necessary below to make some quantities well-defined. To this end we choose a Riemannian metric on $M$; the volume form $\operatorname{vol}(d y)$ will be taken with respect to this metric.

Standing Hypothesis. Assume that the vector fields $A_{0}, A_{1}, \ldots, A_{r}$ are smooth with bounded derivatives of all orders.

Remark 7.7. This hypothesis is far from being necessary, but it guarantees that solutions to the Eqs. (6.3), (6.4) and (6.5) lie in any $L^{p}$ space $(1 \leq p<\infty)$ uniformly over compact time intervals.

The following theorem gives a probabilistic approach to Hörmander's hypoellipticity theorem, see $[14,38,39,40,49,50]$, as well as Malliavin's original work [44, $45,46]$. 
Theorem 7.8. For $x \in M$, let $X \equiv X$. $(x)$ be solution to the Stratonovich SDE

$$
d X=A_{0}(X) d t+\sum_{i=1}^{r} A_{i}(X) \circ d B^{i}, \quad \text { with initial condition } X_{0}=x .
$$

Suppose that for each $t>0$ the following two conditions hold true:

i) The bilinear form

$$
C_{t}(x):=\sum_{i=1}^{r} \int_{0}^{t}\left(X_{s *}^{-1} A_{i}\right)_{x} \otimes\left(X_{s *}^{-1} A_{i}\right)_{x} d s \quad \text { on } T_{x}^{*} M \otimes T_{x}^{*} M
$$

is almost surely non-degenerate.

ii) In terms of the inner product on $T_{x} M$ and reading $C_{t}(x) \in \operatorname{Hom}\left(T_{x} M, T_{x} M\right)$, we have

$$
\left|C_{t}(x)^{-1}\right| \in L^{p} \quad \text { for each } p \geq 1 .
$$

Then there exists a function $p_{t}(x, y)$ in $C^{\infty}(] 0, t[\times M \times M)$ such that

$$
P_{t}(x, d y)=p_{t}(x, y) \operatorname{vol}(d y) \text {. }
$$

Remark 7.9. Thus proving Hörmander's parabolic result will come down to show that under hypothesis $\left(\mathrm{H}_{1}\right)$ the conditions (i) and (ii) of Theorem 7.8 are satisfied. We shall sketch the essential steps of the proof in the remainder of this section.

The idea underlying the probabilistic approach is the following. The measure $P_{t}(x, d y)$ is the image of the Wiener measure under the mapping $X_{t}(x): \omega \mapsto$ $X_{t}(x, \omega)$. Since Wiener measure has a well-understood analytic structure, if this map were "smooth" then regularity properties of $P_{t}(x, d y)$ could be obtained by integration by parts on Wiener space. The goal of Malliavin Calculus is to overcome the difficulty that the map $X_{t}(x)$ however is most pathological from the standpoint of classical analysis or standard calculus. See [37, 25, 10] for survey articles along these lines.

Theorem 7.10. Suppose that $\left(\mathrm{H}_{1}\right)$ holds true, i.e.

$\left(\mathrm{H}_{1}\right)$

$\operatorname{dim} \operatorname{Lie}\left(A_{1}, \ldots, A_{r},\left[A_{i}, A_{j}\right]_{0 \leq i, j \leq r},\left[A_{i},\left[A_{j}, A_{k}\right]\right]_{0 \leq i, j, k \leq r}, \ldots\right)(x)=n \quad$ for each $x \in M$.

Then, for each $x \in M$ and each $t>0$, almost surely,

$$
C_{t}(x)=\sum_{i=1}^{r} \int_{0}^{t}\left(X_{s *}^{-1} A_{i}\right)_{x} \otimes\left(X_{s *}^{-1} A_{i}\right)_{x} d s \in T_{x} M \otimes T_{x} M
$$

defines a non-degenerate symmetric bilinear form on $T_{x}^{*} M$.

Proof. We fix $x \in M$ and let

$$
\begin{aligned}
\mathscr{G}_{s} & :=\operatorname{span}\left(\left(X_{s *}^{-1} A_{i}\right)_{x}: i=1, \ldots, r\right) \subset T_{x} M, \\
\mathscr{U}_{t} & :=\operatorname{span}\left(\bigcup_{s \leq t} \mathscr{G}_{s}\right), \quad t>0, \\
\mathscr{U}_{t}^{+} & :=\bigcap_{s>t} \mathscr{U}_{s} .
\end{aligned}
$$

Then (by the 0/1-law of Blumenthal) $\mathscr{U}_{0}^{+}$is almost surely a fixed (deterministic) linear subspace of $T_{x} M$. We have to show that almost surely

$$
\mathscr{U}_{0}^{+}=T_{x} M .
$$

Suppose that $\mathscr{U}_{0}^{+} \varsubsetneqq T_{x} M$. Then the stopping time

$$
\sigma:=\inf \left\{t>0: \mathscr{U}_{t} \neq \mathscr{U}_{0}^{+}\right\}
$$


is almost surely strictly positive. Let $\xi \in T_{x} M$ such that $\xi \perp \mathscr{U}_{0}^{+}$. Then, in particular, $\xi \perp \mathscr{U}_{t}$ for all $t<\sigma$. In other words, we have for each $i=1, \ldots, r$,

$$
\left\langle\xi,\left(X_{t *}^{-1} A_{i}\right)_{x}\right\rangle=0, \quad \text { for any } t<\sigma .
$$

However, for any $V \in \Gamma(T M)$, we know that

$$
\begin{aligned}
d\left(X_{s *}^{-1} V\right)_{x} & =\left(X_{s *}^{-1}\left[A_{0}, V\right]\right)_{x} d s+\sum_{j=1}^{r}\left(X_{s *}^{-1}\left[A_{j}, V\right]\right)_{x} \circ d B_{s}^{j} \\
& =\left(X_{s *}^{-1}\left[A_{0}, V\right]\right)_{x} d s+\sum_{j=1}^{r}\left(X_{s *}^{-1}\left[A_{j}, V\right]\right)_{x} d B_{s}^{j}+\sum_{j=1}^{r}\left(X_{s *}^{-1}\left[A_{j},\left[A_{j}, V\right]\right]\right)_{x} d s
\end{aligned}
$$

Thus, taking $V=A_{i}$ where $1 \leq i \leq r$, we have for $t<\sigma$,

$$
\begin{aligned}
\underbrace{\left\langle\xi,\left(X_{t *}^{-1} A_{i}\right)_{x}\right\rangle}_{=0}=\underbrace{\left\langle\xi, A_{i}(x)\right\rangle}_{=0}+\int_{0}^{t}\left\langle\xi,\left(X_{s *}^{-1}\left[A_{0}, A_{i}\right]\right)_{x}\right\rangle d s & +\sum_{j=1}^{r} \int_{0}^{t}\left\langle\xi,\left(X_{s *}^{-1}\left[A_{j}, A_{i}\right]\right)_{x}\right\rangle d B_{s}^{j} \\
& +\sum_{j=1}^{r} \int_{0}^{t}\left\langle\xi,\left(X_{s *}^{-1}\left[A_{j},\left[A_{j}, A_{i}\right]\right]\right)_{x}\right\rangle d s .
\end{aligned}
$$

By uniqueness of the Doob-Meyer decomposition, canceling the martingale part in Eq. (7.6), we first obtain

$$
\left\langle\xi,\left(X_{s *}^{-1}\left[A_{j}, A_{i}\right]\right)_{x}\right\rangle=0, \quad \text { for } 1 \leq i, j \leq r \text { and } s<\sigma .
$$

By repeating the above calculation with $\left[A_{j}, A_{i}\right]$ instead of $A_{i}$ we get

$$
\left\langle\xi,\left(X_{s *}^{-1}\left[A_{j},\left[A_{j}, A_{i}\right]\right]\right)_{x}\right\rangle=0, \quad \text { for } 1 \leq i, j \leq r \text { and } s<\sigma .
$$

This allows to cancel the bounded variation part in Eq. (7.6) which gives in addition

$$
\left\langle\xi,\left(X_{s *}^{-1}\left[A_{0}, A_{i}\right]\right)_{x}\right\rangle=0, \quad \text { for } 1 \leq i \leq r \text { and } s<\sigma .
$$

By iteration, we see that if $A_{[I]}$ is any of the brackets appearing in $\left(\mathrm{H}_{1}\right)$, i.e.

$$
A_{[I]} \in \operatorname{Lie}\left(A_{1}, \ldots, A_{r},\left[A_{i}, A_{j}\right]_{0 \leq i, j \leq r},\left[A_{i},\left[A_{j}, A_{k}\right]\right]_{0 \leq i, j, k \leq r}, \ldots\right)
$$

then

$$
\left\langle\xi,\left(X_{s *}^{-1} A_{[I]}\right)_{x}\right\rangle=0, \quad s<\sigma .
$$

In particular, by taking $s=0$, we find that

$$
\left\langle\xi,\left(A_{[I]}\right)_{x}\right\rangle=0
$$

But, since according to $\left(\mathrm{H}_{1}\right)$,

$$
\operatorname{Lie}\left(A_{1}, \ldots, A_{r},\left[A_{i}, A_{j}\right]_{0 \leq i, j \leq r},\left[A_{i},\left[A_{j}, A_{k}\right]\right]_{0 \leq i, j, k \leq r}, \ldots\right)(x)=T_{x} M,
$$

we conclude $\xi=0$.

In the sequel, we want to sketch the proof that, for given $x \in M$ and $t>0$,

$$
P_{t}(x, d y)=p_{t}(x, y) \operatorname{vol}(d y)
$$

where $p_{t}(x, \cdot) \in C^{\infty}(M)$. This is the essential part in the stochastic proof of Corollary $7.6(\mathrm{~b})$. To this end, we have to show that $\mu=P_{t}(x, d y)$ as a distribution is sufficiently smooth. This means that we have to find estimates for the distributional derivatives of $\mu$.

Lemma 7.11. Let $\mu$ be a probability measure on a manifold $M(\operatorname{dim} M=n)$ such that

$$
\left|\left\langle f, D^{(\alpha)} \mu\right\rangle\right| \leq C_{\alpha}\|f\|_{\infty} \quad \text { for all } \alpha \in \mathbb{N}^{n} \text { and } f \in C_{c}^{\infty}(M) .
$$

Then $\mu(d y)=\rho(y) \operatorname{vol}(d y)$ with $\rho \in L^{1}(d y) \cap C^{\infty}(M)$. 
Hence to achieve (7.7) for the measure $\mu(d y)=P_{t}(x, d y)$, we have to show that

$$
\left|\mathbb{E}\left[\left(D^{(\alpha)} f\right)\left(X_{t}(x)\right)\right]\right| \leq C_{\alpha}\|f\|_{\infty} \quad \forall \alpha \in \mathbb{N}^{n} .
$$

7.2. Girsanov's theorem. In the sequel we shall use a basic fact from Stochastic Analysis. This is a special case of Girsanov's theorem [52] which specifies how to remove a drift by change of measure.

Theorem 7.12 (Girsanov). Let $B$ be a standard Brownian motion on $\mathbb{R}^{r}$ and let $u_{t}$ be a continuous adapted process taking values in $\mathbb{R}^{r}$ as well, such that

$$
\mathbb{E}\left[\exp \left(\frac{1}{2} \int_{0}^{t}\left|u_{s}\right|^{2} d s\right)\right]<\infty .
$$

Consider the Brownian motion with drift $\hat{B}$ defined as

$$
d \hat{B}_{t}:=d B_{t}+u_{t} d t
$$

Then, if $B$ is a Brownian motion on $\mathbb{R}^{r}$ with respect to $\mathbb{P}$, then $\hat{B}$ is a Brownian motion on $\mathbb{R}^{r}$ with respect to $\hat{\mathbb{P}}$ where the new probability measure $\hat{\mathbb{P}}$ is given by

$$
\left.\frac{d \hat{\mathbb{P}}}{d \mathbb{P}}\right|_{\mathscr{F}_{t}}=\exp \left(-\int_{0}^{t} u_{s} d B_{s}-\frac{1}{2} \int_{0}^{t}\left|u_{s}\right|^{2} d s\right)
$$

Hence, defining

$$
G_{t}:=\exp \left(-\int_{0}^{t} u_{s} d B_{s}-\frac{1}{2} \int_{0}^{t}\left|u_{s}\right|^{2} d s\right)
$$

we have

$$
d \hat{\mathbb{P}}=G_{t} d \mathbb{P} \quad \text { on } \mathscr{F}_{t} .
$$

In particular, for any measurable functional $F$ on path space, we conclude that

$$
\mathbb{E}_{\mathbb{P}}[F(B .)]=\mathbb{E}_{\hat{\mathbb{P}}}\left[F\left(\hat{B}_{.}\right)\right] .
$$

Eq. (7.8) specifies us how a perturbation of a standard Brownian motion by an additive drift can be compensated via a change of measure.

7.3. Elementary stochastic calculus of variations. We fix a point $x \in M$ and consider $u_{s}=\lambda a_{s}$ where $\lambda \in T_{x}^{*} M$ and where $a_{s}$ is a continuous adapted process taking values in

$$
T_{x} M \otimes\left(\mathbb{R}^{r}\right)^{*} \equiv T_{x} M \otimes \mathbb{R}^{r}
$$

such that

$$
\mathbb{E}\left[\exp \left(\frac{1}{2} \int_{0}^{t}\left|\lambda a_{s}\right|^{2} d s\right)\right]<\infty
$$

for all $\lambda$ in a small neighbourhood $U$ about 0 .

In the SDE (7.4) defining the stochastic flow $X$, we add a drift to the driving Brownian motion $B$,

$$
d B_{t}^{\lambda}:=d B_{t}+\lambda a_{t} d t
$$

and compensate this perturbation by changing the measure from $\mathbb{P}$ to $\mathbb{P}^{\lambda}$,

$$
\mathbb{P}^{\lambda}\left|\mathscr{F}_{t}=G_{t}^{\lambda} \cdot \mathbb{P}\right| \mathscr{F}_{t}
$$

where

$$
G_{t}^{\lambda}=\exp \left(-\int_{0}^{t} \lambda a_{s} d B_{s}-\frac{1}{2} \int_{0}^{t}\left|\lambda a_{s}\right|^{2} d s\right) .
$$

We denote by $X_{\text {. }}^{\lambda}(x)$ the solution to $\operatorname{SDE}$ (7.4) when driven by $B_{t}^{\lambda}$ instead of $B_{t}$.

By Girsanov's theorem, we may conclude that

$$
\mathbb{E}\left[f\left(X_{t}^{\lambda}(x)\right) g\left(B_{.}^{\lambda}\right) G_{t}^{\lambda}\right] \quad \text { is independent of } \lambda .
$$


Here $f$ is a smooth function on $M$ and $g$ is a functional of $B_{.}^{\lambda} \mid[0, t]$ such that $g\left(B_{.}^{\lambda}\right)$ is differentiable in $\lambda$. The explicit form of $g$ will be determined later.

We may assume that $\operatorname{supp}(f)$ lies in a chart of $M$; then we write $\left(D_{i} f\right)(x):=$ $(d f)_{x} e_{i}$. Also, since $x \in M$ fixed, we identify $T_{x} M$ with $\mathbb{R}^{n}$.

From Eq. (7.9) we know that

which gives

$$
\left.\frac{\partial}{\partial \lambda_{k}}\right|_{\lambda=0} \mathbb{E}\left[f\left(X_{t}^{\lambda}(x)\right) g\left(B_{.}^{\lambda}\right) G_{t}^{\lambda}\right]=0
$$

We write

$$
\begin{array}{r}
\mathbb{E}\left[\sum_{i}\left(D_{i} f\right)\left(X_{t}(x)\right)\left(\left.\frac{\partial}{\partial \lambda_{k}}\right|_{\lambda=0} X_{t}^{\lambda}(x)\right)^{i} g\left(B_{.}\right)\right] \\
=-\mathbb{E}\left[\left.f\left(X_{t}(x)\right) \frac{\partial}{\partial \lambda_{k}}\right|_{\lambda=0}\left(g\left(B_{.}^{\lambda}\right) G_{t}^{\lambda}\right)\right] .
\end{array}
$$

It is easily checked that

$$
\left(\left.\frac{\partial}{\partial \lambda_{k}}\right|_{\lambda=0} X_{t}^{\lambda}(x)\right)^{i}=\left(\partial X_{t}(x)\right)_{i k} .
$$

$$
\left.\partial X_{t}(x) \equiv \frac{\partial}{\partial \lambda}\right|_{\lambda=0} X_{t}^{\lambda}(x)=X_{t *} \int_{0}^{t}\left(X_{t *}^{-1} A\right)_{x} a_{s} d s \in \operatorname{Hom}\left(T_{x} M, T_{x} M\right) .
$$

Thus, if we take

$$
a_{s}:=\left(X_{s *}^{-1} A\right)_{x}^{*}: T_{x}^{*} M \rightarrow \mathbb{R}^{r},
$$

then

Finally, taking

$$
\partial X_{t}(x)=X_{t *} C_{t}(x)
$$

$$
g\left(B_{.}^{\lambda}\right):=\left(C_{t}^{\lambda}(x)^{-1}\left(X_{t *}^{\lambda}\right)^{-1}\right)_{k j} \gamma\left(B_{.}^{\lambda}\right)
$$

where $\gamma\left(B_{.}^{\lambda}\right)$ is specified later, and then summing over $k$, we obtain

$$
\mathbb{E}\left[\left(D_{j} f\right)\left(X_{t}(x)\right) \gamma(B .)\right]=-\mathbb{E}[f\left(X_{t}(x)\right) \underbrace{\left.\sum_{k} \frac{\partial}{\partial \lambda_{k}}\right|_{\lambda=0}\left(C_{t}^{\lambda}(x)^{-1}\left(X_{t *}^{\lambda}\right)^{-1}\right)_{k j} \gamma\left(B_{.}^{\lambda}\right) G_{t}^{\lambda}}_{=: \mathscr{H}_{j}(\gamma)}] .
$$

By iteration, this shows that

$$
\mathbb{E}\left[\left(D_{i} D_{j} D_{k} \ldots f\right)\left(X_{t}(x)\right)\right]=-\mathbb{E}\left[f\left(X_{t}(x)\right)\left(\ldots \mathscr{H}_{k} \mathscr{H}_{j} \mathscr{H}_{i}\left(\mathbb{1}_{M}\right)\right)\right] .
$$

From Eq. (7.10) we get the crucial estimate

$$
\left|\mathbb{E}\left[\left(D_{i} D_{j} D_{k} \ldots f\right)\left(X_{t}(x)\right)\right]\right| \leq\|f\|_{\infty} \times\left\|\ldots \mathscr{H}_{k} \mathscr{H}_{j} \mathscr{H}_{i}\left(\mathbb{1}_{M}\right)\right\|_{1}
$$

where $\mathbb{1}_{M}$ denotes the function on $M$ which is identically equal to 1 . Hence, to conclude, it remains to show that

$$
\left\|\ldots \mathscr{H}_{k} \mathscr{H}_{j} \mathscr{H}_{i}\left(\mathbb{1}_{M}\right)\right\|_{1}<\infty
$$

for arbitrary indices $1 \leq i, j, k, \ldots \leq n$.

The terms appearing in the norm in (7.12) can easily be worked out explicitly by using formulas like

$$
\begin{aligned}
\left.\frac{\partial}{\partial \lambda_{k}}\right|_{\lambda=0} C_{t}^{\lambda}(x)^{-1} & =-\left.C_{t}(x)^{-1} \frac{\partial}{\partial \lambda_{k}}\right|_{\lambda=0} C_{t}^{\lambda} C_{t}^{\lambda}(x)^{-1} \\
\left.\frac{\partial}{\partial \lambda_{k}}\right|_{\lambda=0} G_{t}^{\lambda} & =-\left(\int_{0}^{t}\left(X_{s *}^{-1} A\right)_{x} d B_{s}\right)_{k} .
\end{aligned}
$$


Note that apart from $C_{t}^{-1}(x)$ only polynomial expressions of quantities appear which lie in each $L^{p}$-space $(1 \leq p<\infty)$.

To conclude the proof of Theorem 7.8 the integrability condition (7.5) still needs to be verified. This requires some non-trivial technical estimates, see [39] for a detailed exposition, as well as the simplifications due to [49]. A unified treatment of these issues can be found in [50].

\section{Future PRospects}

Given a sub-Riemannian structure on a differentiable manifold $M$ we discussed the problem of defining a canonical sub-Laplacian $L=\Delta_{\mathcal{H}}$ on $M$, either as $L=$ $\operatorname{trace}_{\mathcal{H}} \nabla d f$ by choosing a metric partial connection on $\mathcal{H}$, or by endowing $M$ with a smooth volume measure and defining $L$ as the divergence of the horizontal gradient. Such sub-Laplacians have a representation in Hörmander form as

$$
L=A_{0}+\frac{1}{2} \sum_{i=1}^{r} A_{i}^{2}
$$

with vector fields $A_{0}, A_{1}, \ldots, A_{r} \in \Gamma(\mathcal{H}) \subset \Gamma(T M)$. Under the assumption that Hörmander's bracket-generating condition $\left(\mathrm{H}_{1}\right)$ is satisfied, existence of a smooth heat kernel $p_{t}(x, y)$ in $C^{\infty}(] 0, \infty[\times M \times M)$ is guaranteed,

$$
P_{t}(x, d y):=\mathbb{P}\left\{X_{t}(x) \in d y\right\}=p_{t}(x, y) \operatorname{vol}(d y),
$$

and probabilistic methods can be applied to investigate the asymptotics of $p_{t}(x, y)$ for small and large times. Heat kernel asymptotic expansion is well studied in Riemannian and sub-Riemannian geometry. Classical results of Gerard Ben Arous, Remi Léandre and others $[12,42,41]$ include such asymptotic expansion for the case of diagonal $p_{t}(x, x)$ and off-diagonal and off cut-locus $p_{t}(x, y)$; the on cut-locus case $p_{t}(x, y)$ is only understood up to the leading order [4]. For application of Malliavin Calculus in the study of heat kernel expansions see [58].

In terms of the $\Gamma$-operator

$$
\Gamma(f, g)=\frac{1}{2}(L(f g)-f L g-g L f), \quad f, g \in C^{\infty}(M),
$$

the Carnot-Carathéodory distance on $M$ is defined as

$$
\mathrm{d}_{\mathrm{CC}}(x, y):=\sup \left\{|f(x)-f(y)|: f \in C_{c}^{\infty}(M), \Gamma(f, f) \leq 1\right\} .
$$

Under the strong Hörmander condition,

$$
\operatorname{Lie}\left(A_{1}, \ldots, A_{r}\right)(x)=T_{x} M, \quad x \in M,
$$

the Carnot-Carathéodory distance is finite and (8.2) defines a metric structure on $M$.

As in Riemannian geometry a natural question is to investigate the radial process

$$
R_{t}:=\mathrm{d}_{\mathrm{CC}}\left(x_{0}, X_{t}(x)\right)
$$

for large times [27]. On a Riemannian manifold, by means of classical Laplacian comparison theorems, the speed of the radial process can be controlled by lower (Ricci) curvature bounds. Defining curvature in sub-Riemannian geometry however is an intriguing problem [1]. Up to now, for instance, no direct probabilistic proof for non-explosion in finite time of sub-Riemannian diffusion by controlling the radial process (8.3) under sub-Riemannian curvature bounds is known [21].

During the last years, several results have appeared, linking sub-Riemannian geometric invariants to properties of diffusions of corresponding second order operators and their heat semi-group, see $[6,7,22,23]$. These so-called curvature-dimension 
inequalities are based on a generalization of the $\Gamma_{2}$-calculus for sub-Riemannian manifolds introduced by Fabrice Baudoin and Nicola Garofalo [8].

Connections between the probabilistic behaviour of subelliptic diffusions and analytic properties of the corresponding heat semigroups, most directly expressed in functional inequalities, have attracted a lot of attention [17, 48, 43, 3]. For instance, denoting by $P_{t} f$ the (minimal) heat semigroup generated by

$$
L=A_{0}+\frac{1}{2} \sum_{i=1}^{r} A_{i}^{2}
$$

acting on bounded functions $f \in C^{\infty}(M)$, one seeks to find a constant $C$ such that

$$
\left|\nabla^{\text {hor }} P_{t} f\right|^{2} \leq C P_{t}\left|\nabla^{\text {hor }} f\right|^{2}
$$

holds pointwise for any $t>0$, see $[17,48]$. Note that the squared norm of the horizontal gradient $\nabla^{\text {hor }} f$ is given by

$$
\left|\nabla^{\text {hor }} f\right|^{2}=\sum_{i=1}^{r}\left(A_{i} f\right)^{2}
$$

Conversely, functional inequalities of the type as (8.4) can be used to deduce nonexplosion of the underlying diffusion $[9,21]$.

\section{REFERENCES}

1. Andrei Agrachev, Davide Barilari, and Luca Rizzi, Curvature: a variational approach, To appear in: Memoirs American Mathematical Society.

2. Andrei A. Agrachev and Yuri L. Sachkov, Control theory from the geometric viewpoint, Encyclopaedia of Mathematical Sciences, vol. 87, Springer-Verlag, Berlin, 2004, Control Theory and Optimization, II. MR 2062547 (2005b:93002)

3. Dominique Bakry, Fabrice Baudoin, Michel Bonnefont, and Djalil Chafaï, On gradient bounds for the heat kernel on the Heisenberg group, J. Funct. Anal. 255 (2008), no. 8, 1905-1938. MR 2462581 (2010m:35534)

4. Davide Barilari, Ugo Boscain, and Robert W. Neel, Small-time heat kernel asymptotics at the sub-Riemannian cut locus, J. Differential Geom. 92 (2012), no. 3, 373-416. MR 3005058

5. Fabrice Baudoin, An introduction to the geometry of stochastic flows, Imperial College Press, London, 2004. MR 2154760 (2006f:60003)

6. Fabrice Baudoin and Michel Bonnefont, Log-Sobolev inequalities for subelliptic operators satisfying a generalized curvature dimension inequality, J. Funct. Anal. 262 (2012), no. 6, 26462676. MR 2885961

7. Fabrice Baudoin, Michel Bonnefont, and Nicola Garofalo, A sub-Riemannian curvaturedimension inequality, volume doubling property and the Poincaré inequality, Math. Ann. 358 (2014), no. 3-4, 833-860. MR 3175142

8. Fabrice Baudoin and Nicola Garofalo, Curvature-dimension inequalities and Ricci lower bounds for sub-Riemannian manifolds with transverse symmetries, To appear in: Journal of the EMS.

9. Fabrice Baudoin and Jing Wang, Curvature dimension inequalities and subelliptic heat kernel gradient bounds on contact manifolds, Potential Anal. 40 (2014), no. 2, 163-193. MR 3152160

10. Denis Bell, The Malliavin calculus and hypoelliptic differential operators, Infin. Dimens. Anal. Quantum Probab. Relat. Top. 18 (2015), no. 1, 1550001, 24. MR 3324717

11. Denis R. Bell and Salah Eldin A. Mohammed, An extension of Hörmander's theorem for infinitely degenerate second-order operators, Duke Math. J. 78 (1995), no. 3, 453-475. MR 1334203 (96g:35034)

12. Gérard Ben Arous, Développement asymptotique du noyau de la chaleur hypoelliptique hors du cut-locus, Ann. Sci. École Norm. Sup. (4) 21 (1988), no. 3, 307-331. MR 974408 (89k:60087)

13. Gérard Ben Arous, Shigeo Kusuoka, and Daniel W. Stroock, The Poisson kernel for certain degenerate elliptic operators, J. Funct. Anal. 56 (1984), no. 2, 171-209. MR 738578 (85k:35093)

14. Jean-Michel Bismut, Martingales, the Malliavin calculus and hypoellipticity under general Hörmander's conditions, Z. Wahrsch. Verw. Gebiete 56 (1981), no. 4, 469-505. MR 621660 (82k:60134) 
15. Ugo Boscain, Robert Neel, and Luca Rizzi, Intrinsic random walks and sub-Laplacians in sub-Riemannian geometry, ArXiv e-prints (2015).

16. Roger W. Brockett, Lie algebras and Lie groups in control theory., Geom. Methods Syst. Theory, Proc. NATO advanced Study Inst., London, 43-82 (1973), 1973.

17. Bruce K. Driver and Tai Melcher, Hypoelliptic heat kernel inequalities on the Heisenberg group, J. Funct. Anal. 221 (2005), no. 2, 340-365. MR 2124868 (2005k:58044)

18. David Elworthy, Geometric aspects of diffusions on manifolds, École d'Été de Probabilités de Saint-Flour XV-XVII, 1985-87, Lecture Notes in Math., vol. 1362, Springer, Berlin, 1988, pp. 277-425. MR 983375 (90c:58187)

19. Michel Émery, Stochastic calculus in manifolds, Universitext, Springer-Verlag, Berlin, 1989, With an appendix by P.-A. Meyer. MR 1030543 (90k:58244)

20. Elisha Falbel, Claudio Gorodski, and Michel Rumin, Holonomy of sub-Riemannian manifolds, Internat. J. Math. 8 (1997), no. 3, 317-344. MR 1454476 (98e:53032)

21. Erlend Grong and Anton Thalmaier, Stochastic completeness and gradient representations for sub-Riemannian manifolds, Preprint arXiv:1605.00785.

22. __ Curvature-dimension inequalities on sub-Riemannian manifolds obtained from Riemannian foliations: part I, Math. Z. 282 (2016), no. 1-2, 99-130. MR 3448376

23. _ Curvature-dimension inequalities on sub-Riemannian manifolds obtained from Riemannian foliations: part II, Math. Z. 282 (2016), no. 1-2, 131-164. MR 3448377

24. Wolfgang Hackenbroch and Anton Thalmaier, Stochastische Analysis, Mathematische Leitfäden. [Mathematical Textbooks], B. G. Teubner, Stuttgart, 1994, Eine Einführung in die Theorie der stetigen Semimartingale. [An introduction to the theory of continuous semimartingales]. MR 1312827 (96e:60094)

25. Martin Hairer, On Malliavin's proof of Hörmander's theorem, Bull. Sci. Math. 135 (2011), no. 6-7, 650-666. MR 2838095 (2012m:60119)

26. Lars Hörmander, Hypoelliptic second order differential equations, Acta Math. 119 (1967), 147-171. MR 0222474 (36 \#5526)

27. Elton P. Hsu, Stochastic analysis on manifolds, Graduate Studies in Mathematics, vol. 38, American Mathematical Society, Providence, RI, 2002. MR 1882015 (2003c:58026)

28. Kanji Ichihara and Hiroshi Kunita, A classification of the second order degenerate elliptic operators and its probabilistic characterization, Z. Wahrscheinlichkeitstheorie und Verw. Gebiete 30 (1974), 235-254. MR 0381007 (52 \#1904)

29. - Supplements and corrections to the paper: "A classification of the second order degenerate elliptic operators and its probabilistic characterization" (Z. Wahrscheinlichkeitstheorie und Verw. Gebiete 30 (1974), 235-254), Z. Wahrscheinlichkeitstheorie und Verw. Gebiete 39 (1977), no. 1, 81-84. MR 0488328 (58 \#7877)

30. Nobuyuki Ikeda and Shinzo Watanabe, Stochastic differential equations and diffusion processes, second ed., North-Holland Mathematical Library, vol. 24, North-Holland Publishing Co., Amsterdam; Kodansha, Ltd., Tokyo, 1989. MR 1011252 (90m:60069)

31. Velimir Jurdjevic, Geometric control theory, Cambridge Studies in Advanced Mathematics, vol. 52, Cambridge University Press, Cambridge, 1997. MR 1425878 (98a:93002)

32. Shoshichi Kobayashi and Katsumi Nomizu, Foundations of differential geometry. Vol. I and II, Wiley Classics Library, John Wiley \& Sons, Inc., New York, 1996, Reprint of the 1963/1969 original, A Wiley-Interscience Publication. MR 1393940 (97c:53001a)/1393941 (97c:53001b)

33. Hiroshi Kunita, Supports of diffusion processes and controllability problems, Proceedings of the International Symposium on Stochastic Differential Equations (Res. Inst. Math. Sci., Kyoto Univ., Kyoto, 1976), Wiley, New York-Chichester-Brisbane, 1978, pp. 163-185. MR 536011 (80i:60108)

34. - On the decomposition of solutions of stochastic differential equations, Stochastic integrals (Proc. Sympos., Univ. Durham, Durham, 1980), Lecture Notes in Math., vol. 851, Springer, Berlin-New York, 1981, pp. 213-255. MR 620992 (83h:60065)

35. _ Stochastic differential equations and stochastic flows of diffeomorphisms, École d'été de probabilités de Saint-Flour, XII-1982, Lecture Notes in Math., vol. 1097, Springer, Berlin, 1984, pp. 143-303. MR 876080 (87m:60127)

36. _ Stochastic flows and stochastic differential equations, Cambridge Studies in Advanced Mathematics, vol. 24, Cambridge University Press, Cambridge, 1990. MR 1070361 (91m:60107)

37. Shigeo Kusuoka, Malliavin calculus revisited, J. Math. Sci. Univ. Tokyo 10 (2003), no. 2, 261-277. MR 1987133 (2004e:60089) 
38. Shigeo Kusuoka and Daniel Stroock, Applications of the Malliavin calculus. I, Stochastic analysis (Katata/Kyoto, 1982), North-Holland Math. Library, vol. 32, North-Holland, Amsterdam, 1984, pp. 271-306. MR 780762 (86k:60100a)

39. , Applications of the Malliavin calculus. II, J. Fac. Sci. Univ. Tokyo Sect. IA Math. 32 (1985), no. 1, 1-76. MR 783181 (86k:60100b)

40. - Applications of the Malliavin calculus. III, J. Fac. Sci. Univ. Tokyo Sect. IA Math. 34 (1987), no. 2, 391-442. MR 914028 (89c:60093)

41. Rémi Léandre, Majoration en temps petit de la densité d'une diffusion dégénérée, Probab. Theory Related Fields 74 (1987), no. 2, 289-294. MR 871256 (88c:60144)

42. Minoration en temps petit de la densité d'une diffusion dégénérée, J. Funct. Anal. 74 (1987), no. 2, 399-414. MR 904825 (88k:60147)

43. Hong-Quan Li, Estimation optimale du gradient du semi-groupe de la chaleur sur le groupe de Heisenberg, J. Funct. Anal. 236 (2006), no. 2, 369-394. MR 2240167 (2007d:58045)

44. Paul Malliavin, $C^{k}$-hypoellipticity with degeneracy, Stochastic analysis (Proc. Internat. Conf., Northwestern Univ., Evanston, Ill., 1978), Academic Press, New York-London, 1978, pp. 199214. MR 517243 (80i:58045a)

45. _,$C^{k}$-hypoellipticity with degeneracy. II, Stochastic analysis (Proc. Internat. Conf., Northwestern Univ., Evanston, Ill., 1978), Academic Press, New York-London, 1978, pp. 327340. MR 517250 (80i:58045b)

46. - Stochastic calculus of variation and hypoelliptic operators, Proceedings of the International Symposium on Stochastic Differential Equations (Res. Inst. Math. Sci., Kyoto Univ., Kyoto, 1976), Wiley, New York-Chichester-Brisbane, 1978, pp. 195-263. MR 536013 (81f:60083)

47. _ Stochastic analysis, Grundlehren der Mathematischen Wissenschaften [Fundamental Principles of Mathematical Sciences], vol. 313, Springer-Verlag, Berlin, 1997. MR 1450093 (99b:60073)

48. Tai Melcher, Hypoelliptic heat kernel inequalities on Lie groups, Stochastic Process. Appl. 118 (2008), no. 3, 368-388. MR 2389050 (2009a:58053)

49. James Norris, Simplified Malliavin calculus, Séminaire de Probabilités, XX, 1984/85, Lecture Notes in Math., vol. 1204, Springer, Berlin, 1986, pp. 101-130. MR 942019 (89f:60058)

50. David Nualart, The Malliavin calculus and related topics, second ed., Probability and its Applications (New York), Springer-Verlag, Berlin, 2006. MR 2200233 (2006j:60004)

51. Philip E. Protter, Stochastic integration and differential equations, Stochastic Modelling and Applied Probability, vol. 21, Springer-Verlag, Berlin, 2005, Second edition. Version 2.1, Corrected third printing. MR 2273672 (2008e:60001)

52. Daniel Revuz and Marc Yor, Continuous martingales and Brownian motion, third ed., Grundlehren der Mathematischen Wissenschaften [Fundamental Principles of Mathematical Sciences], vol. 293, Springer-Verlag, Berlin, 1999. MR 1725357 (2000h:60050)

53. Ichiro Shigekawa, On stochastic horizontal lifts, Z. Wahrsch. Verw. Gebiete 59 (1982), no. 2, 211-221. MR 650613 (83i:58102)

54. Daniel W. Stroock and S. R. S. Varadhan, On degenerate elliptic-parabolic operators of second order and their associated diffusions, Comm. Pure Appl. Math. 25 (1972), 651-713. MR 0387812 (52 \#8651)

55. - On the support of diffusion processes with applications to the strong maximum principle, Proceedings of the Sixth Berkeley Symposium on Mathematical Statistics and Probability (Univ. California, Berkeley, Calif., 1970/1971), Vol. III: Probability theory, Univ. California Press, Berkeley, Calif., 1972, pp. 333-359. MR 0400425 (53 \#4259)

56. Héctor J. Sussmann and Velimir Jurdjevic, Controllability of nonlinear systems, J. Differential Equations 12 (1972), 95-116. MR 0338882 (49 \#3646)

57. Philippe Tondeur, Foliations on Riemannian manifolds, Universitext, Springer-Verlag, New York, 1988. MR 934020 (89e:53052)

58. Shinzo Watanabe, Analysis of Wiener functionals (Malliavin calculus) and its applications to heat kernels, Ann. Probab. 15 (1987), no. 1, 1-39. MR 877589 (88h:60111)

59. David Williams, Probability with martingales, Cambridge Mathematical Textbooks, Cambridge University Press, Cambridge, 1991. MR 1155402 (93d:60002)

Mathematics Research Unit, FStC, University of Luxembourg, 6 Rue Richard CoudenhoveKalergi, L-1359 Luxembourg, Grand-Duchy of Luxembourg

E-mail address: anton.thalmaier@uni.lu 